\title{
Study of SNARE-mediated membrane fusion with a novel single vesicle fusion assay
}

\author{
Dissertation \\ for the award of the degree \\ "Doctor rerum naturalium" \\ of the Georg-August-Universität Göttingen \\ within the doctoral program Molecular Biology \\ of the Georg-August University School of Science (GAUSS)
}

\author{
submitted by \\ Agata Witkowska \\ from \\ Łódź, Poland
}

Göttingen, 2016 



\section{Thesis Advisory Committee:}

\section{Prof. Dr. Reinhard Jahn}

Department of Neurobiology

Max Planck Institute for Biophysical Chemistry

Prof. Dr. Andreas Janshoff

Institute of Physical Chemistry

University of Göttingen

Prof. Dr. Stefan Jakobs

Department of NanoBiophotonics

Max Planck Institute for Biophysical Chemistry

Department of Neurology

University Medical Center Göttingen

\section{Extended Examination Committee (alphabetically):}

Prof. Dr. Nils Brose

Department of Molecular Neurobiology

Max Planck Institute for Experimental Medicine

Prof. Dr. Tobias Moser

Institute for Auditory Neuroscience and InnerEarLab

University Medical Center Göttingen

Prof. Dr. Blanche Schwappach

Department of Molecular Biology

University Medical Center Göttingen

Oral examination: 23 November 2016 

Herewith I declare, that I prepared this Doctoral Thesis, entitled "Study of SNAREmediated membrane fusion with a novel single vesicle fusion assay", on my own and with no other sources and aids than quoted.

Göttingen, 30 September 2016 Agata Witkowska 



\section{Table of Contents}

$\begin{array}{ll}\text { Acknowledgements } & 11\end{array}$

$\begin{array}{ll}\text { Abstract } & 13\end{array}$

$\begin{array}{lll}1 & \text { Introduction } & 15\end{array}$

1.1 Chemical neurotransmission . . . . . . . . . . . . . . . . . 15

1.2 SNARE proteins in neuronal exocytosis . . . . . . . . . . . . . 17

1.2.1 Brief overview on the history of membrane fusion research . . . 17

1.2 .2 SNARE proteins . . . . . . . . . . . . . 18

1.2.3 Molecular details of synaptic vesicle fusion . . . . . . . . . . 20

1.2.4 Mechanics of SNARE-induced membrane fusion . . . . . . . . 22

1.3 Fusion assays . . . . . . . . . . . . . . . . . . . . . . . . . . . . 24

1.3.1 In vivo approaches to study neuronal exocytosis . . . . . . . . . 24

1.3.2 In vitro approaches to study neuronal exocytosis . . . . . . . . 25

1.4 Aims of this study . . . . . . . . . . . . . . . . . . . . . . 29

2 Materials and Methods 31

2.1 Materials . . . . . . . . . . . . . . . . . . . . . 31

2.2 Protein handling f . . . . . . . . . . . . . . . . . . . . . . . . . . . . . 31

2.2.1 Basic methods . . . . . . . . . . . . . . . . . . 31

2.2 .2 Protein labelling _. . . . . . . . . . . . . . . . . . . 34

2.2.3 SNARE acceptor complex formation . . . . . . . . . 35

2.3 Lipid vesicle preparation . . . . . . . . . . . . . . . . . . . 35 
2.3.1 Small unilamellar vesicles . . . . . . . . . . . . . . . . 35

2.3.2 Large unilamellar vesicles . . . . . . . . . . . . . 36

2.3.3 Giant unilamellar vesicles . . . . . . . . . . . . . . . . 37

2.3.4 Chromaffin granule purification and labelling . . . . . . . . . . 40

2.4 Bulk fluorescence anisotropy assay used for monitoring of the ternary SNARE complex formation . . . . . . . . . . . . . . . . . 42

2.5 Microscopy: imaging and analysis . . . . . . . . . . . . . 43

2.5.1 Fluorescence imaging . . . . . . . . . . . . . . . . . . . 43

2.5.2 The iSCAT microscopy . . . . . . . . . . . . . . 43

2.5.3 Immobilisation of GUVs . . . . . . . . . . . . . . . . . 44

2.5.4 Design of the GUV-based fusion assay . . . . . . . . . . . . 45

2.5.5 Basic analysis of microscopy data . . . . . . . . . . . . . 46

2.5.6 GUV reconstitution efficiency determination . . . . . . . . . 47

2.5.7 Diffusion coefficient determination of molecules incorporated into GUV membrane . . . . . . . . . . . . . . . . . . . . . . . 48

2.5.8 Detection of fusion by lipid mixing . . . . . . . . . . . . . 49

2.5.9 Detection of fusion by content mixing . . . . . . . . . . . . 51

2.5.10 Tracking of docked vesicles . . . . . . . . . . . . . 51

$\begin{array}{lll}3 & \text { Results } & 55\end{array}$

3.1 Optimisation and characterisation of $\Delta \mathrm{N}-\mathrm{GUVs} \ldots \ldots . \ldots 5$

3.1.1 Protocol development of $\Delta \mathrm{N}-\mathrm{GUV}$ formation . . . . . . . . 55

3.1.2 Morphological and biochemical characterisation of $\Delta \mathrm{N}-\mathrm{GUVs}$. . 59

3.1.3 Assessment of fusogenic activity of $\Delta \mathrm{N}-\mathrm{GUVs} \ldots \ldots 2$ 
3.2 SNARE-mediated docking and fusion of single vesicles to $\Delta$ N-GUVs . . 67

3.2.1 Docking of $\Delta 84$ syb-LUVs on GUV surface . . . . . . . . . 67

3.2.2 Single liposome fusion to GUVs . . . . . . . . . . . . . . . . . 69

3.2.3 Fusion of purified secretory granules to GUVs . . . . . . . . 71

3.3 Investigation of SNARE-mediated vesicle docking $\ldots \ldots \ldots$. . . . . 73

3.3.1 Docking and undocking . . . . . . . . . . . . . 73

3.3.2 Characterisation of mobility of docked vesicles . . . . . . . . 75

4 Discussion

4.1 Effect of the preparation method on the GUV quality . . . . . . . . . . 81

4.2 A novel platform for monitoring single vesicle fusion with high temporal and spatial resolution . . . . . . . . . . . . . . . . . . 84

4.3 The effect of SNARE complex assembly on vesicle docking and membrane fusion . . . . . . . . . . . . . . . . . . . . . . . . . 89

4.3.1 Reversibility of the SNARE complex assembly _ . . . . . . 89

4.3.2 Do two vesicle docking modes detected with iSCAT represent intermediates of membrane fusion pathway? . . . . . . . . . . 91

4.4 Conclusions and Outlook . . . . . . . . . . . . . . . . . . . . . . 94

$\begin{array}{ll}\text { Bibliography } & 97\end{array}$

$\begin{array}{ll}\text { Appendix } & 119\end{array}$

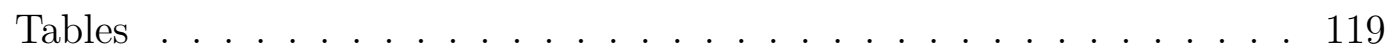

Scripts . . . . . . . . . . . . . . . . 126

List of Abbreviations

133 
List of Figures

135

List of Tables

List of Scripts

141

Curriculum vitae

143 


\section{Acknowledgements}

I would like to thank Professor Reinhard Jahn for mentoring and supervision during my $\mathrm{PhD}$ studies, and giving me the opportunity to work in his group. It was a real honour to work with you and share the passion for science.

I also would like to thank my teachers from the lab - Dr. Geert van den Bogaart (my lab rotation supervisor) and Dr. Halenur Yavuz. Thank you Geert for encouraging me to join the lab for my PhD. And, thank you Hale for your advice and friendship.

I am really grateful to members of my Thesis Advisory Committee - Professor Andreas Janshoff and Professor Stefan Jakobs - for their input into my project and discussions.

I would also like to acknowledge Professor Erwin Neher and Professor Mary Osborn for their support, career advice, and being scientist role-models.

I would like to thank all my present and past colleagues from the group, especially: Dr. Angel Perez-Lara - for scientific discussions and critical reading of parts of this work, and Dr. Yongsoo Park - for introducing me to chromaffin granule purification. Also, I would like to thank Dagmar Diezmann for sharing the lab space with me for all these years, and encouraging me to speak German.

I would like to thank my collaborators from Erlangen - Professor Vahid Sandoghdar and Susann Spindler. It is a pleasure to collaborate with you and work with such a cutting-edge technology.

My PhD would not be possible without the family of the Molecular Biology Program, especially Dr. Steffen Burkhardt and Kerstin Grüniger. Thank you!

Finally, I would like to thank my friends and family: my friend Tanvi — for support, especially during the first year of my stay in Germany; my Parents Dorota and Krzysztof - for infecting me with enthusiasm for academic life, and their constant support and love; and my husband Eukasz - for everything, especially sharing with me the life full of love and passion for science. 



\section{Abstract}

Membrane fusion in the secretory pathway of eukaryotic cells, including exocytosis of synaptic vesicles, is catalysed by SNARE proteins. In case of the synaptic vesicle fusion, two of the members of this protein family reside on the presynaptic plasma membrane and one on synaptic vesicles. When these three proteins come together they undergo an exergonic reaction of zippering, from membrane distal to membrane proximal regions, to form a coiled coil structure. This process pulls membranes towards each other and induces membrane fusion. Despite many years of research, the molecular mechanism of the SNARE-driven fusion is still not fully understood, and remaining questions concern the extraordinary synaptic vesicle exocytosis speed, or translation of the zippering force to fusing membranes.

In vitro, SNAREs are sufficient to mediate effective fusion of both native and artificial membranes. Over the past years, reconstitution systems have been instrumental in characterising the basic features of the SNARE-mediated membrane fusion in combination with various accessory proteins (especially these involved in synaptic vesicle exocytosis). First, ensemble measurements of SNARE-mediated liposome fusion were performed, however they lacked the possibility of distinguishing of reaction steps. Therefore, more recently, microscopy assays were developed that allow observation of the fusion reaction on a single vesicle level. Nevertheless, these assays often lack temporal resolution for monitoring fast fusion reaction catalysed by neuronal SNAREs, and usually involve membrane immobilisation to a solid surface that may lead to various artefacts.

In this study a novel single vesicle assay was developed for monitoring membrane fusion on the example of neuronal exocytosis. This assay includes giant unilamellar vesicles as presynaptic plasma membrane mimics and smaller vesicles (either liposomes or purified secretory granules). Membrane topology of giant liposomes ensures that only a small portion of the membrane is involved in interactions with the surface. Additionally, this free-standing membrane is largely free of curvature stress, similarly as presynaptic plasma membrane. Assay allows monitoring ms-kinetics of secretory vesicle fusion, that is close to fusion rates observed in vivo in neuroendocrine cells. What is more, due to correct membrane topology, docked vesicles are very mobile and the mechanism of docking can be studied in great detail. The assay presented in this study is also very versatile as it can be adapted for other studies concerning for example endosomal membrane fusion or viral cell entry. 



\section{Introduction}

\subsection{Chemical neurotransmission}

The basis of the chemical neurotransmission were described by Katz and colleagues in the 1960s (Katz, 1969) based on their work on the neuromuscular junction (selected references: del Castillo and Katz, 1954 - quantal release theory; Katz and Miledi, 1967 - release triggering by calcium) and previous studies on vesicle trafficking by George Palade (Palay and Palade, 1955). The chemical neurotransmission occurs when an action potential arrives at a presynaptic nerve terminal, gates the $\mathrm{Ca}^{2+}$ channels (voltage-gated), and the $\mathrm{Ca}^{2+}$ influx triggers exocytosis of synaptic vesicles containing neurotransmitter that diffuses into the synaptic cleft and binds to the neurotransmitter receptors on the membrane of a postsynaptic cell (Figure 1.1) eliciting a postsynaptic potential. Despite many years of research, the full understanding of this process is still missing. The basic questions posed by the initial work in the 1960s are still open (Südhof, 2013), namely:

1. How do vesicles fuse with the membrane? - This question is central not only in neurobiology but in all areas of cell biology dealing with vesicle traffic. The process of transporting cargo and membranes is universal for all eukaryotic cells (a classical review on this topic is written by Bonifacino and Glick, 2004). Details about the current understanding of the molecular mechanism of this process are presented in the following sections.

2. How does $\mathrm{Ca}^{2+}$ influx trigger exocytosis? - The $\mathrm{Ca}^{2+}$-triggered exocytosis is also not only limited to neurons but occurs in many different types of cells including for example endocrine (e.g. chromaffin cells in adrenal glands) and exocrine cells, or acrosome fusion in sperm cells (various types of $\mathrm{Ca}^{2+}$-triggered fusion are reviewed in Kasai et al., 2012). Currently, there is a consensus on what the "calcium sensors" of vesicles are, but the exact mechanism remains still a matter of debate.

3. How is sub-millisecond vesicle fusion kinetics achieved? - It was shown that in fast mammalian synapses release of the neurotransmitter can occur in microseconds after opening of $\mathrm{Ca}^{2+}$ channels (Sabatini and Regehr, 1996; also reviewed for example in Neher and Sakaba, 2008). This process requires (a) tight control over the spatial organisation of release sites (e.g. regarding the distance 
between the calcium influx site and the vesicle), as well as (b) highly efficient molecular machinery that would ensure membrane merger in such a short time.

(a) Spatial organisation of release sites at the presynaptic nerve terminal is ensured by a structure called active zone that was identified as an electron dense region at the neuromuscular junction by Couteaux and PécotDechavassine (1970). Nowadays, there is much more understanding about the molecular composition of these release sites (reviewed by Südhof, 2012), but the functional explanation is still missing.

(b) The molecular machinery specialised for performing the fast neuroexocytosis has been identified over the last two decades (reviewed in Jahn and Fasshauer, 2012; and described in the following sections). However, how these proteins perform their function is still unclear.

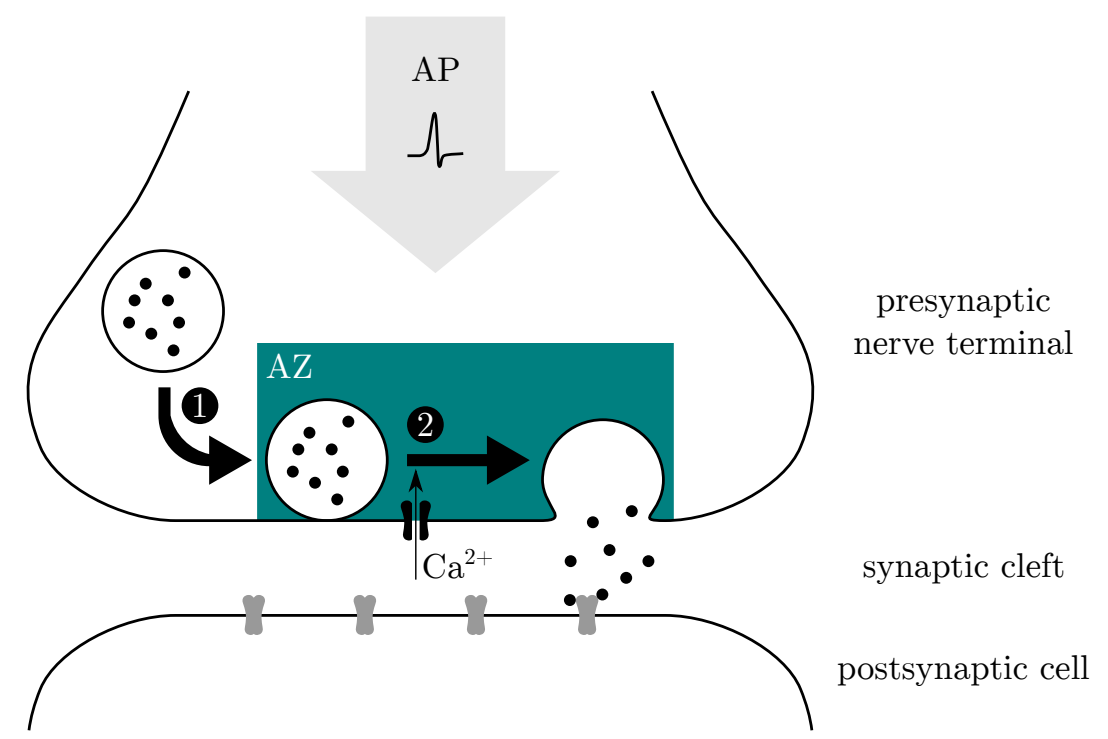

Figure 1.1. Chemical neurotransmission principle. Schematic diagram presenting the principle of the chemical neurotransmission. Synaptic vesicles filled with neurotransmitter dock to the presynaptic plasma membrane (1) at the active zone (AZ). Action potential (AP) triggers opening of the voltage-gated calcium channels. Calcium influx into the cell induces exocytosis of synaptic vesicles (2). Neurotransmitter is then released to the synaptic cleft and binds to receptors present on the postsynaptic cell. Based on Jahn and Fasshauer (2012). 


\subsection{SNARE proteins in neuronal exocytosis}

\subsubsection{Brief overview on the history of membrane fusion research}

The first proteins known to induce membrane fusion were viral fusion proteins including one of the best studied fusogens - the hemagglutinin glycoprotein of the influenza virus (reviewed in Wiley and Skehel, 1987; Blijleven et al., 2016). Three independent lines of research contributed to the understanding of the membrane fusion occurring during vesicular trafficking in eukaryotic cells (Jahn, 2008): (i) genetic screens that enabled identification of proteins involved in the secretory pathway, (ii) cell-free assays that enabled biochemical analysis, and (iii) the structural and biophysical characterisation of proteins from trafficking organelles (mostly synaptic vesicles).

The first genetic screen that allowed identification of proteins involved in secretory pathway was initially intended for description of mutants with defects in the nervous system and was carried out in the nematode Caenorhabditis elegans by Sydney Brenner (1974). Many of the "uncoordinated" mutants identified in this study turned out to have a defect in the neurotransmitter release machinery. Most notably the UNC-13 and UNC-18 (mammalian homologues are called Munc13 and Munc18) proteins that belong to one of the most important accessory proteins regulating the synaptic vesicle exocytosis.

The second genetic screen that was central to the development of the membrane fusion field was performed with Saccharomyces cerevisiae by Novick and Schekman (Novick et al., 1980). In this and subsequent studies hundreds of genes involved in the secretory pathway were discovered, including SM (Sec1/Munc18-like) and Rab proteins that play a role in the preparation of membrane fusion (Sec1 and Sec4), SNARE proteins that catalyse membrane merger (Sec9 and Sec22), and NSF with SNAP protein that regenerate SNAREs after fusion (Sec18 and Sec17).

The biochemical characterisation of the fusion reaction was enabled by the development of the cell-free membrane fusion assays. The first experiments were done in permeabilised chromaffin cells (Baker and Knight, 1978) and sea urchin eggs (Vacquier, 1975) to characterise the $\mathrm{Ca}^{2+}$-triggered exocytosis. Subsequent study by Rothman and colleagues (Balch et al., 1984) has enabled the description of protein components involved in vesicle budding and fusion, thanks to a cell-free assay that demonstrated transport of a protein between the successive compartments of the Golgi apparatus. 
This work has led to the identification of the complex of neuronal SNARE proteins (syntaxin, SNAP-25, and synaptobrevin) implicated in membrane fusion, in experiments with the affinity purification from bovine brain using NSF (N-ethylmaleimide-sensitive factor) as a bait (Söllner et al., 1993b). Later the same year, the theory of SNARE complex assembly-disassembly pathway was formulated (Söllner et al., 1993a). According to this initial theory, SNAREs residing on the opposite fusing membranes (in this case it was known that synaptobrevin resides on synaptic vesicles) would form a complex and the disassembly process mediated by the NSF with the energy from ATP hydrolysis would trigger fusion.

Afterwards, another cell-free assay by Wickner and colleagues using fusion of yeast vacuoles (Mayer et al., 1996; Nichols et al., 1997) has proven that NSF and SNAPs are not required for the docking and fusion. Subsequently, in 1997 it was demonstrated that SNARE proteins in a complex are organised in a parallel way and it was proposed that the N- to C-terminal exergonic zippering of SNARE proteins residing on different membranes leads to fusion (Hanson et al., 1997; Lin and Scheller, 1997). This was then followed by a first crystal structure of a neuronal SNARE complex in 1998 (Sutton et al., 1998) presenting the soluble fraction of the proteins, completed by the structure by Stein et al. (2009) that included also transmembrane regions (see also Figure 1.2). Finally, experiments involving reconstitution of purified recombinant SNARE proteins on liposomes have proven that SNAREs constitute the core fusion machinery and do not need any cofactors to perform membrane fusion (Weber et al., 1998).

\subsubsection{SNARE proteins}

The SNARE (SNAP receptor) proteins are fusogens taking part in the vesicular trafficking pathways in eukaryotes (Kloepper et al., 2007). They are small and mostly membrane-anchored, and can be distinguished by a stretch of 60-70 amino acids arranged in heptad repeats, a so called SNARE motif (Sutton et al., 1998; Kloepper et al., 2007). During the SNARE complex formation, SNARE motifs in a zipper-like fashion assemble forming a coiled coil (Sutton et al., 1998). This structure is stabilised by 15 layers of hydrophobic interactions (Figure 1.2) and one ionic layer at the centre (layer zero) that usually consists of one arginine $-\mathrm{R}$ and three glutamines - $\mathrm{Qa}$, Qb, Qc (Sutton et al., 1998; Fasshauer et al., 1998). SNARE proteins, based on their residue at the layer zero and the position in the SNARE complex were divided into four groups: R-, Qa-, Qb-, and Qc-SNAREs (Fasshauer et al., 1998). For the SNARE 


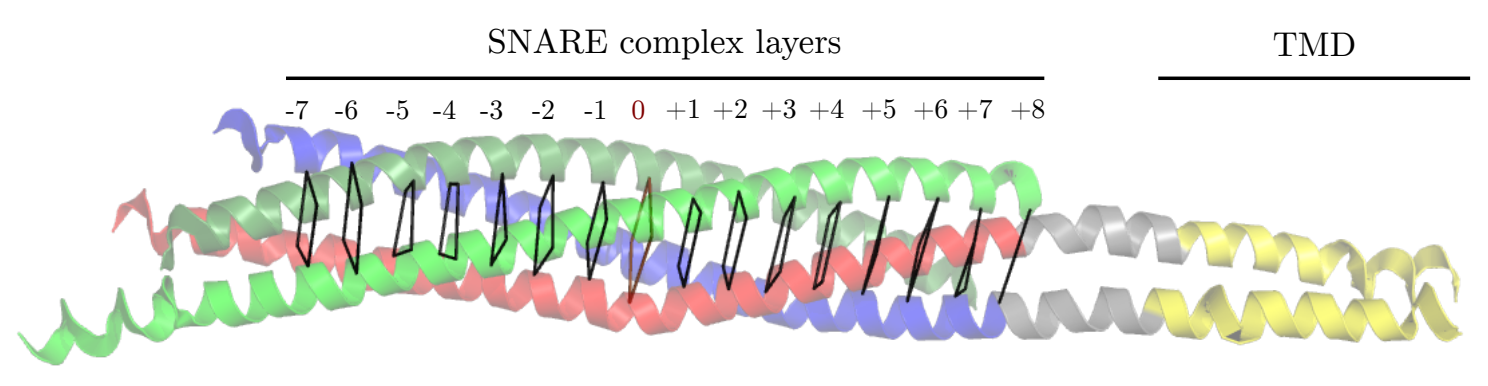

Figure 1.2. Neuronal SNARE complex structure. This structure (PDB ID: 3IPD; Stein et al., 2009) consists of SNARE motifs and additionally C-terminal transmembrane domains (TMD, labelled in yellow) of syntaxin and synaptobrevin. Syntaxin shown in red, SNAP-25 in green, and synaptobrevin in blue. Linkers between the SNARE motif and TMDs are indicated in grey. Between helices marked virtual bonds between $\mathrm{C} \alpha$ atoms interpreted as layers of the SNARE complex (Sutton et al., 1998; Fasshauer et al., 1998). The "zero" layer is indicated in brown. Figure prepared in PyMOL (Schrödinger, LLC), based on Hernandez et al. (2012).

complex formation, four SNARE motifs coming from four different SNARE protein groups are required (Figure 1.2).

SNARE proteins undergo assembly and disassembly cycles (Figure 1.3; Jahn and Scheller, 2006). The Qa, Qb, and Qc monomers are believed to form a so called acceptor complex anchored on the target membrane (Figure $1.3 \mathrm{~A}$ ). In the second step, after the arrival of a vesicle, the membrane anchored R-SNARE zippers with the Q-SNAREs in the direction from the membrane distal N-terminus to membrane proximal C-terminus and thereby pulls membranes tightly together (Figure 1.3 B-C). This energy releasing process is postulated to induce the fusion of two membranes and transition of the SNARE complexes from the trans-configuration (on two apposed membranes, Figure 1.3 C) to the cis-configuration (located on the same membrane, Figure $1.3 \mathrm{D})$. The cis-complexes are then disassembled to mostly unstructured monomers by NSF with the energy from ATP hydrolysis, and with $\alpha$ SNAP as a cofactor (Figure $1.3 \mathrm{E}-\mathrm{F})$.

SNARE complexes formed from recombinantly expressed neuronal SNAREs (lacking membrane attachment) are resistant to sodium dodecyl sulfate (SDS) and are not cleaved by neurotoxins that cleave monomers (Hayashi et al., 1994). These complexes have also high thermal stability in $70-90{ }^{\circ} \mathrm{C}$ (Fasshauer et al., 1997b).

As this work used SNARE proteins required for fast neuronal exocytosis for investigation of membrane fusion mechanism, further description will mainly concentrate on syntaxin-1A (Qa, contains transmembrane domain, further referred to as syntaxin), 


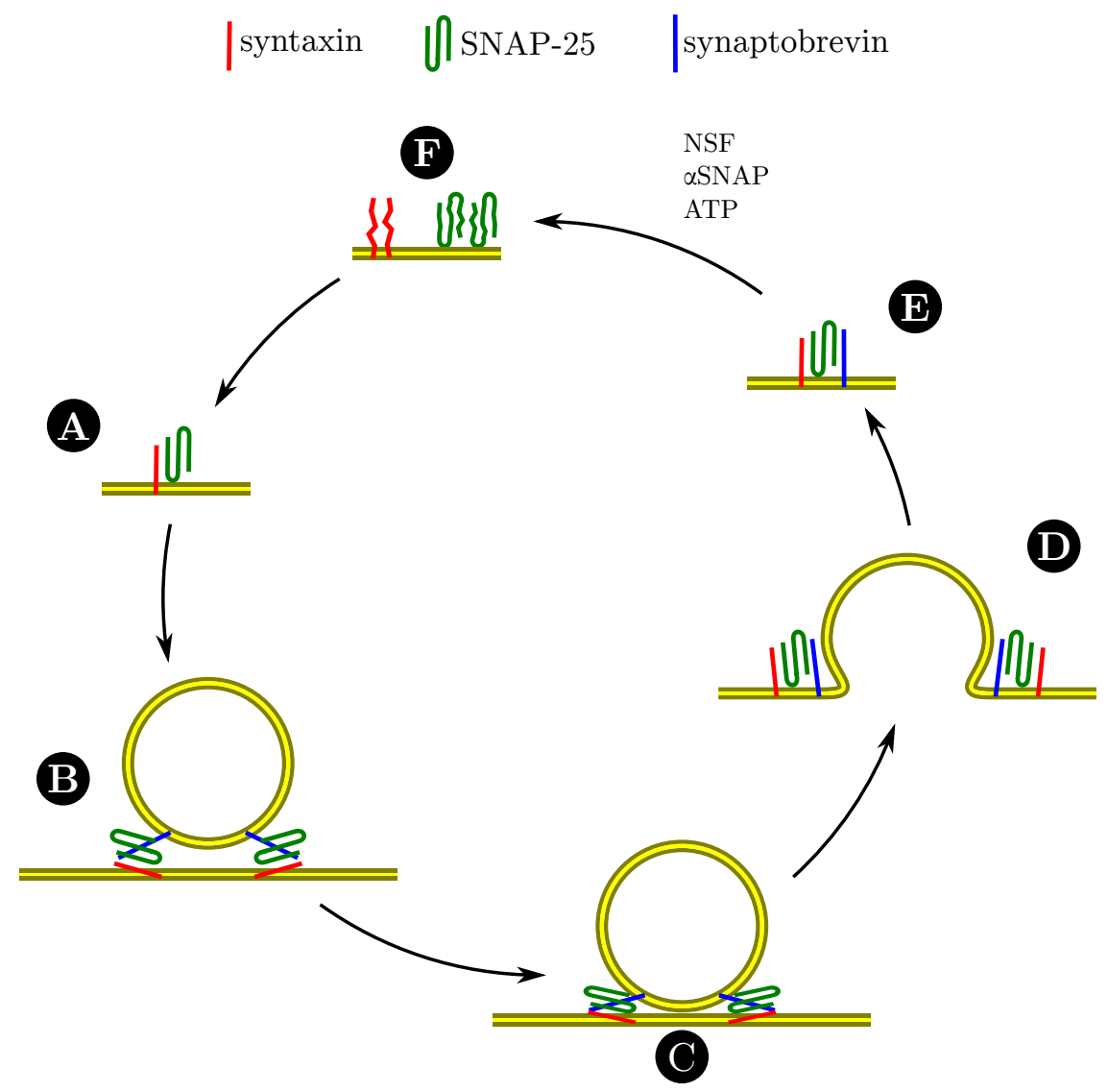

Figure 1.3. The SNARE cycle. Diagram presenting assembly-disassembly cycle of SNARE proteins on example of a neuronal SNARE complex. (A) SNARE acceptor complex is formed on the plasma membrane. Then, syb from synaptic vesicle forms a loose (B) and subsequently tight (C) SNARE complex with acceptor SNAREs, and thereby pulls the vesicle towards the plasma membrane. This results in membrane fusion (D). Next, cis-SNARE complex is disassembled (from E to F) by NSF with the energy from ATP and with its cofactor aSNAP. Based on: Jahn and Fasshauer (2012); Baker and Hughson (2016).

SNAP-25 (two SNARE motifs Qb and Qc, attached to the membrane by palmitoylation), and sybaptobrevin-2 (VAMP-2, an R-SNARE that contains a transmembrane domain, later referred to as syb) presented as a SNARE complex on Figure 1.2.

\subsubsection{Molecular details of synaptic vesicle fusion}

The synaptic vesicle exocytosis defines how much of the neurotransmitter is going to be released to the synaptic cleft during an action potential. During resting conditions synaptic vesicles are stored in nerve terminals. Some vesicles are attached to the presynaptic plasma membrane in the region of the active zone. Action potential initiates 
opening of the voltage-gated calcium channels leading to the calcium influx and in result the rate of exocytosis increases 100,000 fold (see: Figure 1.1; Jahn and Fasshauer, 2012).

Proteins mediating neuronal exocytosis belong to structurally conserved protein families like: SNAREs (mediate fusion), Rab proteins (important for tethering), SM proteins (Munc18), or CATCHR (complexes associated with tethering containing helical rods) proteins (Munc13; see also in the following text). There are also specialised groups of regulatory proteins responsible for the $\mathrm{Ca}^{2+}$-triggering, namely synaptotagmins ( $\mathrm{Ca}^{2+}$ sensors) and complexins.

Deletion of either Munc18 or Munc13 leads to complete exocytosis block (Verhage et al., 2000; Varoqueaux et al., 2002). Munc18 was initially found to interact with a closed conformation of syntaxin where the N-terminal accessory domain (Habc domain) binds to the SNARE motif inhibiting formation of a fusion complex (Dulubova et al., 1999; Misura et al., 2000). Later, the second binding mode of Munc18 and syntaxin was found involving syntaxin N-terminal peptide (Burkhardt et al., 2008). Munc13 is a large (around $200 \mathrm{kDa}$ ) active zone protein that was suggested to take part in opening the inhibitory Munc18-syntaxin complex (Richmond et al., 2001). Together, Munc18 and Munc13 probably guide SNARE proteins through the initial part of the assembly pathway (Ma et al., 2013; Baker et al., 2015; Baker and Hughson, 2016).

The $\mathrm{Ca}^{2+}$-dependent exocytosis requires synaptotagmins and complexins. Synaptotagmin-1 (later referred to as synaptotagmin) is a calcium-sensor required for the synchronous neurotransmitter release in neurons. It contains a transmembrane domain that anchors it to the synaptic vesicle membrane, and two $\mathrm{C} 2$ domains (C2A and $\mathrm{C} 2 \mathrm{~B}$ ) that bind $\mathrm{Ca}^{2+}$ ions. The $\mathrm{C} 2$ domains bind to membranes containing acidic phospholipids (phosphatidylserine - PS) in a $\mathrm{Ca}^{2+}$-dependent manner (Brose et al., 1992). Additionally, the C2B domain binds to PIP2 (phosphatidylinositol 4,5-bisphosphate) enriched plasma membrane (Bai et al., 2004). It is currently controversially discussed whether or not synaptotagmin binds to the SNARE complex (evidence for both theories presented in: Zhou et al., 2015; Park et al., 2015). On the other hand, complexins are known to bind the SNARE complex on the groove created by helices of syntaxin and synaptobrevin through its central helix in an antiparallel fashion (Bracher et al., 2002; Chen et al., 2002). Two alternative mechanisms are considered: complexin either promotes progression of zippering and sensitisation of SNAREs to the activation by synaptotagmin (e.g. Xue et al., 2010), or acts as a clamp that blocks SNARE complex in a partially zippered state until the $\mathrm{Ca}^{2+}$ signal arrives (e.g. Yang et al., 2010). 
In summary, in order to explain the synaptic vesicle exocytosis, currently two models (i and ii) are debated as discussed in Jahn and Fasshauer (2012). First, SNARE proteins are probably activated by active zone proteins, including Munc18 and Munc13. Then, either (i) a partially zippered SNARE complex is formed possibly with a clamp complexin, or (ii) vesicle is docked to the plasma membrane by other means e.g. synaptotagmin. Subsequently, upon $\mathrm{Ca}^{2+}$ influx, (i) complex is disinhibited and complexin is probably released, or (ii) synaptotagmin pulls the vesicle towards the plasma membrane and induces SNARE complex assembly. Assembly of the SNARE complex induces then merger of the synaptic vesicle and the plasma membrane, and release of the neurotransmitter through a fusion pore.

The following questions, actually relating back to the initial questions posed by the work of Bernard Katz in 1960s (Section 1.1), remain open regarding the molecular mechanism of the synaptic vesicle exocytosis:

1. What is the organisation of SNARE proteins prior to synaptic vesicle arrival at the fusion site? (the nature of acceptor complex, see also Discussion)

2. How are synaptic vesicles initially docked at the plasma membrane?

3. Is SNARE complex partially zippered before the $\mathrm{Ca}^{2+}$ influx? (see also Discussion)

4. How is the $\mathrm{Ca}^{2+}$-mediated triggering achieved by synaptotagmin and complexin?

5. How does the mechanical force created through SNARE complex zippering translate to merger of two apposed membranes? (discussed in the following section)

\subsubsection{Mechanics of SNARE-induced membrane fusion}

One of the most plausible explanations for the mechanism of membrane fusion is a stalk model developed by Kozlov and coworkers (Kozlov and Markin, 1983; Chernomordik and Kozlov, 2008), based on theory by Helfrich (1973) that treated membranes as elastic sheets. According to the stalk hypothesis (Figure 1.4), the fusion process proceeds through generation of a "point-like membrane protrusion" that later transforms to a stalk - a hourglass-shaped connection between two apposed monolayers. Further, two scenarios are considered - either the stalk can further expand to form the hemifusion diaphragm that transforms later to a fusion pore (steps 3a and 4 in Figure 1.4), or stalk transforms directly to a fusion pore (step 3b on Figure 1.4). 


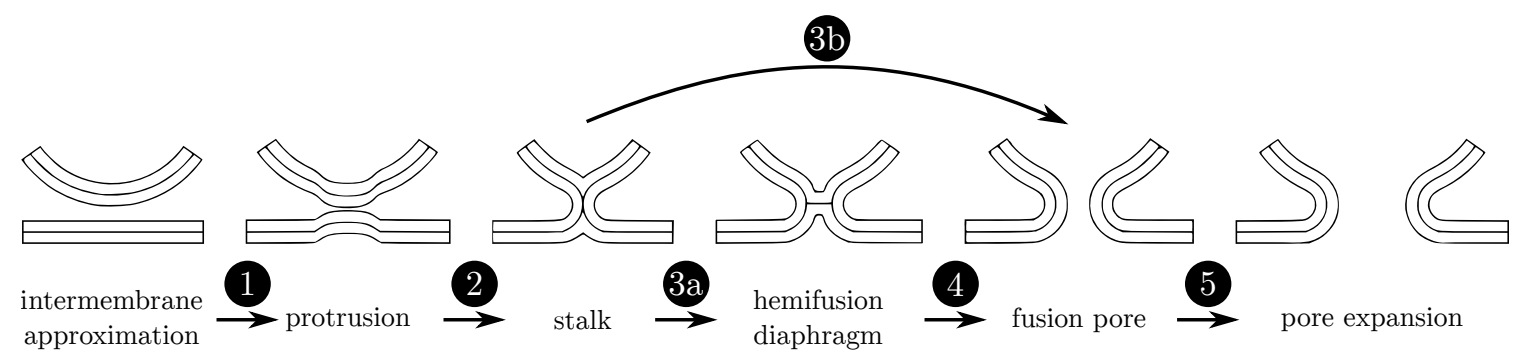

Figure 1.4. Hypothetical transition states in membrane fusion. Membrane fusion starts when two approximated lipid bilayers (shown here as separate monolayers) form a protrusion (1). In the next step stalk is formed (2) that can either expand to a hemifusion diaphragm (3a) or transition directly (3b) to a fusion pore formation (4). Finally, fusion pore dilates (5) to find energy minimum. Figure based on Rizo et al. (2006), modified according to Chernomordik and Kozlov (2008).

Membranes approaching each other have to overcome electrostatic repulsion forces that arise from the membrane surface (e.g. charged phospholipid headgroups). When bilayers are around $2 \mathrm{~nm}$ apart from each other, hydration forces, generated by the last layer of water molecules separating membranes, create a major energy barrier (Rand, 1981). Based mainly on molecular simulations, SNAREs are hypothesised not only to bring membranes into close proximity, but also actively guide fusing membranes by overcoming energy barriers up to the stage of the fusion pore expansion (reviewed in Risselada and Grubmüller, 2012). Based on simulations at near-atomic resolution (Risselada et al., 2011), SNAREs are believed to cause distortions in lipid packing of outer membrane leaflets and thereby induce the first lipid bridge formation by splaying of aliphatic tails.

Recently, thanks to electron microscopy techniques, initial intermediate states of SNARE-mediated membrane fusion were visualised (Hernandez et al., 2012). These intermediate states could be then correlated to the degree of SNARE complex zippering and ability to fuse liposomes of different diameter (Hernandez et al., 2012). One interesting SNARE mutation explored in this study was $\Delta 84 \mathrm{syb}$ (single amino acid deletion at +8 layer of the SNARE complex, see Figure 1.2) that was able to fuse small liposomes ( $\sim 40 \mathrm{~nm}$ in diameter) but not the large ones $(\sim 100 \mathrm{~nm}$ in diameter) that were stalled at the tightly docked state with apposed bilayers pressed against each other (Hernandez et al., 2012). In a more recent study (Yavuz, 2015) it turned out that this state cannot be reversed and is SNARE independent, suggesting a development of hydrophobic adhesion forces between the bilayers. The development of this tight docking intermediate is also possible with AA syb mutation laying upstream (Yavuz, 2015 ) in the layer -3 (I45A, M46A substitutions in syb) but takes longer (thus prob- 
ably requires higher energy from assembly of more SNARE complexes per one fusion site).

\section{$1.3 \quad$ Fusion assays}

For studying biological membrane fusion, generally two approaches can be used. In the first one, membrane fusion is measured in the conditions closest to the natural ones (e.g. in cells), by varying possibly least parameters of the system (in this work referred to as in vivo approaches). In contrast, in the second one the experimenter tries to build up the system form separate constituents i.e. lipids and proteins (in vitro approaches). Review over both types of approaches with emphasis on the process of the neuronal exocytosis is given in the following two sections.

\subsubsection{In vivo approaches to study neuronal exocytosis}

A lot of knowledge especially about regulatory steps of the synaptic vesicle exocytosis came from electrophysiological measurements in chromaffin cells and later in neurons. Neuroendocrine chromaffin cells were long used as neuronal cells models due to their neuro-ectodermal origin. Catecholamines are released from chromaffin cells in a process of $\mathrm{Ca}^{2+}$-triggered exocytosis of large granules, and this process resembles functionally and also biochemically the process of the synaptic vesicle exocytosis (exceptions are: the lack of the active zone and slower kinetics; reviewed for example in Stevens et al., 2011). Membrane fusion can be measured with electrophysiological methods indirectly by monitoring the release of the neurotransmitter with amperometry (Leszczyszyn et al., 1990; Chow et al., 1992), or even more indirectly by the measurement of neurotransmitter-triggered postsynaptic potentials. The direct measurement of membrane fusion is possible when cell membrane surface change due to the exocytosis is monitored with a capacitance measurement (Neher and Marty, 1982). These methods give basic information about the neurotransmitter release and the exocytosis timing, but lack the information about the steps preceding exocytosis, except for indirect evidence coming from cells lacking proteins for example involved in the preparation of a fusion machinery (e.g. UNC-18 mutants discussed in Section 1.2.1).

Another approach that can be used in in vivo context is imaging. The first snapshots of synaptic vesicle exocytosis were obtained by coupling electrical stimulation with electron microscopy (Heuser et al., 1979; Heuser and Reese, 1981). However, for 
the full understanding of the synaptic vesicle fusion, approaches allowing observation of dynamics of this process were needed. For this purpose, light microscopy techniques (especially total internal reflection fluorescence - TIRF microscopy) were instrumental, since there diffusion, docking, and fusion could be observed in the same preparation on a single vesicle level. In this method vesicles have to be labelled and usually it was achieved by either labelling them with a fluid phase marker that would get incorporated into vesicles during recycling rounds (e.g. with amphiphilic styryl dye FM 1-43 - Betz and Bewick, 1992), or by genetically encoded fluorescent markers (e.g. synaptopHluorin - Miesenböck et al., 1998). In this way, exocytosis could be visualised in chromaffin cells (Steyer et al., 1997), at the ribbon-type synapses (Zenisek et al., 2000), or more recently in a fast nerve terminal from the central nervous system (Midorikawa and Sakaba, 2015). Currently, more elegant systems are being developed that would allow even better imaging of the synaptic vesicle exocytosis (for example so called "xenapses" — Nosov et al., 2016).

The last group of in vivo approaches is observation of vesicle fusion in altered cells. First, there were experiments with permeabilised cells (described in Section 1.2.1). Another type of a fusion assay that was developed (Avery et al., 2000) involved membrane patches generated by sonication of PC12 cells (neuroendocrine cell line; Greene and Tischler, 1976) that had still secretory granules attached to the plasma membrane. In this way experimenter gained access to manipulation of intracellular factors, for example soluble proteins form the cytosol (Avery et al., 2000), or could alter the cytoplasmic membrane leaflet composition (Lang et al., 2001).

\subsubsection{In vitro approaches to study neuronal exocytosis}

The first evidence that SNAREs constitute the main catalysts of membrane fusion came from experiments where purified proteins were reconstituted onto liposomes (Weber et al., 1998). In this study (Weber et al., 1998), two sets of liposomes had reconstituted either plasma membrane SNAREs or a synaptic vesicle SNARE (syb), and upon mixing of these two types of vesicles membrane fusion would occur. A well established lipid mixing assay (Struck et al., 1981) was used to monitor the reaction where one set of vesicles contained NBD-PE and rhodamine-PE that were exhibiting fluorescence resonance energy transfer (FRET). Upon fusion fluorescently labelled lipids would get diluted with unlabelled lipids coming from another vesicle, and thus the donor dequenching can be observed. Since then, so called fusion assays were adapted in many different ways. 
One of the adaptations made in order to study the effect of membrane curvature on fusion kinetics was the use of larger liposomes, namely large unilamellar vesicles (LUVs, $\sim 100 \mathrm{~nm}$ in diameter; Hernandez et al., 2012), or giant unilamellar vesicles (GUVs, $>1$ um; Malsam et al., 2012), instead of small unilamellar vesicles (SUVs, $40 \mathrm{~nm}$; Weber et al., 1998). Then, also a possibility to monitor hemifusion was introduced with a system that would quench the outer leaflet of liposome membrane bilayer with dithionite based on the method developed by McIntyre and Sleight (1991; SNAREmediated fusion - Hernandez et al., 2012). Another modification was to monitor SNARE complex formation leading to membrane fusion by FRET measurements involving fluorophores attached to luminal (C-terminal) parts of syntaxin and synaptobrevin (Schuette et al., 2004). Finally, assays that would monitor successful pore formation were proposed (so called "content mixing"; primary assay ideas: Ingolia and Koshland, 1978; Wilschut et al., 1980; Niles and Cohen, 1987; SNARE-mediated fusion: Nickel et al., 1999; Hu et al., 2003; Bowen et al., 2004; Diao et al., 2010; Kyoung et al., 2011). For more details see Section 2.5.8 and 2.5.9.

Most of the described above assays can be classified as so called "bulk fusion assays" that are characterised by ensemble measurements of the reaction progress. Although bulk fusion assays have been instrumental in unrevealing some details of the SNAREmediated fusion, they do not allow to distinguish discrete steps of the reactions like docking and fusion. In order to access information about steps preceding fusion, single vesicle approaches were needed. Currently, there is a number of assays allowing observation of fusion events on a single vesicle level (Figure 1.5).

The first assay that allowed observation of single vesicle SNARE-mediated fusion was presented by Fix et al. (2004), and later the same year Bowen et al. (2004) also introduced monitoring of the content release. These assays involved supported lipid bilayers (SLBs) with reconstituted plasma membrane SNAREs (syntaxin and SNAP-25) and liposomes with syb. Already first measurements revealed that SNAREs can induce membrane fusion with much faster kinetics that previously inferred from bulk assays. However, these first assays had some problems like for example low number of mobile proteins in SLBs, or that fusion had to be induced by heating (Bowen et al., 2004). This assay was then further modified by adding polymer-conjugated lipids into the SLB that could potentially increase the space between the substrate (microscope coverslip) and bilayer, and improve protein mobility (Karatekin et al., 2010). Another variation of this method involved different method of SLB formation (Kalb et al., 1992) that required in the first step formation of a lipid monolayer (protein free) with a Langmuir-Blodgett technique (Blodgett, 1935; Blodgett and Langmuir, 1937) and 


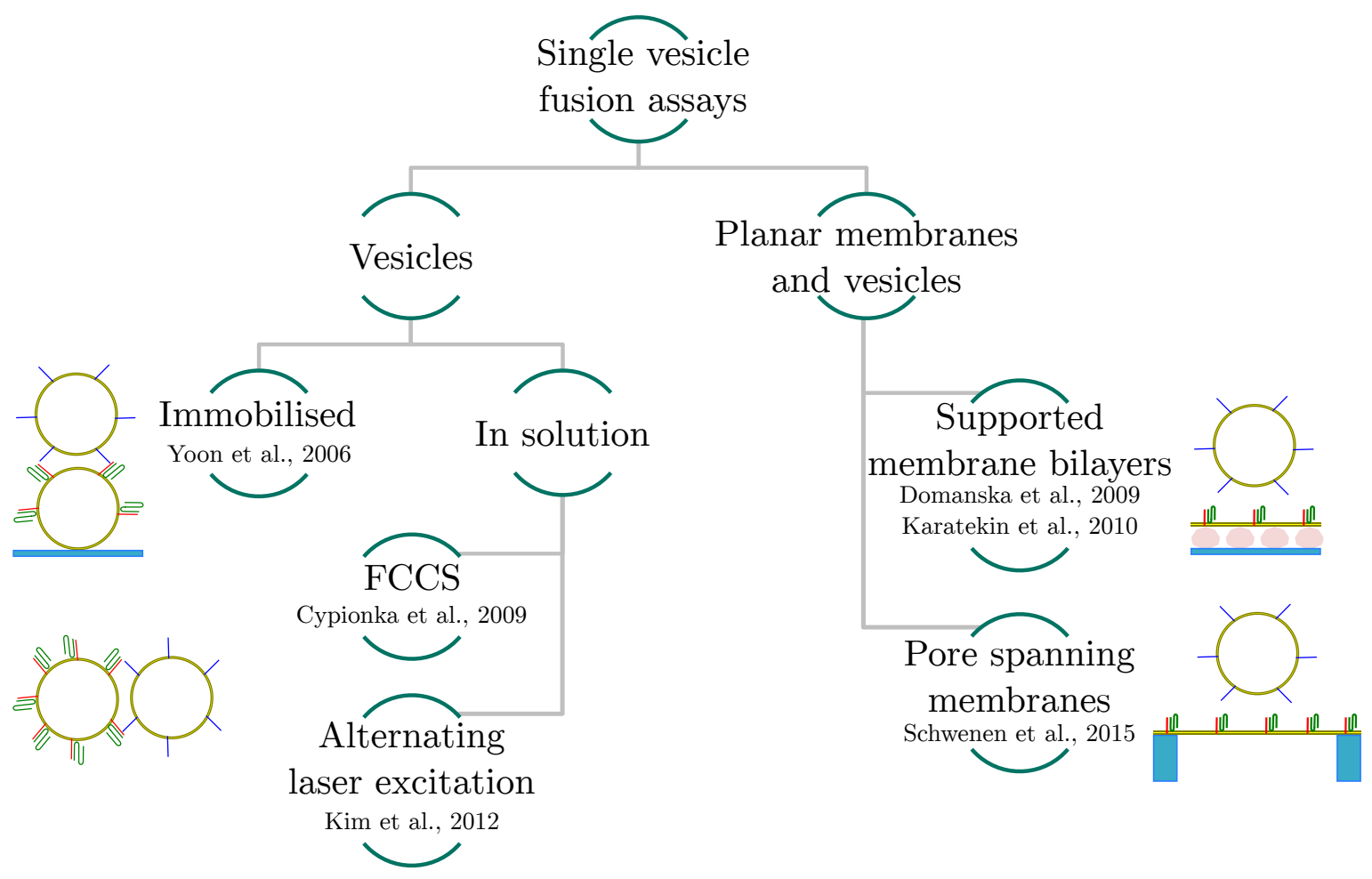

Figure 1.5. Single vesicle fusion assays. Diagram presenting an overview of single vesicle fusion assays studying SNARE-mediated fusion with representative references. Description in text.

subsequently, in the second step fusion of protein containing vesicles that would complete the membrane bilayer. In this way, asymmetry in leaflet composition can be maintained (Crane et al., 2005), and SNARE proteins are preferentially oriented towards the solution and not the substrate, and hence have also higher mobility (Wagner and Tamm, 2000, 2001). So far, with this assay modification, fastest SNARE-mediated fusion events were observed that took only about $20 \mathrm{~ms}$ from docking (Domanska et al., 2009).

Further type of a fusion assay involving planar membrane bilayers and liposomes in solution, involves pore-spanning membranes prepared on porous substrates (Höfer and Steinem, 2011; Schwenen et al., 2015). Proteins reconstituted on such a membrane exhibit high mobility (Schwenen et al., 2015), but the delay between docking and fusion is very long (measured in seconds rather than milliseconds; Schwenen et al., 2015).

Another approach used in single vesicle assays is a modification of the initial approach with two liposome populations (Weber et al., 1998), for observation of single fusion events. Two variations of this assay were reported with liposomes either being in 
solution with detection by FCCS (fluorescence cross-correlation spectroscopy; Cypionka et al., 2009) or ALEX (alternating laser excitation; Kim et al., 2012), or with one set of liposomes being immobilised on a surface and second set added in solution (Yoon et al., 2006, see also Figure 1.5). The latter approach (with immobilised vesicles) was extensively developed (Kyoung et al., 2013; Brunger et al., 2015), and currently allows observation of the $\mathrm{Ca}^{2+}$-triggered fusion (Kyoung et al., 2011; Diao et al., 2012; Lai et al., 2014), but still with relatively high, and non-physiological $\mathrm{Ca}^{2+}$ concentrations $(500 \mu \mathrm{M})$. All of the vesicle-based approaches presented in this paragraph, reported rather slow fusion events, also probably due to technical constrains of the used imaging techniques.

Despite the substantial development and wide use of the single vesicle assays, there is still a functional gap between the in vitro approaches and synaptic vesicle fusion observed in cells. First, the $\mathrm{Ca}^{2+}$-triggering is hard to achieve in vitro, and second, the speed of vesicle fusion is usually orders of magnitude slower than in vivo. The reason for the lack of the $\mathrm{Ca}^{2+}$-triggering is that the molecular mechanism is still unknown and therefore cannot be reconstructed in vitro. The fusion speed however, should be determined by the concentration and activation state of SNARE proteins. Despite using various SNARE concentrations and also an artificially activated acceptor complex (see also Section 2.2.3; Pobbati et al., 2006), time from docking to fusion, even in the fastest case, is still at least 10 times slower than in vivo (Domanska et al., 2009). The reason for that may be attachment of the membrane to the surface (in case of planar membrane bilayers as well as immobilised vesicles) that may restrict lateral diffusion of proteins as well as lead to development of stress forces that may affect the energetics of the membrane merger. What is more, in case of fusion of two populations of vesicles, both types of liposomes are highly curved in contrast to the native situation with synaptic vesicles and the plasma membrane. 


\subsection{Aims of this study}

The aim of this study was to investigate the mechanism of SNARE-mediated synaptic vesicle exocytosis. For this purpose a novel single vesicle, GUV-based membrane fusion assay was developed in the course of this work, in order to address specific questions that are remaining in the field of chemical neurotransmission (see questions on page 22). Especially two concerns were emphasised in this study, namely:

1. How fast can SNAREs induce membrane fusion? - This question was addressed by monitoring liposome and chromaffin granule fusion events in a GUV-based assay (Section 3.2).

2. How does directional SNARE zippering translate to formation of membrane fusion intermediates? - This question was addressed by monitoring the docking process of vesicles with SNARE complex assembly mutants $-\Delta 84$ syb and I45A, M46A syb (Hernandez et al., 2012; Yavuz, 2015) — described before to stall liposome fusion at the docked state (Section 3.3).
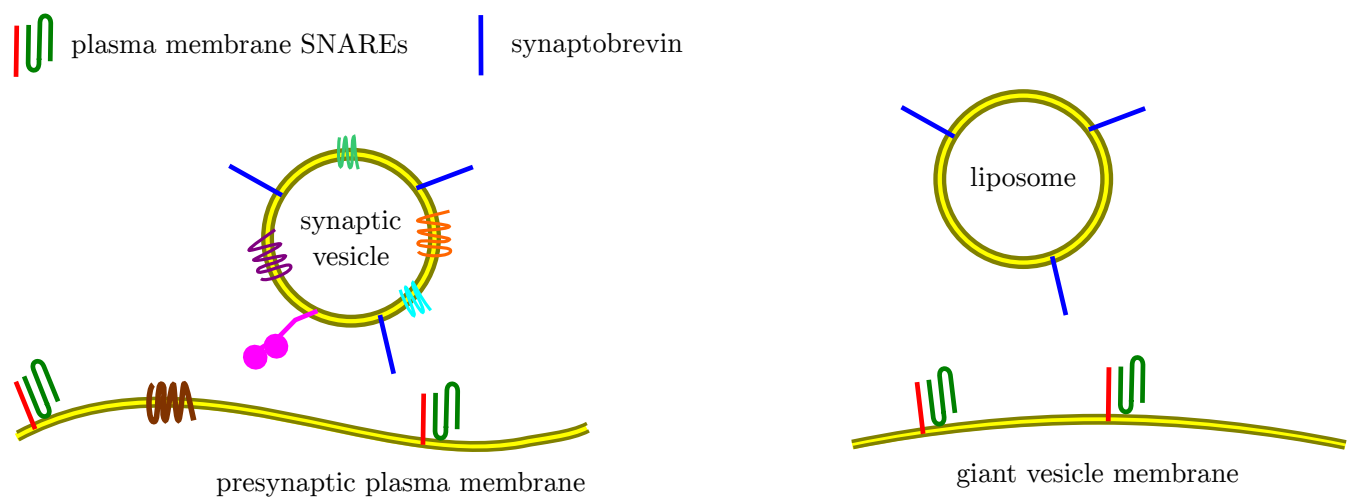

Figure 1.6. Reconstruction of synaptic vesicle exocytosis in an in vitro assay.

In order to access information about various steps of the membrane fusion reaction, single vesicle monitoring is needed. Additionally, for assessment of the influence of specific conditions on the fusion reaction, more simplistic, in vitro approaches are usually a better choice. Up to now, single vesicle, in vitro fusion assays that were described still lack some features observed in vivo (like for example fast fusion speed), and may be prone to artefacts due to surface immobilisation of membranes (except for FCCS and ALEX-based assays that lack possibility of monitoring the reaction at appropriate time resolution, see also Section 1.3.2). Because of that, in this study a new 
GUV-based assay was developed that minimises the surface-related artefacts as well as provides a natural membrane topology for studying synaptic vesicle exocytosis with one of the membranes being relatively flat and the second one highly curved (Figure 1.6). Since GUV system has not been extensively used before for similar types of studies, a large part of this work comprises formation optimisation and characterisation of SNARE containing GUVs (Section 3.1).

The GUV-based assay was designed in a way that it could be easily applied for addressing other biologically important questions that can concern the $\mathrm{Ca}^{2+}$-triggering of synaptic vesicle exocytosis, or the mechanism of other SNARE proteins that take part for example in endosomal fusion. 


\section{Materials and Methods}

\subsection{Materials}

Most of the used chemical reagents were from Sigma-Aldrich or ThermoFisher Scientific. Commercially available reagents were of highest purity grade. Specific reagents are listed in the Appendix Table A.1 (general reagents), Table A.2 (detergents), or in the Table A.3 (lipids).

All detergents listed in Table A.2 except for octyl $\beta$-D-glucopyranoside (OG) are from Anatrace. OG was purchased from Glycon. Additionally, CHAPS was from Anatrace and Triton X-100 was from Merck.

Specific solutions used in this study are listed in the Appendix Table A.6.

Equipment, instruments, and miscellaneous products used throughout this study are listed in the Appendix Table A.4 and Table A.5, and otherwise stated in text. Specification of the microscope setups used is described in Table A.7.

\section{$2.2 \quad$ Protein handling}

\subsubsection{Basic methods}

\section{Bacterial transformation}

Escherichia coli BL21 DE3 cells (electrocompetent from Stratagene/Agilent Technologies) were transformed with plasmids carrying constructs listed in Table 2.1 using a modified protocol from Dower et al. (1988). All used protein constructs were cloned into the NdeI/XhoI restriction sites of the pET28a expression vector (Novagen, 2003). For electroformation, bacteria $(\sim 70 \mu \mathrm{l})$ were thawed on ice and then mixed with 50-100 ng of plasmid DNA in an electroporation cuvette (0.2 cm gap, BioRad). The electroporation was performed with MicroPulser (BioRad) with an electrical pulse $2.5 \mathrm{kV}$. Then, bacteria were mixed with $1 \mathrm{ml}$ of preheated LB medium (Bertani, 1951; without glucose) and incubated at $37^{\circ} \mathrm{C}$ shaking for $\sim 45 \mathrm{~min}$. Afterwards, bacteria were plated on a LB agar plate $(1.8 \% \mathrm{w} / \mathrm{v}$ agar dissolved in the LB medium) with kanamycin, and grown overnight at $37^{\circ} \mathrm{C}$. 
Table 2.1. Protein constructs. All protein sequences were derived from Rattus Norvegicus unless stated otherwise. Constructs were cloned into a pET28a expression vector.

\begin{tabular}{|c|c|c|c|c|}
\hline Abbreviation & Protein name & $\begin{array}{l}\text { Amino } \\
\text { acids } \\
\text { range }\end{array}$ & Mutations & Reference \\
\hline syb $49-96^{a}$ & \multirow{5}{*}{$\begin{array}{c}\text { Vamp-2/ } \\
\text { Synaptobrevin-2 }\end{array}$} & $49-96$ & - & Pobbati et al. \\
\hline syb $1-96$ & & $1-96$ & $\mathrm{~S} 28 \mathrm{C}$ & Margittai et al. \\
\hline WT syb & & $1-116$ & - & Pabst \\
\hline$\Delta 84 \mathrm{syb}$ & & $1-116$ & $\Delta 84$ & Siddiqui et al. \\
\hline $\mathrm{AA} \mathrm{syb}^{a}$ & & $1-116$ & $\mathrm{I} 45 \mathrm{~A}, \mathrm{M} 46 \mathrm{~A}$ & Wiederhold et al. \\
\hline- & \multirow[b]{2}{*}{ SNAP-25 } & $1-206$ & $\begin{array}{l}\text { C84S, C85S, } \\
\text { C90S, C92S }\end{array}$ & Fasshauer et al. \\
\hline- & & $1-206$ & $\begin{array}{c}\text { C84S, C85S, } \\
\text { C90S, C92S, } \\
\text { S130C }\end{array}$ & Margittai et al. \\
\hline syntaxin & Syntaxin-1A & $183-288$ & - & Schuette et al. \\
\hline $\mathrm{NSF}^{a, b}$ & $\begin{array}{c}\text { Vesicle-fusing } \\
\text { ATPase/ } \\
\text { N-ethylmaleimide } \\
\text { Sensitive Fusion } \\
\text { protein }\end{array}$ & $1-744$ & - & Winter et al. \\
\hline$\alpha \mathrm{SNAP}^{a, c}$ & $\begin{array}{c}\text { NAPA/ } \\
\text { SNAP alpha }\end{array}$ & $1-295$ & - & Winter et al. \\
\hline
\end{tabular}

${ }^{a}$ expressed and purified by Ursel Reis or Dr. Halenur Yavuz (Yavuz, 2015)

${ }^{b}$ Chinese hamster sequence (Cricetulus griseus)

${ }^{c}$ bovine sequence (Bos taurus)

\section{Protein expression}

The expression and purification of proteins was done essentially as described in Hernandez (2012) and Yavuz (2015, especially syntaxin purification protocol). For protein expression a colony was picked from a LB agar plate and grown overnight in LB medium at $37^{\circ} \mathrm{C}$. On the next day, this pre-culture was used for a large scale expression in TB medium (initially presented by Tartof and Hobbs, 1987 with further modifications reported in Cold Spring Harbor Protocols, 2006; supplemented with $50 \mu \mathrm{g} / \mathrm{ml}$ kanamycin). Cells were grown shaking at $37^{\circ} \mathrm{C}$ until they reached $\mathrm{OD}_{600} 0.8-1.0$, and 
then expression was induced with $0.5 \mathrm{mM}$ IPTG. Recombinant protein was expressed for $4 \mathrm{~h}$ at $37^{\circ} \mathrm{C}$ and then harvested via centrifugation $\left(20 \mathrm{~min}, 4^{\circ} \mathrm{C}, 4,100 \times g\right)$, resuspended in resuspension buffer (Table A.6, $\sim 100 \mathrm{ml}$ ), and stored at $-20^{\circ} \mathrm{C}$.

\section{Protein purification}

For protein extraction and purification bacteria were thawed and subsequently the extraction buffer (Table A.6, $100 \mathrm{ml}$ ) supplemented with lysozyme $(4 \mathrm{mg} / \mathrm{l}$ of initial culture), $\mathrm{MgCl}_{2}$ (1 mM), DNaseI (tip of a spatula), protease inhibitor cocktail tablet (cOmplete, EDTA-free, Roche) was added. Afterwards, this mixture was incubated for $15 \mathrm{~min}$ at room temperature. Cells were then lysed with a sonicator (Branson Sonifier 450, microtip limit, $50 \%$ duty cycle, $4 \times 40$ strokes), supplemented with $6 \mathrm{M}$ urea, and incubated for around 15 min. Protein was later harvested by centrifugation $\left(60 \mathrm{~min}, 4^{\circ} \mathrm{C}, 25,000 \times g\right)$ and then incubated for at least $2 \mathrm{~h}$ with Ni-NTA agarose beads at $4^{\circ} \mathrm{C}$ (Qiagen, $12 \mathrm{ml}$ beads per 61 culture). Beads with bound His-tagged protein were collected on Econo-Column (BioRad), washed with the wash buffer and protein was eluted with the elution buffer containing concentrated imidazole (both solutions in Table A.6). After overnight dialysis (dialysis buffer, Table A.6, supplemented with thrombin for the His-tag cleavage $5 \mathrm{mg} / \mathrm{ml}$ in $50 \%$ glycerol, $1 \mathrm{U} / \mathrm{\mu l}, 100 \mathrm{ml}$ for $5 \mathrm{ml}$ of protein solution; MWCO $8 \mathrm{kDa}$ ), protein was further purified by ion exchange chromatography (with the ÄKTA system) using a $\mathrm{NaCl}$ gradient (from $50 \mathrm{mM}$ to $500 \mathrm{mM}$ ) for elution (buffer with HEPES $20 \mathrm{mM}$, pH 7.4, DTT $1 \mathrm{mM}$, EDTA $1 \mathrm{mM}$, and supplemented with OG for syb 1-116 and CHAPS for syntaxin). Depending on the protein isoelectric point (determined in ExPASy database, Gasteiger et al., 2005), anion exchanger (for syntaxin and SNAP-25) or cation exchanger (for synaptobrevin) was used. Protein purity and His-tag cleavage efficiency was evaluated by Tricine-SDS-PAGE followed by Coomassie blue staining (see section Tricine-SDS-PAGE and Coomassie blue staining). Fraction concentration was calculated based on the absorbance at $280 \mathrm{~nm}$ (NanoDrop 1000 spectrophotometer, Thermo Scientific), and extinction coefficients and molecular weight determined by ProtParam tool available in the ExPASy database (Gasteiger et al., 2005) with the Beer-Lambert law (Equation 1). Aliquoted protein was then snap frozen in liquid nitrogen and stored at $-80^{\circ} \mathrm{C}$ until use. 


\section{Tricine-SDS-PAGE and Coomassie blue staining}

Proteins were analysed by electrophoresis with Tricine-SDS-PAGE according to the protocol by Schägger and von Jagow (1987) with following modifications. Stacking and separating gel contained $4 \%$ and $10 \%$ acrylamide, respectively, and the electrophoretic run was performed at $60 \mathrm{~V}$ for $15 \mathrm{~min}$ followed by $120 \mathrm{~V}$ for $\sim 45 \mathrm{~min}$. For visualisation the polyacrylamide gel was stained with a Coomassie blue by briefly boiling the gel in the Coomassie solution in the microwave and subsequent $\sim 5$ min incubation in the room temperature. Next, the gel was destained in 2 steps by gently shaking for $5 \mathrm{~min}$ in the destaining solution 1 and for several hours in the destaining solution 2 (Table A.6).

\subsubsection{Protein labelling}

Fluorescent dyes (Table A.1) were coupled to single cysteine mutants of syb 1-96 and SNAP-25 according to the manufacturers instructions (Molecular Probes, 2006). For the Oregon Green 488 (OG488) iodoacetamide, methanol was used as a solvent, and for the Texas Red (TR) maleimide, DMSO was utilised. Dyes were added in 6-10× molar excess to the protein solution (with DTT dialysed out) and incubated for $2 \mathrm{~h}$ at room temperature. The unreacted dye species were then removed by size exclusion on PD-10 columns. Dye concentration $(c)$ was calculated with the Beer-Lambert law (Equation 1), by measuring dye absorbance $(A)$ at the absorption maximum wavelength on NanoDrop 1000, and with light path length $(l)$ and dye extinction coefficient $(\varepsilon)$.

$$
A=\varepsilon c l \Rightarrow c=\frac{A}{\varepsilon l}
$$

Protein concentration was determined with a Pierce $660 \mathrm{~nm}$ Protein Determination Kit according to manufacturers instructions (Pierce Biotechnology, 2013), with absorbance readout on a microplate reader (Genios Pro, TECAN). Degree of labelling (DOL, Equation 2$)$ was obtained by comparison of dye $\left(c_{\text {dye }}\right)$ and protein $\left(c_{\text {protein }}\right)$ concentration in the final fractions.

$$
\mathrm{DOL}=\frac{c_{\text {protein }}}{c_{\text {dye }}}
$$




\subsubsection{SNARE acceptor complex formation}

SNARE acceptor complex (the so called $\Delta \mathrm{N}$ complex) consisting of SNAP-25, syntaxin lacking its N-terminal domain, and syb 49-96 was assembled from purified monomers as described in Pobbati et al. (2006) with the use of OG as in Hernandez et al. (2012). Briefly, monomers were mixed in the molar ratio 1:1:1.5, respectively, and the OG concentration was adjusted to $1 \%(\mathrm{w} / \mathrm{v})$. After overnight incubation, complex was purified by ion exchange chromatography with anion exchanger in the presence of $1 \%(\mathrm{w} / \mathrm{v})$ OG with a 2-step linear $\mathrm{NaCl}$ gradient (complex eluted at $\sim 300-400 \mathrm{mM}$ salt). Purity of the complex was assessed by analysis of unboiled protein sample with Tricine-SDSPAGE and Coomassie blue staining, and activity was checked by determination of syb 1-96 (labelled with OG488) binding efficiency with fluorescence anisotropy measurement (as described in Section 2.4). The purified complex was then snap frozen and stored at $-80^{\circ} \mathrm{C}$ until use. Labelled complex was essentially formed in the same way, with the exception of SNAP-25 being replaced with a S130C mutant coupled to Texas Red.

\subsection{Lipid vesicle preparation}

Lipid mixtures used for preparation of liposomes were prepared from PC, PE, PS and cholesterol mixed in a ratio of 5:2:2:1, respectively. Labelled lipids, biotinylated lipids, or lipophilic tracers were incorporated by replacing a portion of PC (or PE in case of PE-labelled species) with usually $1 \mathrm{~mol} \%$ of respective labelled molecule $(1.5 \mathrm{~mol} \%$ were used for NBD/Rhodamine FRET experiments). Synaptobrevin was reconstituted in a protein to lipid ratio $1: 500$ and the $\Delta \mathrm{N}$ complex 1:1000, unless stated otherwise.

\subsubsection{Small unilamellar vesicles}

Small unilamellar vesicles (SUVs) were prepared essentially like described in Pobbati et al. (2006) with some modifications, and according to the co-micellisation method (Allen et al., 1980). Lipid mix was dried under the nitrogen stream and then resuspended in a liposome buffer supplemented with $5 \%(\mathrm{w} / \mathrm{v})$ sodium cholate at a total lipid concentration of $13.5 \mathrm{mM}(50-100 \mu \mathrm{l})$. After addition of the SNARE proteins, the mixture was subjected to a size-exclusion chromatography (Sephadex G-50 superfine resin, equilibrated with liposome buffer). Eluate drops were illuminated with 
a laser pointer. Liposome-rich fraction was identified as the most light-scattering fraction $(\sim 500 \mu \mathrm{l})$. SUVs were collected and stored for up to 5 days in the fridge until use.

\subsubsection{Large unilamellar vesicles}

Large unilamellar vesicles (LUVs) were prepared based on the protocol by Düzgüneş (2003) as described in Hernandez et al. (2012) with small modifications. Lipid mix was prepared in a pear-shaped flask $(5 \mathrm{ml})$ previously purged with argon. Solvent was removed with a rotary evaporator (BÜCHI Rotavapor R-124) by gradually lowering the pressure to $50 \mathrm{mbar}(\sim 45 \mathrm{~min})$. Afterwards, the lipid film was dissolved in diethyl ether and then, the liposome buffer was added (diethyl ether to liposome buffer ratio $3: 1$ ) in volume that would make $8 \mathrm{mM}$ final lipid concentration after organic solvent evaporation. The resulting two-phase mixture was dispersed by sonication (Branson Sonifier, fine tip, $50 \%$ duty cycle at minimum intensity) $3 \times 45 \mathrm{~s}$ with at least $45 \mathrm{~s}$-long breaks with cooling on ice. Subsequently, diethyl ether was removed by reverse-phase evaporation by gradually lowering the pressure to $25 \mathrm{mbar}(\sim 90 \mathrm{~min})$. The resulting solution that contained multilamellar vesicles (volume again adjusted to $8 \mathrm{mM}$ final lipid concentration) was then extruded (Mini-Extruder, Avanti Polar Lipids) by passing the vesicle solution multiple times $(\sim 25 \times)$ through polycarbonate membranes with pore sizes of 400 and then $100 \mathrm{~nm}$.

Reconstitution of SNARE proteins was obtained with a slightly modified method from Hernandez et al. (2012) based on a procedure from Rigaud and Lévy (2003), essentially following the protocol described in Yavuz (2015). The SNARE protein insertion is highly dependent on the ratio of lipids and detergents (the $R$-value) as explained in Hernandez et al. (2012), with the $R$-values defined by Equation 3 (Rigaud and Lévy, 2003):

$$
R=\frac{\left[\mathrm{D}_{\text {total }}\right]-\left[\mathrm{D}_{\mathrm{CMC}}\right]}{[\text { lipid }]}
$$

where $\left[\mathrm{D}_{\text {total }}\right]$ and $\left[\mathrm{D}_{\mathrm{CMC}}\right]$ denote total and CMC concentrations of the detergent, respectively; and [lipid] is the lipid concentration. The liposomes, protein, OG and liposome buffer were mixed to obtain lipid concentration of $4 \mathrm{mM}$ and $R$-value of 1.5 for syb and 2.0 for the $\Delta \mathrm{N}$ complex reconstitution, assuming $5.5 \mathrm{mM}$ lipid concentration in the liposome solution (due to lipid loss during extrusion) and CMC of OG $17 \mathrm{mM}$ 
(Rigaud and Lévy, 2003). The volume of prepared liposomes varied between 100 and $500 \mathrm{\mu l}$. This mixture was then dialysed in 2 steps (overnight and then in the 2 nd buffer $\sim 3 \mathrm{~h}, 2 \mathrm{l}$ buffer volume) against the liposome buffer containing $2 \mathrm{~g} / \mathrm{l}$ of Bio-Beads during the first dialysis to remove the detergent. Depending on the volume of prepared liposomes either dialysis cassettes $(0.2-0.5 \mathrm{ml})$ or MINI dialysis devices $(\leq 100 \mu \mathrm{l})$ were used, both with 2000 MWCO. LUVs containing reconstituted proteins were stored for up to 5 days in the fridge until use.

\subsubsection{Giant unilamellar vesicles}

Formation of GUVs containing reconstituted proteins is known to be very demanding, although it has been performed in the past (see e.g. Ajouz et al., 2000; Girard et al., 2004; Doeven et al., 2005; Aimon et al., 2011). The most traditional method was to dehydrate native membranes or proteoliposomes with a protein of interest along with exogenous lipids and rehydrate them (Criado and Keller, 1987; Ajouz et al., 2000). However, such a procedure, probably due to not-well-controlled rehydration process, was known to be highly inefficient and yield a very heterogeneous population of giant vesicles (Girard et al., 2004). To solve this problem, the procedure of GUV formation was then further improved by implementation of electroformation technique (Angelova et al., 1992) being successfully used for formation of GUVs containing bacteriorhodophsin (Manneville et al., 1999). Still, this method utilised organic solvents that could potentially denature many proteins other than bacteriorhodophsin and therefore was not suitable for other preparations. Finally, a procedure of GUV formation directly from dried proteoliposomes was introduced (Girard et al., 2004; Doeven et al., 2005). In this method, preformed proteoliposomes were dried on the surface of conductive indium tin oxide (ITO) glass slides (or platinum electrodes) and afterwards electroformation chamber (see Figure 2.2) is filled with a rehydration buffer. Such chamber is then connected to a function generator that supplies an $\mathrm{AC}$ electric field according to an appropriate protocol (voltage, time and frequency, Angelova et al., 1992). The main disadvantages of this method that may influence protein activity include (i) drying of the protein that is incorporated in the proteoliposomes and that (ii) rehydration buffer for most successful electroformation protocols contains no salt. Although, there have been continuous efforts to overcome these disadvantages (Doeven et al., 2005; Pott et al., 2008; Aimon et al., 2011; Dezi et al., 2013), it turns out that protocols for successful formation of giant proteoliposomes vary depending on (i) lipid composition, (ii) reconstituted protein, (iii) buffer composition, (iv) resistance 
and quality of conductive slides (or platinum electrodes) used, and finally (v) chamber design (i.e. volume, spacer thickness, electrode surface). In practice it means that the proteo-GUV formation protocol has to be re-optimised each time for new set of parameters, described earlier.

SUV preparation

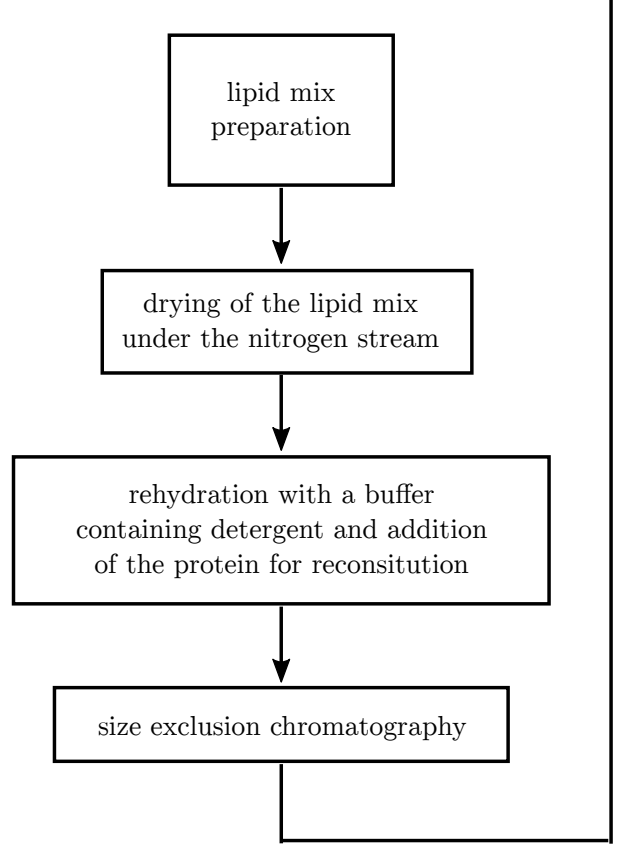

GUV preparation

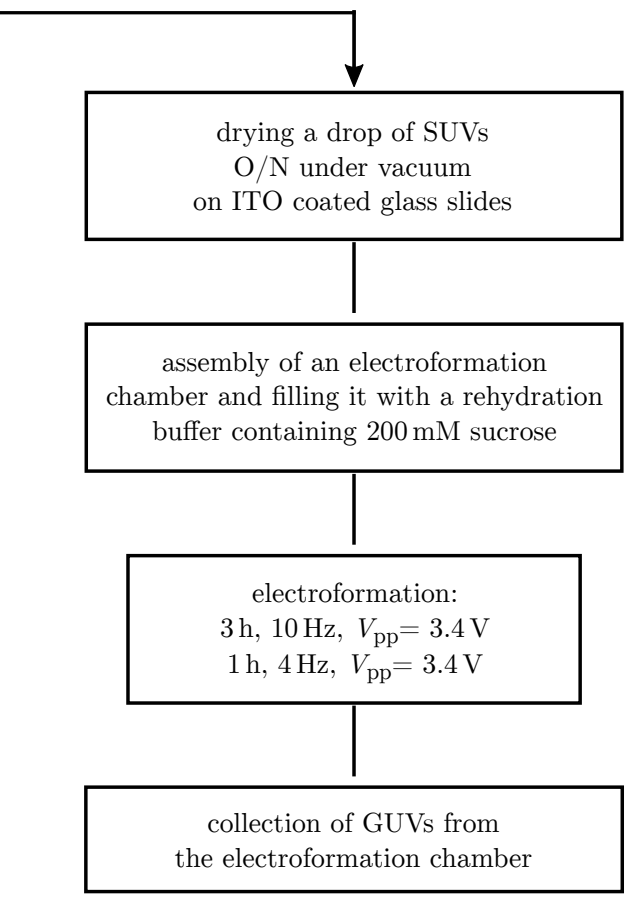

Figure 2.1. GUV preparation workflow. Flowchart presenting a workflow for the $\triangle \mathrm{N}-\mathrm{GUV}$ preparation.

The formation procedure of giant unilamellar vesicles (GUVs) containing reconstituted $\Delta \mathrm{N}$ complex had to be optimised in the course of this study, and is described in Section 3.1.1 and the workflow is presented in Figure 2.1. In the final form, protocol resembled one reported by Bacia et al. (2004) and was done with an electroformation technique (Angelova et al., 1992). For all experiments except for iSCAT microscopy measurements, GUVs contained 1 mol\% of biotinylated-PE (Section 2.5.3). GUVs were formed by drying a drop of $\triangle \mathrm{N}-\mathrm{SUVs}$ on indium tin oxide (ITO) coated glasses (Table A.4) overnight in an exsiccator. Afterwards, an electroformation chamber (Figure 2.2) was assembled and completely filled with $200 \mathrm{mM}$ sucrose solution $(\sim 600 \mu \mathrm{l})$ with the use of a thin needle and a syringe. Electroformation was performed with a function generator (PCGU1000, Velleman) operated by software PcLab2000SE (Velleman) on a Windows XP computer. Sinusoidal alternating electric field was applied for 
$3 \mathrm{~h}\left(V_{\mathrm{pp}}=3.4 \mathrm{~V}, 10 \mathrm{~Hz}\right)$ followed by a detachment phase for around $1 \mathrm{~h}\left(V_{\mathrm{pp}}=3.4 \mathrm{~V}\right.$, $4 \mathrm{~Hz}$ ). Finally, the chamber was disassembled, GUVs were collected for further experiments, and stored for up to 5 days in the fridge.



Figure 2.2. Electroformation chamber design. Schematic representation of an electroformation chamber used for GUV formation. SUVs are dried on the glass surface in regions marked in blue before the chamber assembly. The electroformation chamber is assembled from two glasses (coated on the one side with ITO), directed with conductive sides towards each other and with a $3 \mathrm{~mm}$-thick ring-shape spacer (light grey). The rehydration buffer (200 mM sucrose) is filled in the chamber with a syringe by puncturing the silicone spacer with a thin needle. The function generator is connected to the glasses with crocodile clips, through a stripe of a copper tape (dark grey stripes) attached to glass with conductive glue (Table A.5). Based on the original chamber design presented in Angelova et al. (1992).

For iSCAT microscopy experiments, GUVs were prepared with essentially the same protocol with the exception of platinum electrodes (Angelova and Dimitrov, 1986) that were used instead of ITO slides, because this method yielded a bit larger GUVs. For these experiments $\Delta \mathrm{N}-\mathrm{SUVs}$ were prepared in the Max Planck Institute for Biophysical Chemistry in Göttingen (Germany) and then they were transported to the Max Planck Institute for the Science of Light in Erlangen (Germany) and subjected to GUV preparation procedure there. All experiments performed in Erlangen were done together with Susann Spindler (from the institute in Erlangen, group of Prof. Vahid Sandoghdar). 


\subsubsection{Chromaffin granule purification and labelling}

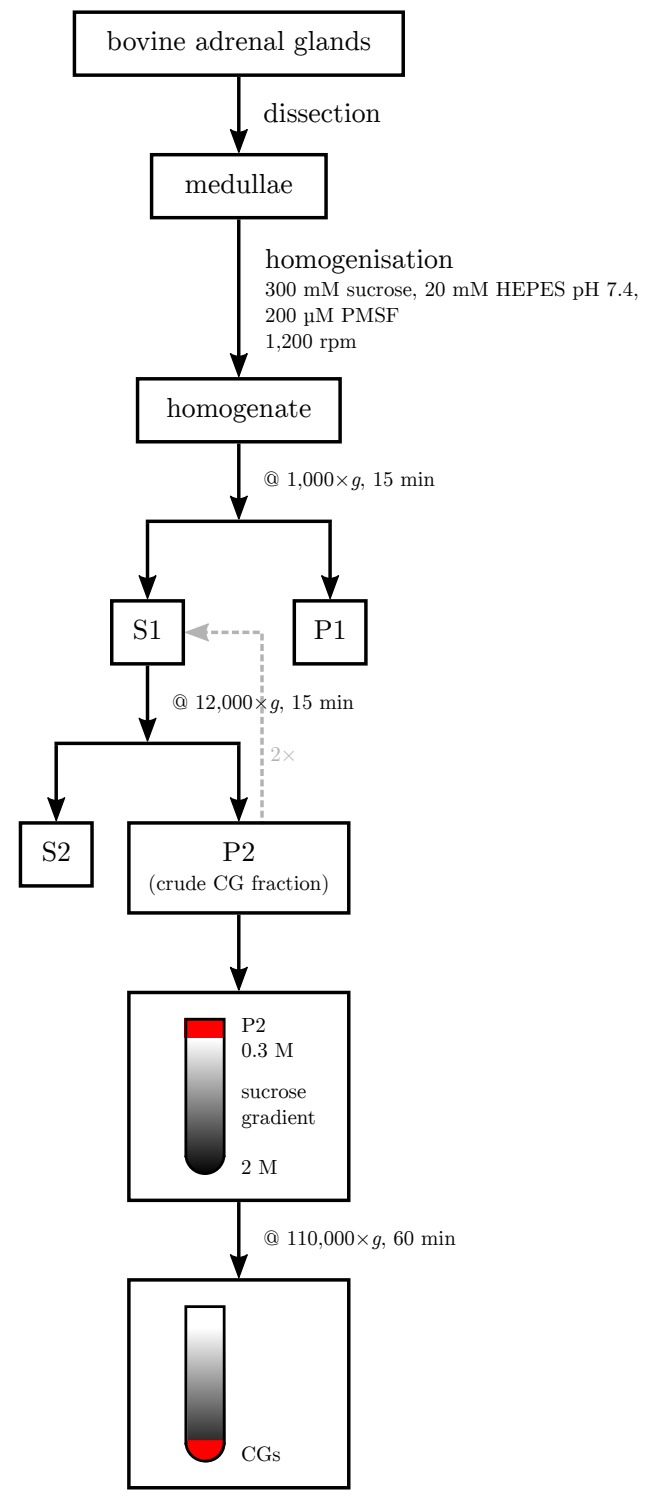

Figure 2.3. Chromaffin granule purification procedure. Diagram schematically presenting protocol used for the chromaffin granule purification according to Park et al. (2012b). Centrifugation steps are indicated with an "@" symbol. Description in text.

Chromaffin granules (CGs) were purified from bovine adrenal gland medullae with a density gradient centrifugation method (Figure 2.3) as described in Park et al. (2012b) and based on protocol by Smith and Winkler (1967). Fresh bovine adrenal glands were obtained on ice from a local slaughterhouse and all subsequent steps were performed at $4{ }^{\circ} \mathrm{C}$. After dissection, medullae were minced with scissors and subsequently homogenised using a cooled glass-Teflon homogeniser at 1,200 rpm in 300-mM sucrose 


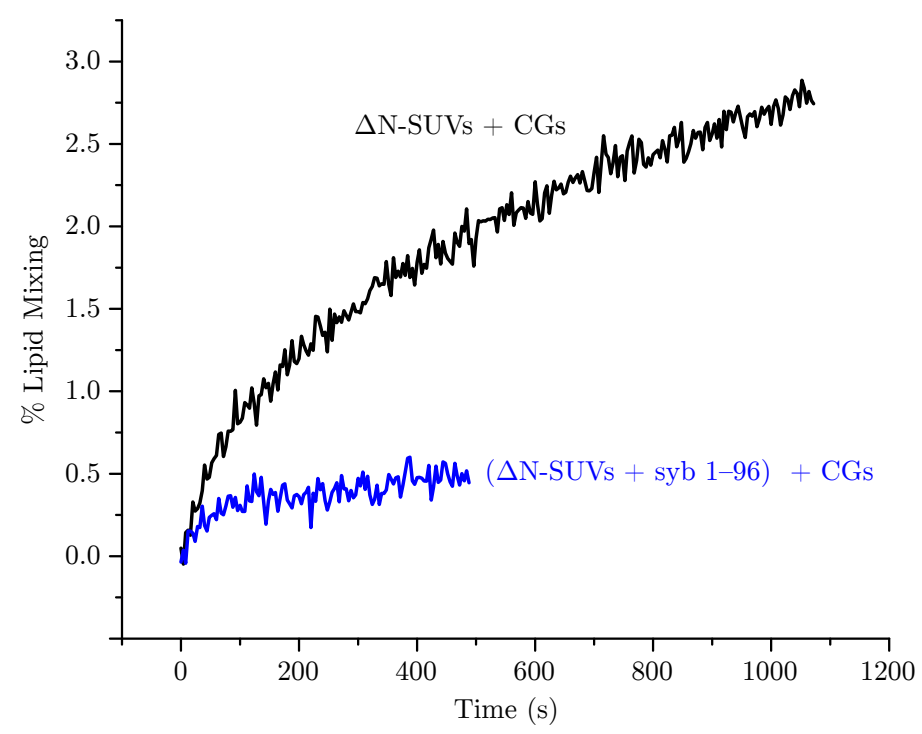

Figure 2.4. Activity control for the purified chromaffin granules. Purified chromaffin granules fuse with $\Delta \mathrm{N}$ liposomes. Test performed as described in Park et al. (2012b) with a lipid mixing method (Section 2.5.8). $10 \mu \mathrm{l}$ of CGs were added to $10 \mu \mathrm{l}$ NBD-Rho labelled $\Delta N$-LUVs and NBD dequenching was monitored (black trace). Control was done with $\Delta \mathrm{N}$ liposomes preincubated with molar excess of soluble syb 1-96 (blue trace). Measurement was performed in $37^{\circ} \mathrm{C}$ in Fluoromax 2 spectrophotometer (Jobin Yvon) by monitoring fluorescence with excitation at $460 \mathrm{~nm}$ and emission at $538 \mathrm{~nm}$. The $\%$ of lipid mixing was calculated by normalising to the maximum NBD signal created after addition of $0.1 \%$ Triton X-100 that dispersed all lipids present in the sample.

buffer (300 mM sucrose, 20 mM HEPES pH 7.4 adjusted with KOH, supplemented with $200 \mathrm{\mu M}$ of protease inhibitor PMSF). The sample was then centrifuged at $1,000 \times g$ for 15 min and pellet (P1) was discarded. The supernatant (S1) was further centrifuged at $12,000 \times g$ for $15 \mathrm{~min}$, resuspended and washed for 2 more rounds. Pellet (P2) was then resuspended in 300-mM sucrose buffer and loaded on top of a continuous sucrose gradient (from $300 \mathrm{mM}$ to $2 \mathrm{M}$ sucrose) and further centrifuged at $110,000 \times \mathrm{g}$ for $60 \mathrm{~min}$. CGs were then resuspended from the pellet in liposome buffer, snap frozen in liquid nitrogen and stored at $-80^{\circ} \mathrm{C}$ until use. Additionally, after purification, fusogenic activity of CGs was checked by monitoring fusion-mediated dequenching of NBD coming from NBD-Rho labelled $\Delta \mathrm{N}$ liposomes (Figure 2.4, lipid mixing assay principle described in Section 2.5.8).

Chromaffin granule preparation and activity control was performed together with Dr. Yongsoo Park (Göttingen, now Izmir, Turkey) according to his optimised protocol (Figure 2.3). 
Prior to use, CGs were thawn on ice, added to a dried lipid film consisting of TR-PE, and subsequently incubated $30 \mathrm{~min}$ in $37^{\circ} \mathrm{C}$ shaking (method modified from Kiessling et al., 2013). This procedure resulted in fluorescently labelled granules that could be visualised under microscope as can be seen in Figure 3.16.

\subsection{Bulk fluorescence anisotropy assay used for monitoring of the ternary SNARE complex formation}

Fluorescence anisotropy of a fluorophore reports the polarisation of the emitted light when a fluorophore was excited with a polarised light. The anisotropy value depends on the preferred orientation of the fluorophore and can be therefore used to asses the conformational motion of the labelled residue in a protein of interest reporting on events like complex formation or complex disassembly (Lakowicz, 2006). Anisotropy is calculated from the following equation:

$$
r=\frac{I_{\mathrm{VV}}-G I_{\mathrm{VH}}}{I_{\mathrm{VV}}+2 G I_{\mathrm{VH}}}
$$

where $I_{\mathrm{VV}}$ and $I_{\mathrm{VH}}$ denote fluorescence intensities of the vertically and horizontally polarised emissions of the fluorophore with vertically polarised excitation light, and $G$ is a correction factor depending on the instrument that is calculated from:

$$
G=\frac{I_{\mathrm{HV}}}{I_{\mathrm{HH}}}
$$

with $I_{\mathrm{HV}}$ and $I_{\mathrm{HH}}$ denoting fluorescence intensities of the vertically and horizontally polarised emissions of the fluorophore with horizontally polarised excitation light.

Fluorescence anisotropy of OG488 attached to syb 1-96 S28C (Figure 3.2, 3.3 and 3.7) was measured on Fluorolog 3 spectrophotometer equipped with magnetic stirring, a temperature controller set to $37^{\circ} \mathrm{C}$, and with built-in polarisers (Table A.4). The excitation and emission wavelength was set to 488 and $520 \mathrm{~nm}$, respectively; the integration time set to $2 \mathrm{~s}$, and $G$-factor (Equation 5) was measured separately for each set of experiments. Measurement was performed in the liposome or disassembly buffer (600 $\mathrm{\mu l}$, both buffers listed in Table A.6) with 100-200 nM syb 1-96 OG488 by adding $100 \mu \mathrm{l}$ of $\Delta \mathrm{N}-\mathrm{GUV}$ solution (final lipid concentration $5-15 \mu \mathrm{M}$ ). For the disassembly measurements (Figure 3.7), reaction mixture contained additionally aSNAP $(1 \mu \mathrm{M})$ and NSF (90 nM). 


\subsection{Microscopy: imaging and analysis}

\subsubsection{Fluorescence imaging}

The microscopy setups used for the fluorescence imaging are described in Table A.4 and Table A.7. The custom-build imaging chamber consists of the coverslip holder, that is reusable, and a functionalised coverslip (see Section 2.5.3).

\subsubsection{The iSCAT microscopy}

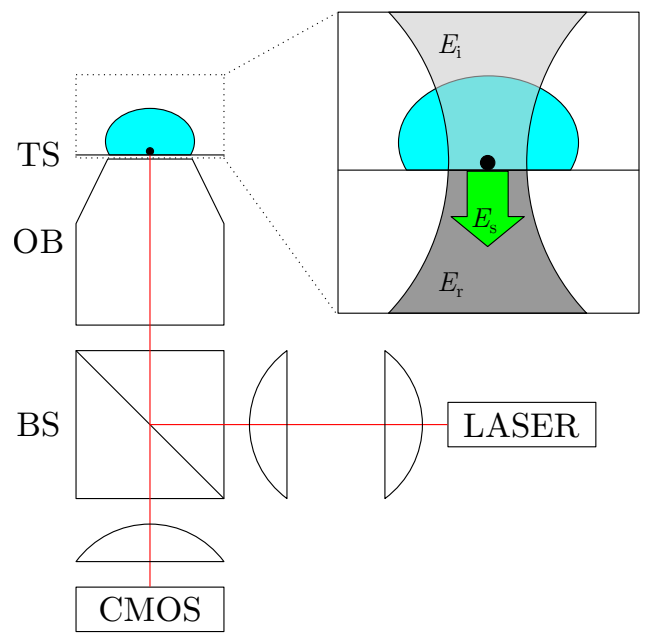

Figure 2.5. The iSCAT microscopy principle. Diagram schematically presenting the principle of interferometric scattering microscopy. The basic iSCAT setup consists of: a translation stage (TS), an objective (OB), a beamsplitter (BS), a CMOS camera (CMOS) and a laser. Inset presents the principle of signal generation: the incident light $\left(E_{\mathrm{i}}\right)$ is focused by the objective on the sample, while the signal is generated by the light reflected $\left(E_{\mathrm{r}}\right)$ at the coverslip interface and by the light backscattered by particles that are being illuminated $\left(E_{\mathrm{s}}\right)$. Based on Ortega-Arroyo and Kukura (2012).

The interferometric scattering (iSCAT) microscopy (Lindfors et al., 2004; Kukura et al., 2009; Hsieh et al., 2014; Ortega-Arroyo and Kukura, 2012) was developed in the group of Prof. Vahid Sandoghdar (Max Planck Institute for the Science of Light in Erlangen, Germany) and allows very fast, label-free, 3D imaging, with very accurate particle localisation (Ortega-Arroyo and Kukura, 2012). The basic principle of this type of microscopy is that instead of relaying on the fluorescence emission of a fluorophore it collects the light scattered by the imaging object along with the reference beam reflected by the coverslip surface. Interference of these two signals creates an image (Figure 2.5). Since the collected signal is not dependent on the number of photons 
emitted by the fluorophore, the imaging rate is not limited by that. Additionally, also $z$-position of the imaged object can be determined based on the differences in the optical path length and resulting change in the overall phase (visible as amplitude modulation, Krishnan et al., 2010; see also Figure 3.21 B).

All experiments with iSCAT were performed together with Susann Spindler (research group of Prof. Vahid Sandoghdar) on her home-built iSCAT microscopy setup at the Max Planck Institute for the Science of Light in Erlangen (Germany). GUVs for these experiments were prepared with the platinum electrode method (see Section 2.3.3) and instead of being immobilised at the coverslip surface were held in place by gentle aspiration with a micropipette (Figure $3.21 \mathrm{~A}$ ). The imaging rate was set to $1 \mathrm{kHz}$.

\subsubsection{Immobilisation of GUVs}

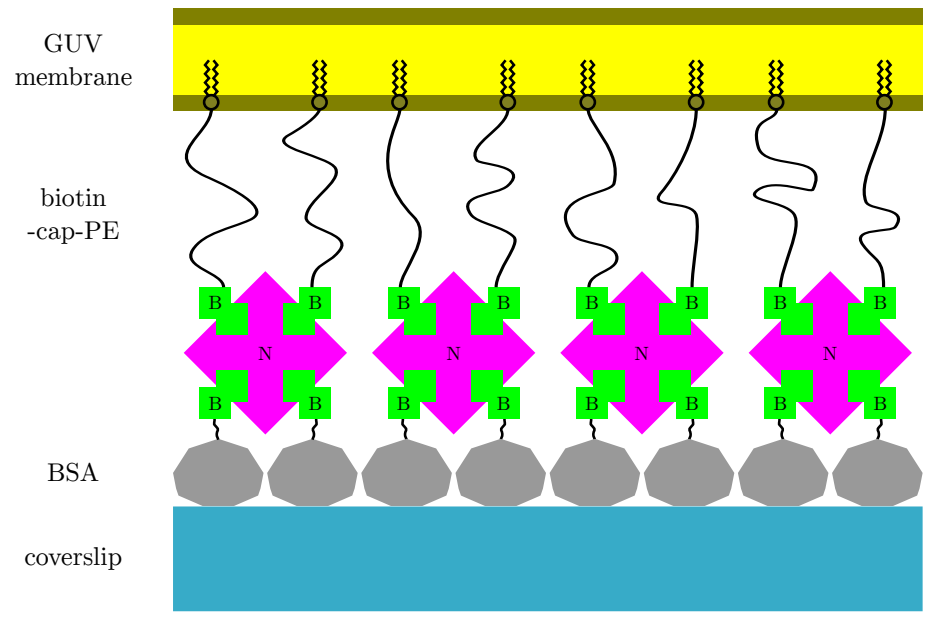

Figure 2.6. GUV immobilisation principle. Schematic illustration presenting the GUV immobilisation method. Clean coverslips are coated with biotinylated BSA (biotin marked with B) and then with NeutrAvidin (N). GUVs are immobilised by interaction of biotinylated lipids (biotin-cap-PE) incorporated into the GUV membrane, with NeutrAvidin attached to the coverslip. Based on the illustration from Huber and Sakmar (2011).

New glass coverslips (for details see Table A.4) were cleaned by bath sonication in the $2 \%(\mathrm{v} / \mathrm{v})$ of Hellmanex II solution (Table A.1) for $20 \mathrm{~min}$, followed by thorough washing in Milli-Q water (at least $3 \times$ ), and additional 10 min of bath sonication also in water. Glass coverslips were coated with biotinylated bovine serum albumin $(2 \mathrm{mg} / \mathrm{ml})$ for $1 \mathrm{~h}$, and subsequently with NeutrAvidin solution $(0.7 \mathrm{mg} / \mathrm{ml}$ in liposome buffer, $1 \mathrm{~h})$. 100-200 $\mathrm{\mu l}$ GUV solution, diluted with $300 \mu \mathrm{l}$ liposome buffer containing $1 \mathrm{mM} \mathrm{MgCl}_{2}$ 
for better adhesion (this phenomenon was described in detail for GUVs in a recent publication by Gleisner et al., 2016), were introduced into an imaging chamber equipped with a coated coverslip on the bottom. Around $30 \mathrm{~min}$ incubation was sufficient for GUVs to settle and become immobilised at the coverslip surface (Figure 2.6). The concentration of biotinylated lipids in the GUVs had to be optimised (Figure 2.7), and $1 \mathrm{~mol} \%$ was found to be the most optimal concentration

biot-PE $(\operatorname{mol} \%)$

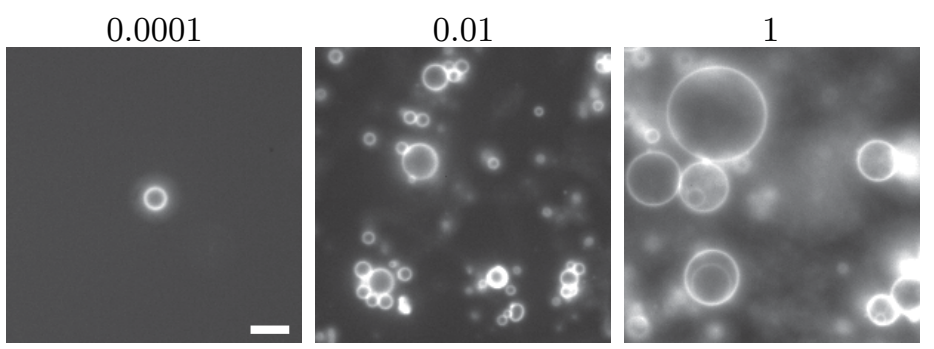

Figure 2.7. Optimisation of biotinylated lipid concentration needed for GUV immobilisation. Representative images showing optimisation of biot-PE concentration. Protein free GUVs were prepared directly from a lipid mix with a short electroformation protocol (Table 3.1). Membrane visualisation was obtained by supplementing liposome buffer with TMA-DPH (Table A.3). Images show the extent of GUV immobilisation on NeutrAvidin functionalised coverslips after 20 min incubation followed by $3 \times$ washing with liposome buffer. Scale bar $10 \mu \mathrm{m}$. Note that higher biot-PE concentrations (e.g. $5 \mathrm{~mol} \%$ ) led to GUV bursting due to probably too much tension. Best immobilisation was obtained with $1 \mathrm{~mol} \%$.

\subsubsection{Design of the GUV-based fusion assay}

The GUV-based fusion assay for investigation of neuronal SNARES (Witkowska and Jahn, 2016) involves GUVs containing reconstituted plasma membrane SNARE proteins (in this work the $\Delta \mathrm{N}$ complex) that are immobilised in the imaging chamber, and liposomes or purified secretory vesicles (in this work SUVs, LUVs and chromaffin granules) containing vesicular SNARE - synaptobrevin 2 (Figure 2.8). First, the unilamellar GUV was selected on a coverslip (as described in Witkowska and Jahn, 2016; vesicles that were not unilamellar, i.e. did not have uniform membrane fluorescence, showed a higher fluorescence intensity than other GUVs of similar size, or contained luminal inclusions, were excluded from image acquisition and data analysis) and microscope focus was positioned at the equatorial plane of the selected GUV. Then, usually just before image acquisition liposomes or chromaffin granules were added to the imaging chamber by pipetting directly on top of the selected GUV. For the flu- 
orescence imaging, membrane lipids, vesicle content, or protein can be labelled with a fluorophore.

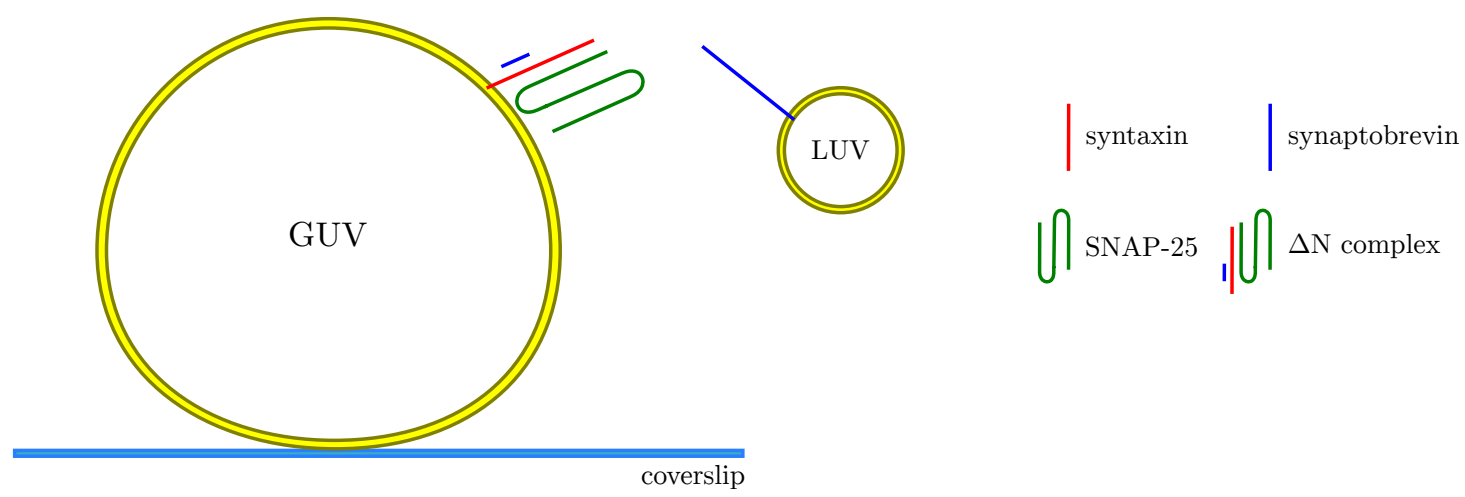

Figure 2.8. A GUV-based fusion assay design. Schematic illustration of a GUVbased fusion assay developed in this study. Description in text.

\subsubsection{Basic analysis of microscopy data}

All images were primarily analysed and normalised with Fiji (Schindelin et al., 2012). The analysis involved adjustment of the brightness and contrast of the lookup table. Background fluorescence correction was performed where indicated, by subtraction of an averaged fluorescence intensity value taken form a region of interest being outside of the visible GUVs. Some images (Figure 3.14, 3.15, and 3.16) were processed with a bicubic extrapolation algorithm (available in Fiji) for better visualisation. However, for generation of the intensity profiles (Figure 3.14 and 3.16), raw images were taken.

For quantification of the GUV membrane fluorescence intensity (see also Section 2.5.6), as well as for the generation of GUV membrane fluorescence intensity profiles (Figure 3.14 and 3.16) a macro was developed (Script 1, inspired by a description from Kurps et al., 2014) that involved linearisation of the GUV membrane. With this method, GUV was identified on an image and was subsequently transformed to polar coordinates with the Polar Transformer plugin installed in Fiji. Such linearised membrane was then either analysed for average membrane fluorescence intensity (Script 1) or an intensity profile was created (e.g. Figure 3.14). For the fluorescence intensity profiles (Figure 3.14 and 3.16), images taken before transformation were normalised by subtracting an averaged stack image from every time frame. The line profiles were extracted from around $1 \mu \mathrm{m}$-thick region of interest containing the linearised GUV membrane. 
Further analysis of numerical data (e.g. frequency counts, curve fitting) was usually performed with Origin software (OriginLab) unless stated otherwise. Average values are presented \pm SEM.

\subsubsection{GUV reconstitution efficiency determination}

The concentration of $\Delta \mathrm{N}$ complexes in the GUV membrane $\left(C_{\text {prot }}\right)$ was determined as described in Aimon et al. (2011) with some modifications (adapted from Witkowska and Jahn, 2016):

$$
C_{\text {prot }}=\frac{I_{\mathrm{mp}}}{N_{\mathrm{f}} \times M_{\mathrm{ref}} \times F}
$$

where $I_{\mathrm{mp}}$ is the membrane fluorescence intensity of the protein, $N_{\mathrm{f}}$ is the number of fluorescent dyes attached to one protein molecule, $M_{\text {ref }}$ is a calibration factor, and $F$ is the calibration scaling factor.

In this method, membrane fluorescence intensity of the TR labelled complex was compared with that of a reference fluorophore (TR-PE), expressed with a calibration factor $M_{\text {ref: }}$ :

$$
M_{\mathrm{ref}}=\frac{\sum_{i=1}^{n} \frac{I_{\mathrm{ml}}}{C_{\mathrm{ml}}}(i)}{n}
$$

that is calculated from a calibration curve (Figure 2.9) generated from membrane fluorescence intensities $\left(I_{\mathrm{ml}}\right)$ of $n$-number of varying concentrations of TR-PE $\left(C_{\mathrm{ml}}\right)$.

Additionally, intrinsic properties of a dye attached to a molecule (protein or lipid) were taken into account, by scaling the calibration $\left(M_{\text {ref }}\right)$ with a fluorescence intensity ratio $F$ of a bulk, equimolar, detergent solutions of labelled species:

$$
F=\frac{I_{\mathrm{dp}}}{I_{\mathrm{dl}}}
$$

where $I_{\mathrm{dp}}$ and $I_{\mathrm{dl}}$ are fluorescence intensities of detergent solutions of labelled protein and lipid, respectively.

Membrane fluorescence peak intensities were calculated using a self-written macro (Section 2.5.5 and Script 1). 


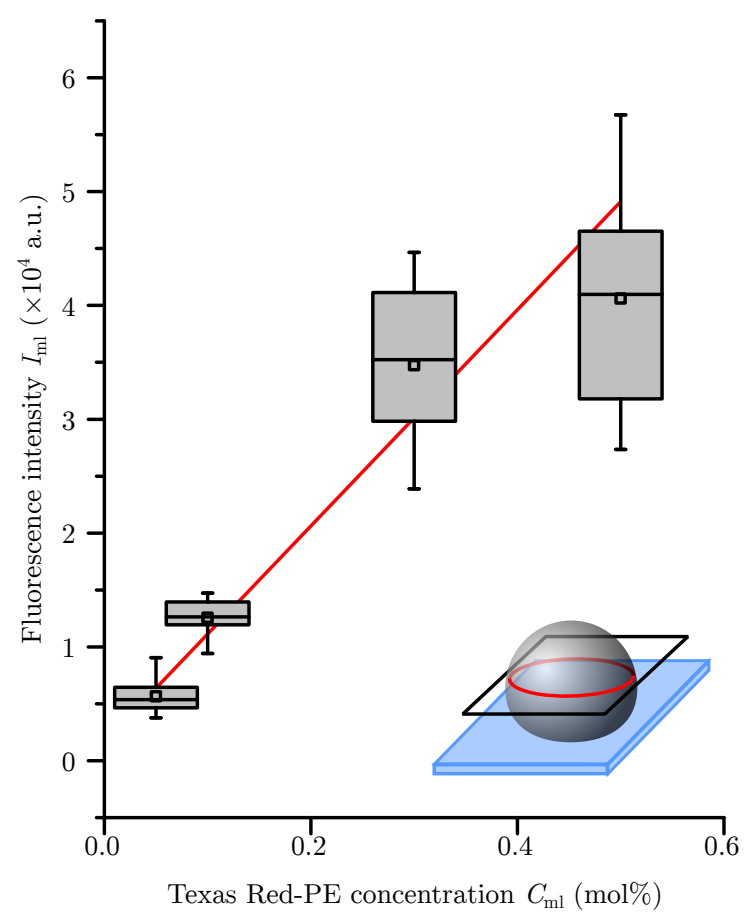

Figure 2.9. Calibration curve for determination of protein reconstitution efficiency into GUVs. Calibration curve (red line) for the acceptor complex membrane intensity determination, created by measuring membrane intensities for various TR-PE concentrations in GUVs (box plots, description as in Figure 3.9; $n=15,17,23$, and 14 starting from $0.05 \mathrm{~mol} \%$ TR-PE, respectively). Inset shows an equatorial focus plane used for membrane intensity determination. Figure and figure legend adapted from Witkowska and Jahn (2016).

\subsubsection{Diffusion coefficient determination of molecules incorporated into GUV membrane}

For calculation of lipid and protein diffusion coefficients in a GUV membrane, fluorescence recovery after photobleaching (FRAP) experiments were performed (section adapted from Witkowska and Jahn, 2016). FRAP is a traditional method to determine diffusion coefficient of molecules in the membrane (Poo and Cone, 1973, 1974). In this method, based on the diffusion speed of labelled species into the bleach spot from unbleached regions, the diffusion coefficient is determined. A circular bleach area was located on the top of the GUV (Figure 2.10 A, as in Tareste et al., 2008; Lira et al., 2014; Motta et al., 2015; Pincet et al., 2016). Digitised fluorescence intensity data were normalised and analysed with a self-written macro (Script 2 and 3) implementing data extraction from microscopy images in Fiji and automatic analysis in GNU Octave (Eaton et al., 2015), based on the manual by Miura (2012). Normalised data points were averaged for each group of GUVs with the same bleaching settings and coming 
A

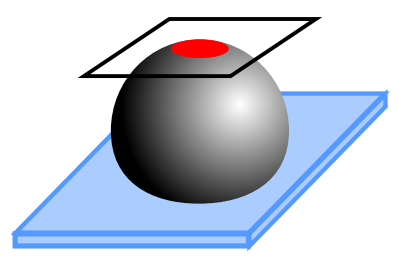

B

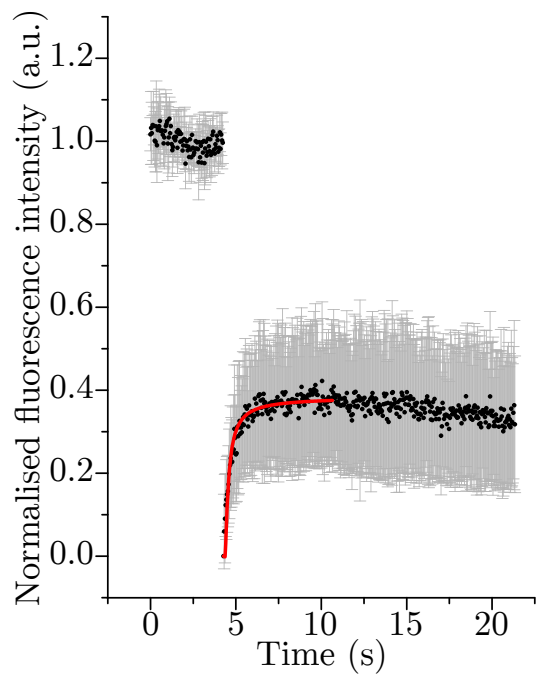

Figure 2.10. Diffusion coefficient determination of molecules incorporated into GUV membrane. (A) Schematic representation of bleach area at the top of the immobilised GUV used for the FRAP experiments. (B) Representative example of a mean FRAP recovery curve (black dots, grey error bars represent SD, $n=9$ ) for TR-PE used for diffusion coefficient determination (Figure 3.6) with a Soumpasis model (fit shown with a red line). Figure and figure legend adapted from Witkowska and Jahn (2016).

from the same preparation, and fitted to the formula developed by Soumpasis (1983):

$$
f(t)=A e^{\frac{-\tau}{2 t}}\left[I_{0}\left(\frac{\tau}{2 t}\right)+I_{1}\left(\frac{\tau}{2 t}\right)\right]
$$

where $I_{0}$ is the modified Bessel function of the first kind of order 0 and $I_{1}$ is the modified Bessel function of the first kind of first order, $A$ is the amplitude, $t$ is time and $\tau$ is the time constant. The diffusion coefficient $D$ is calculated from:

$$
D=\frac{w^{2}}{\tau}
$$

with $w$ being the radius of the bleached spot.

\subsubsection{Detection of fusion by lipid mixing}

The most traditional way to study vesicle fusion in vitro is by monitoring mixing of the lipids coming from the two populations of fusing vesicles (see also Section 1.3.2). 
Such lipid-mixing assays relay on the FRET (Förster, 1948) between fluorophores incorporated into vesicle membranes. The FRET signal (depending on the average distance between fluorophores) can either increase (i, Gibson and Loew, 1979) or decrease (ii, Struck et al., 1981; Weber et al., 1998) upon fusion, depending whether the FRET donor and FRET acceptor are localised on the membrane of the same initial set of vesicles. In the first case (i), the fluorophores are initially located on separate vesicles and only upon fusion-mediated lipid mixing, the fluorophores can perform the energy transfer from donor to acceptor (decrease in the donor fluorescence intensity, e.g. Gibson and Loew, 1979). In the second case (ii), both fluorophores are located on the same membrane at the beginning of the reaction, and fusion leads to incorporation of more unlabelled lipids to the membrane leading therefore to increase of the average distance between donor and acceptor fluorophore and decreased FRET (increase in the donor fluorescence intensity can be monitored, e.g. Struck et al., 1981). For the lipid mixing measurement performed with chromaffin granules and liposomes in Figure 2.4 donor dequenching was used (ii), while for the measurement of bulk lipid mixing observed under the microscope (Figure 3.10) donor quenching was used (i).

Bulk lipid mixing experiments (Figure 3.10; method description adapted from Witkowska and Jahn, 2016) involved GUVs containing 1.5 mol\% NBD-PE and LUVs containing $1.5 \mathrm{~mol} \%$ Rhodamine-PE. Upon vesicle fusion, FRET between these two labels occurs and thus NBD fluorescence is quenched. Based on the principle of acceptor photobleaching FRET microscopy (Bastiaens and Jovin, 1998; Jares-Erijman and Jovin, 2003), photobleaching of Rhodamine (FRET acceptor) in the equatorial plane of the GUV led to the recovery of NBD fluorescence.

In case of single vesicle to GUV fusion a non-FRET pair of fluorescence membrane labels was used (usually $\mathrm{DiO}$ and $\mathrm{DiD}$ ), and fusion was identified by the diffusion of LUV label in the GUV membrane. Images were acquired as $z$-stacks (containing usually 3-4 planes separated by $0.3 \mu \mathrm{m}$ ) over time in order to capture events where vesicle docking was followed by diffusion on GUV membrane (in 3 dimensions). Such $x y z t$ image stacks were then treated as continuous time sequences (sometimes processed additionally with a bicubic extrapolation algorithm) and were searched for docking, undocking, and fusion events. Fusion was additionally confirmed with membrane fluorescence intensity profiles (described in Section 2.5.5).

In order to describe chromaffin granule fusion dynamics, a cumulative probability distribution plot was fitted with a first-order kinetic equation as in Kiessling et al. (2013): 


$$
P(t)=A\left(1-e^{-t / \tau}\right)
$$

where $P$ is the value of cumulative probability of fusion after $t$ milliseconds from docking, $A$ is the amplitude, and $\tau$ is the time constant.

\subsubsection{Detection of fusion by content mixing}

For accurate description of membrane fusion, an evidence for mixing of the contents of two fusing vesicles is needed, in order to exclude leaky fusion, or that only hemifusion has happened (see also Section 1.3.2). The so called "content mixing" or "content release" assays have various forms, and can employ an enzymatic reaction (enzyme in one set of vesicles and substrate in the other, e.g. Ingolia and Koshland, 1978), formation of a chelation complex (e.g. Wilschut et al., 1980), or FRET with the use of dual labelled DNA hairpin (that would bind to unlabelled DNA coming from the second set of vesicles, e.g. Nickel et al., 1999; Diao et al., 2010). Here a dye dequenching principle was used that utilises calcein (Bowen et al., 2004) and sulforhodamine B (Kyoung et al., 2011).

In content mixing experiments syb-SUV lipid mix was dissolved in the liposome buffer (with cholate) in the presence of self-quenching concentrations of calcein $(200 \mathrm{mM}$, additionally protein to lipid ratio 1:200, Figure 3.11) or sulforhodamine B (100 mM, Figure 3.15). Release of the SUV content into the GUV lumen leads to rapid dilution of the content dye and increase in the fluorescence intensity. For the quantification of bulk content mixing (Figure 3.11), an average fluorescence intensity from a circular region representing GUV lumen (background subtraction) was calculated. In the case of the single vesicle fusion events, images were extrapolated with a bicubic algorithm and manually searched for fluorescence burst events occurring underneath the GUV membrane (Figure 3.15).

\subsubsection{Tracking of docked vesicles}

For vesicle tracking, very diluted and fluorescently labelled LUVs were added to immobilised $\Delta \mathrm{N}-\mathrm{GUVs}$ (labelled with another fluorescent dye) in order to obtain one docked vesicle per GUV. After identification of a GUV with a single docked LUV, a 3D stack was acquired over time ( $x y z t$ stack) with a fast confocal microscope (Leica SP8, 
A

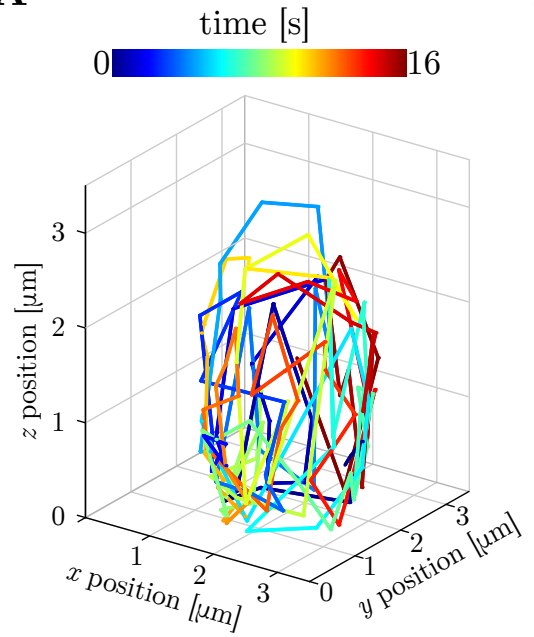

B

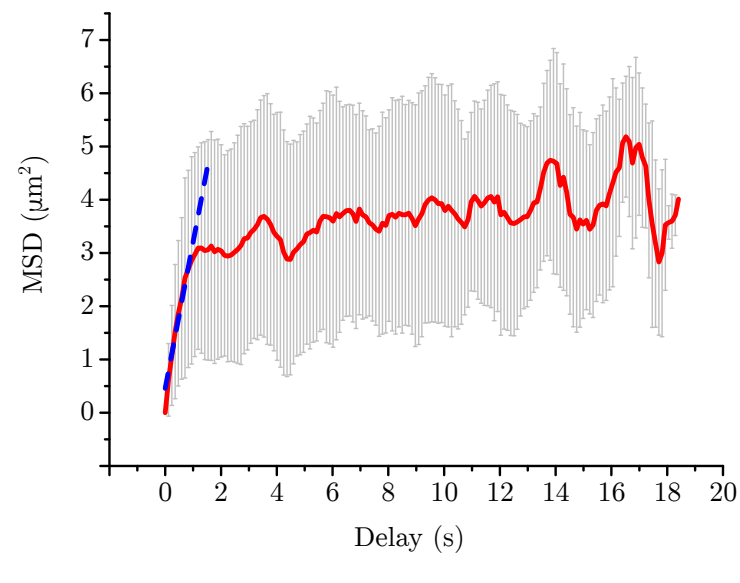

Figure 2.11. Analysis of vesicle diffusion on GUV surface by fluorescence microscopy. (A) An example trajectory obtained after tracking of a AA syb-LUV docked on a $\triangle \mathrm{N}-\mathrm{GUV}$ membrane with TrackMate. Track colour indicates time, temporal colour code bar shown above the graph. (B) An example MSD curve (red line) presenting mobility of a $\Delta 84$ syb-LUV on $\Delta$ N-GUV surface and fit (broken blue line, $\left.r^{2}=0.998\right)$ to the first linear part. Grey error bars represent SD.

Table A.7). Tracking analysis of fluorescently labelled docked vesicles (Figure 3.20) was performed with a TrackMate plugin for Fiji (LoG spot detector and Simple LAP tracker were utilised). Tracks after detection were manually curated, exported, and further analysed in MATLAB (MathWorks) with the @msdanalyser tool (Tarantino et al., 2014). From obtained trajectories (example trajectory shown in Figure 2.11 A) a mean square displacement (MSD) was calculated (Equation 12, example MSD plot shown in Figure 2.11 B):

$$
\operatorname{MSD}(\Delta t)=\frac{1}{T} \sum_{i=1}^{T}\left[r_{i}(t+\Delta t)+r_{i}(t)\right]^{2}
$$

where $\Delta t$ is delay, $T$ is the number of time points in a track, $r$ is displacement, and $t$ is time. The diffusion coefficient was calculated by fitting the first, linear part of the MSD curve (blue dashed line in Figure $2.11 \mathrm{~B}$ ) to the equation:

$$
\operatorname{MSD}(\Delta t)=2 d D \Delta t
$$

where $d$ is dimensionality of a problem (in our case $d=3$ for $3 \mathrm{D}$ data), and $D$ is the diffusion coefficient. 
Detection of vesicles with iSCAT microscopy (Section 2.5.2) was done with tools developed in the laboratory of Prof. Vahid Sandoghdar (Max Planck Institute for the Science of Light in Erlangen, Germany) and was done as described in Section 3.3.2, essentially according to Hsieh et al. (2014). Tracks were analysed by calculating the cumulative probability distribution $P$ of square displacements $r^{2}$ during delay $\Delta t$ (Figure 2.12; reason for using this method instead of MSD described in Hsieh et al., 2014), and fitting it to one $\left(\varepsilon_{2}=0\right)$ or two $\left(\varepsilon_{2} \neq 0\right)$ component distribution function (depending on the residual fitting error determining fit quality; Hsieh et al., 2014) in order to extract diffusion coefficients:

$$
P\left(r^{2}, \Delta t\right)=1-\varepsilon_{1} e^{-r^{2} / 4 D_{1} \Delta t}-\varepsilon_{2} e^{-r 2 / 4 D_{2} \Delta t}
$$

where $D_{1}$ and $D_{2}$ are the major and minor diffusion coefficients, respectively; and $\varepsilon_{1}$ and $\varepsilon_{2}$ are their corresponding weighting factors $\left(\varepsilon_{1}+\varepsilon_{2}=1\right.$, with $\left.\varepsilon_{1}>\varepsilon_{2}\right)$.

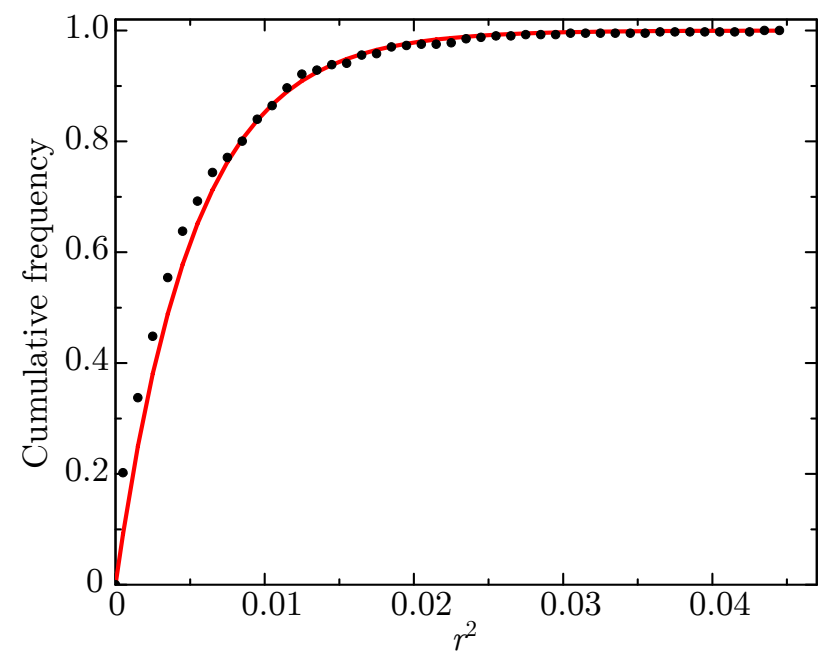

Figure 2.12. Diffusion coefficient determination from an iSCAT trajectory. Example plot of a cumulative probability of squared displacement $-P\left(r^{2}\right)$ analysis of an iSCAT trajectory of a syb-LUV docked on the GUV membrane, used for determination of the diffusion coefficient. Black dots represent experimental data and red line an exponential fit to a one component equation. 



\section{Results}

\subsection{Optimisation and characterisation of $\Delta \mathrm{N}-\mathrm{GUVs}$}

For the development of a new fusion assay involving GUVs for studying SNAREmediated membrane fusion, it was necessary to optimise a formation protocol of GUVs containing SNARE proteins and characterise these GUVs' properties.

\subsubsection{Protocol development of $\Delta \mathrm{N}-\mathrm{GUV}$ formation}

Earlier, neuronal SNARE proteins were reconstituted into GUVs with a proteoliposome drying method with electroformation (Bacia et al., 2004; Tareste et al., 2008; Hui et al., 2009; Malsam et al., 2012), an inkjet method (Richmond et al., 2011), or more recently (published after I have established GUV formation protocol) an osmotic shock method (Motta et al., 2015). Here, in order to study SNARE-mediated exocytosis, an artificial SNARE acceptor complex ( $\Delta \mathrm{N}$ complex) was used that was previously shown to speed up liposome fusion (Pobbati et al., 2006). Initially, I carried out the GUV formation starting from $\Delta \mathrm{N}-\mathrm{LUVs}$ dried in the liposome buffer on the surface of ITO-slides with rehydration in water and a short electroformation protocol (1.5 h, see Table 3.1), but the yield of GUVs was not so high and vesicles were rather small. For this reason, I tested multiple protocols to improve $\Delta \mathrm{N}-\mathrm{GUV}$ quality and possibly avoid loss of complex activity due to drying and/or no-salt conditions (for an overview of experimentally tested ranges of parameters see Table 3.1).

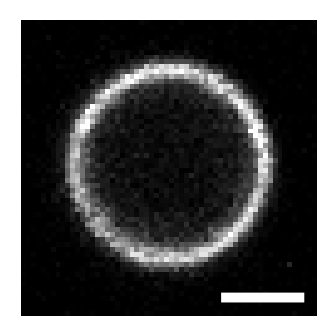

Figure 3.1. Image of a $\Delta \mathrm{N}-\mathrm{GUV}$. Confocal micrograph presenting an example of a $\Delta$ N-GUV prepared with an optimised GUV formation protocol (Table 3.1) taken at the equatorial plane of the vesicle. Fluorescence comes from the $\Delta \mathrm{N}$ complex that is labelled with TR. Scale bar $2 \mu \mathrm{m}$. 
Table 3.1. Parameters of various conditions tested during protocol optimisation for $\Delta$ N-GUV formation. Listed parameters are grouped in columns indicating subsequent steps of GUV formation procedure. Black indicates set of parameters chosen for further experiments. Footnotes contain references to example publications utilising the same (or very similar) conditions. For details see also Section 2.3.3.

\begin{tabular}{|c|c|c|c|}
\hline Starting material & Lipid film formation & Rehydration buffer & Electroformation \\
\hline \multirow{5}{*}{$\begin{array}{l}\text { - } \Delta \text { N-LUVs }{ }^{a} \\
\text { - } \Delta \mathrm{N}-S U V s^{b} \\
\text { - Lipid mix in organic } \\
\text { solvent and purified } \\
\text { } \Delta \mathrm{N} \text { complex }{ }^{c}\end{array}$} & $\underline{\text { Buffer }}$ & $\underline{\mathrm{KCl} \text { concentration }}$ & \multirow{9}{*}{$\begin{array}{l}\text { - } V_{\mathrm{pp}}=1.2-5 \mathrm{~V}, 1 \mathrm{~h}, \\
10 \mathrm{~Hz}^{b}+30 \mathrm{~min}, 4 \mathrm{~Hz} \\
\text { - } V_{\mathrm{pp}}=3.4 \mathrm{~V}, 3 \mathrm{~h}, 10 \mathrm{~Hz}^{b} \\
+1 \mathrm{~h}, 4 \mathrm{~Hz} \\
\text { - "high salt protocols" g,i }\end{array}$} \\
\hline & - with $100-150 \mathrm{mM} \mathrm{KCl}^{b}$ & - $150 \mathrm{mM}^{g}$ & \\
\hline & - with 0-5 mM KCl & - $5 \mathrm{mM}^{g}$ & \\
\hline & - containing sucrose ${ }^{a}$ & - $\mathrm{no}^{b}$ & \\
\hline & - with detergents ${ }^{d}$ & $\underline{\text { Sucrose concentration }}$ & \\
\hline \multirow{4}{*}{$\begin{array}{l}\text { - Pure lipid mix and } \\
\text { protein reconstitution } \\
\text { after GUV formation }{ }^{d}\end{array}$} & $\underline{\text { Drying }}$ & - $1 \mathrm{M}$ & \\
\hline & - Vacuum $1-12 \mathrm{~h}^{b}$ & - $200-500 \mathrm{mM}^{b, g}$ & \\
\hline & - Room atmosphere 5 min-1 he & - $\mathrm{no}^{h}$ & \\
\hline & - Saturated salt atmosphere ${ }^{f}$ & & \\
\hline
\end{tabular}

${ }^{a}$ Doeven et al., 2005

${ }^{b}$ SNARE reconstitution into GUVs in Bacia et al., 2004

${ }^{c}$ van den Bogaart et al., 2011; Nikolaus et al., 2010

${ }^{d}$ see description on the next page and Dezi et al., 2013

'Motta et al., 2015

${ }^{f}$ Girard et al., 2004

${ }^{g}$ Aimon et al., 2011

${ }^{h}$ Angelova et al., 1992

${ }^{i}$ Pott et al., 2008 
Regarding protein reconstitution into GUVs, there is a previously published report about detergent-mediated reconstitution of membrane proteins into GUVs after GUVs are already formed (Dezi et al., 2013). Approach presented by Dezi et al. has three major advantages: (i) protein or protein complex is not dried and is maintained in detergent solution before membrane incorporation, (ii) GUVs are formed before protein incorporation which allows formation of much larger vesicles, and (iii) presence of detergents allows relatively easy equilibration of buffers between the outside and the lumen of the GUV and therefore allows buffer change to a more physiologically relevant one (i.e. containing salt and of controlled pH). In this method GUVs are grown in the presence of mild detergents, like DOTM (n-Dodecyl- $\beta$-D-thiomaltopyranoside, at concentration slightly above the critical micelle concentration - CMC), and purified membrane protein in detergent solution is subsequently reconstituted into the preformed GUVs. Finally, the detergent is removed by incubation with adsorbent beads (e.g. Bio-Beads). During the course of my work, I tested multiple sugar-based detergents in order to reconstitute the $\Delta \mathrm{N}$ complex directly into preformed GUVs (see Table A.2 for the list of tested detergents). GUVs were formed in the presence of NTG, DG, and DOTM, with sufficient yield only in case of the last one. However, upon addition of purified and fluorescently labelled $\Delta \mathrm{N}$ complex, no sufficient GUV membrane incorporation was detected. It needs to be mentioned that only one concentration was tested for each detergent.

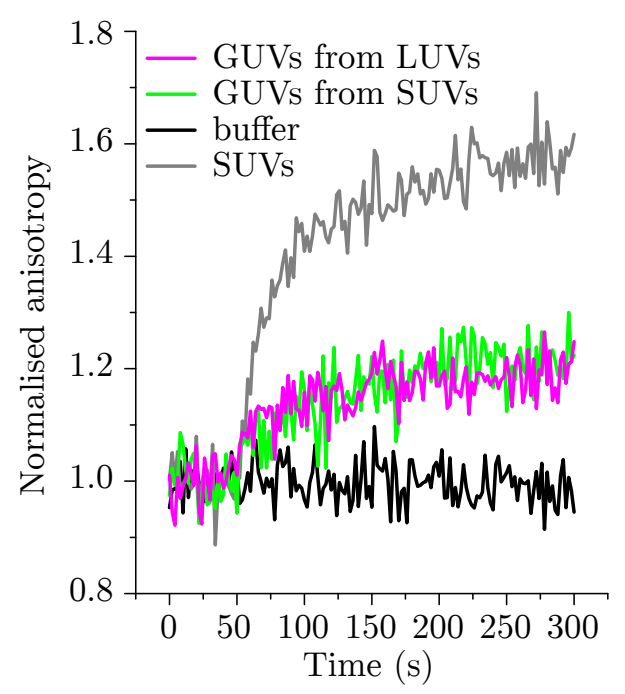

Figure 3.2. Comparison of $\Delta \mathrm{N}$ complex activity in GUVs prepared from SUVs or LUVs. Binding of syb 1-96 labelled with OG488 to the $\Delta \mathrm{N}$ complexes incorporated into GUV membrane measured as bulk anisotropy change. GUVs are prepared either from $\Delta \mathrm{N}-\mathrm{LUVs}$ (magenta) or $\Delta \mathrm{N}-\mathrm{SUV}$ s (green). Controls present addition of equivalent volume of buffer (black) or equivalent concentration of $\Delta \mathrm{N}-\mathrm{SUVs}$ (grey line) instead of GUVs. 
Other parameters tested to avoid drying-mediated protein degradation were liposome equilibration in a no-salt buffer prior to drying, or prevention of crystal formation by addition of sucrose (Table 3.1; Doeven et al., 2005; Crowe et al., 1988). In this case, no noticeable difference, neither in GUV morphology nor in protein activity, was detected in comparison to drying in the presence of liposome buffer without sucrose. In order to further improve GUV formation yield, parameters like drying (under vacuum, in saturated salt atmosphere, or in room atmosphere), sucrose concentration in the rehydration buffer, and electroformation protocol length were tested. The best quality of GUVs and highest complex activity were obtained from proteoliposomes in the liposome buffer, dried in an exsiccator overnight, and subsequently rehydrated with $200 \mathrm{mM}$ sucrose solution in water, followed by a $4 \mathrm{~h}$ electroformation protocol (see black marking in Table 3.1 and Figure 3.1). Neither GUV morphology (data not shown) nor $\Delta \mathrm{N}$ complex activity were dependent on the liposome type - SUVs or LUVs - used as a starting material (Figure 3.2).

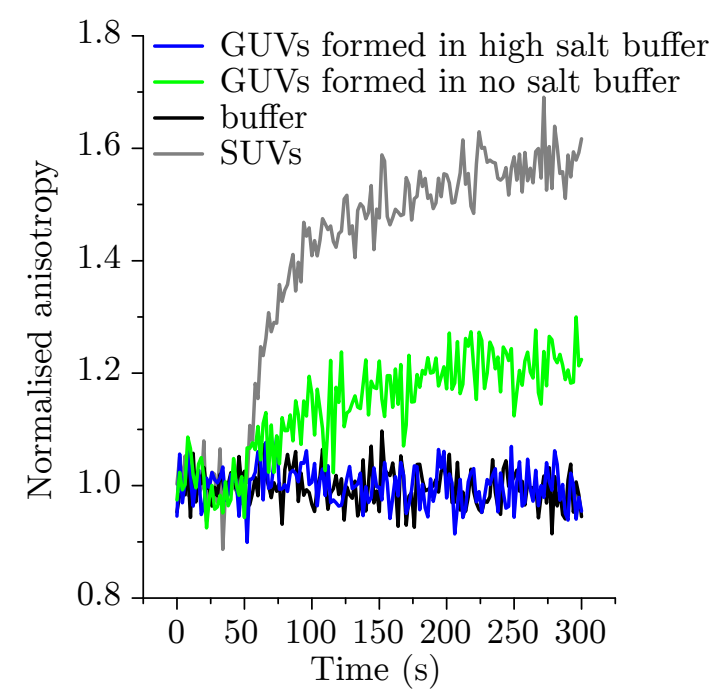

Figure 3.3. Comparison of $\Delta \mathrm{N}$ complex activity in GUVs prepared by electroformation in high or no salt buffer. Bulk anisotropy measurement as in Figure 3.2. GUVs prepared from $\Delta \mathrm{N}-\mathrm{SUVs}$ and electroformed with different protocols in buffers with $(150 \mathrm{mM} \mathrm{KCl}$, blue line) or without (green line) salt. Controls present addition of equivalent volume of buffer (black line) or equivalent concentration of $\triangle \mathrm{N}-\mathrm{SUVs}$ (grey line) instead of GUVs. All data except blue line the same as in Figure 3.2.

Finally, I tested protocols for GUV formation in physiologically relevant buffers that would maintain the salinity and $\mathrm{pH}$ of the buffer throughout the GUV preparation procedure. Protocols published before that reported successful formation of GUVs containing active membrane protein (Pott et al., 2008; Aimon et al., 2011) indicate 
that electroformation with buffers of higher salinity (from $100 \mathrm{mM} \mathrm{NaCl}$ or $\mathrm{KCl}$ ) has to be performed at higher formation frequencies $(500 \mathrm{~Hz}$ in comparison to $10 \mathrm{~Hz}$ used in no-salt protocols). In a protocol presented in Aimon et al. (2011) electroformation is performed overnight at relatively low voltage $\left(V_{\mathrm{pp}}=0.85 \mathrm{~V}\right)$, while in the other report by Pott et al. (2008) protocol is shorter (around $3 \mathrm{~h}$ ) with higher voltage $\left(V_{\mathrm{pp}} \approx 2.8 \mathrm{~V}\right.$ ). Using these protocols with ITO-slides led to relatively low GUV yield and, what is more, the $\Delta \mathrm{N}$ complex after such a procedure was inactive as confirmed by syb 1-96 binding test (Figure 3.3).

\subsubsection{Morphological and biochemical characterisation of $\Delta N-G U V s$}

GUVs formed with an optimised protocol (see Section 3.1.1) were characterised with regards to their morphology (diameter and lamellarity), as well as to the efficiency and quality of the $\Delta \mathrm{N}$ complex incorporation.

A

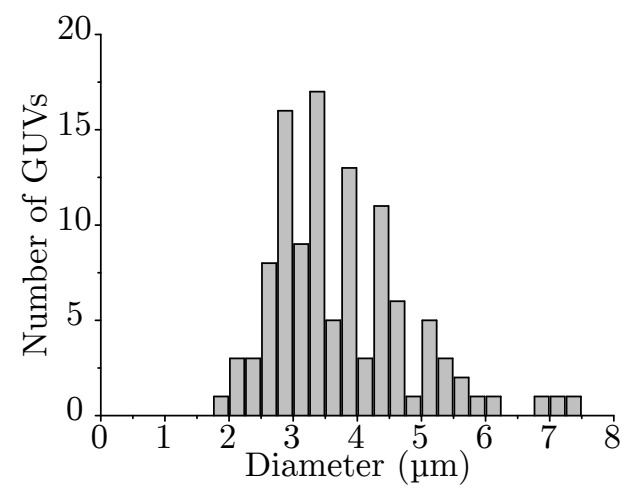

B

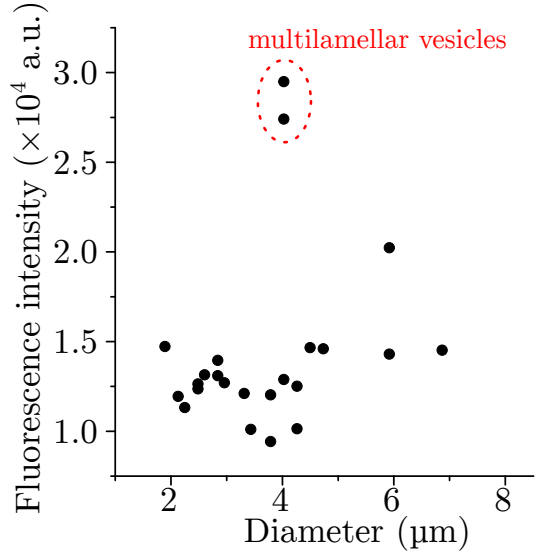

Figure 3.4. Distribution of $\Delta \mathrm{N}-$ GUVs diameter and lamellarity. (A) Histogram presenting size distribution of GUVs formed with an optimised protocol (for details see Section 3.1.1). Presented 111 GUVs, bin $=0.25 \mu \mathrm{m}$. (B) Representative scatter plot presenting peak membrane fluorescence intensity of $\Delta \mathrm{N}-\mathrm{GUVs}$ containing $0.1 \mathrm{~mol} \%$ of TR-PE against their diameter. In red marked vesicles that are probably multilamellar. Presented total of 23 vesicles that were not manually preselected for the membrane brightness.

In order to measure GUV diameters confocal images were acquired at the equatorial plane of fluorescently labelled vesicles immobilised in the imaging chambers. Then, a circular selection was fitted to the GUV structure and diameter was measured. GUVs formed with the protocol presented in this thesis were relatively small (on average 
$3.75 \pm 0.10 \mu \mathrm{m}$ in diameter, Figure $3.4 \mathrm{~A}$ ) but big enough to be suitable for microscopy imaging. Further, GUVs were analysed with regards to their lamellarity. Electroformation with ITO slides is known to produce mainly unilamellar vesicles but presence of protein in the preparation clearly influences the formation mechanism affecting for example vesicle size (GUVs prepared without proteins with the same protocols had often diameters $>10 \mu \mathrm{m}$, data not shown). Thus, when optimising a proteo-GUV formation protocol it is necessary to determine vesicle lamellarity. Since membrane bilayer thickness is below the microscope resolution, the most standard method for lamellarity determination has been measurement of membrane fluorescence intensity and plotting it against the vesicle diameter (Akashi et al., 1996; Chiba et al., 2014). More recently, methods based on differential interference contrast microscopy were developed (McPhee et al., 2013). In this study, a method involving measurement of membrane fluorescence intensity was utilised. An example graph presenting peak membrane fluorescence intensity (Section 2.5.5) of $\Delta \mathrm{N}$-GUVs containing $0.1 \mathrm{~mol} \%$ of TR-PE plotted against vesicle diameter is shown in Figure 3.4 B. Clearly only 2 vesicles exhibit fluorescence intensities that are $\sim 2$ times higher than other vesicles of the same diameter, and thus, most probably are bi-lamellar. Therefore, it can be concluded that most of the vesicles prepared with the optimised protocol (Table 3.1) are unilamellar.

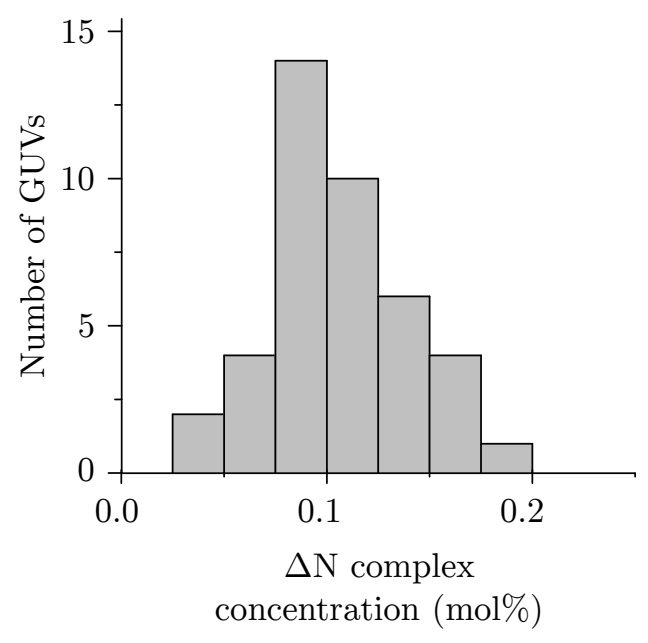

Figure 3.5. Determination of $\Delta \mathrm{N}$ complex concentration in a GUV membrane. Histogram showing the distribution of the concentration of the acceptor complex in the membrane of GUVs $(n=41$, bin size $=0.025 \mathrm{~mol} \%)$ obtained by comparing membrane peak fluorescence intensity at the vesicle equator of GUVs containing labelled $\Delta \mathrm{N}$ complex with GUVs containing known concentration of labelled lipid (TRPE, for details see Section 2.5.6). Figure and figure legend adapted from Witkowska and Jahn (2016). 
Next, in order to determine the incorporation efficiency of $\Delta \mathrm{N}$ complex into GUVs, the method initially developed by Galush et al. (2008) and adapted for GUVs by Aimon et al. (2011) was used. In this method, membrane fluorescence intensities are compared between fluorescently labelled lipids, incorporated at known concentrations, with fluorescently labelled protein, reconstituted with a certain GUV formation protocol (for details see Section 2.5.6). For this purpose, membrane intensities of $\Delta N-G U V s$ containing a range of TR-PE concentrations were measured, along with GUVs containing TR- $\Delta \mathrm{N}$ complex (Figure 2.9), and membrane concentration of $\Delta \mathrm{N}$-complex in a number of GUVs was calculated (Figure 3.5). Small unilamellar liposomes used for preparation of $\Delta \mathrm{N}-\mathrm{GUVs}$ were formed at protein to lipid ratio of $1: 1,000$, so the theoretical concentration at $100 \%$ incorporation efficiency would be $0.1 \mathrm{~mol} \%$. Distribution of protein membrane concentration presented in Figure 3.5, with mean value of $0.106 \pm 0.005 \mathrm{~mol} \%$ suggests that there is no change in protein and lipid composition during GUV formation protocol due to e.g. protein precipitation.

A

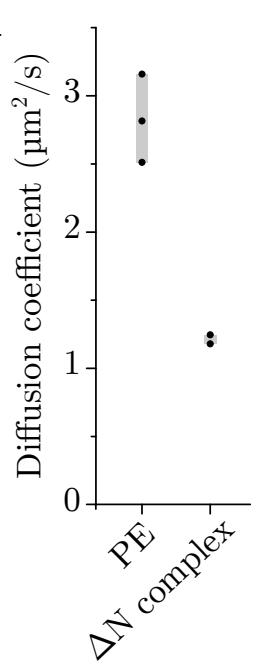

B

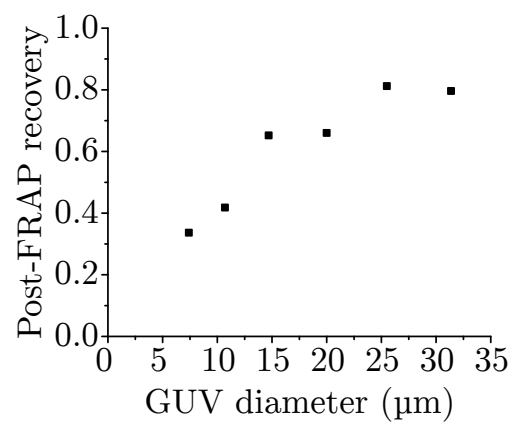

Figure 3.6. Determination of protein and lipid mobility in $\Delta$ N-GUVs. (A) Mobility of the SNARE acceptor complex in the plane of the GUV membrane in comparison to the mobility of membrane lipids. The diffusion coefficients were determined by performing FRAP measurements of TR-PE or TR- $\Delta \mathrm{N}$ complex using bleaching of an area on the top of the GUVs. Dots represent mobilities obtained for groups of GUVs and grey bars represent range of obtained values $\left(n_{\mathrm{PE} \text { total }}=19\right.$, $n_{\Delta \mathrm{N} \text { total }}=16$ ). Panel A (figure and figure legend) adapted from Witkowska and Jahn (2016). (B) Graph presenting dependency of the amplitude of post-FRAP recovery on the GUV diameter. Presented data from 6 GUVs.

The next step was to check mobility of species (proteins and lipids) in GUVs. For this purpose, FRAP measurements were performed with $\Delta \mathrm{N}-\mathrm{GUVs}$ containing either fluorescently labelled lipid (TR-PE) or protein (TR- $\Delta \mathrm{N}$ complex). The fraction of 
fluorescently labelled species was bleached on top of the GUV (Figure $2.10 \mathrm{~A}$ ), and the subsequent diffusion-based recovery of the fluorescence intensity was monitored (Figure $2.10 \mathrm{~B}$ ). From the kinetics of the fluorescence recovery, diffusion coefficients for $\mathrm{PE}$ and $\Delta \mathrm{N}$ complex were determined (Figure $3.6 \mathrm{~A}$ ) to be in the range of $2.5-3.2 \mu \mathrm{m}^{2} / \mathrm{s}$ and $\sim 1.2 \mathrm{\mu m}^{2} / \mathrm{s}$, respectively. Since there were quite low recovery values (around 30-40\%, see example recovery curve in panel B Figure 2.10), protein-free GUVs that can have much larger diameters, were tested for the dependency of the post-FRAP recovery amplitude on GUV diameter (Figure $3.6 \mathrm{~B}$ ). This experiment showed that GUVs with smaller diameters (similar to diameters of $\Delta \mathrm{N}-\mathrm{GUVs}$ ) have relatively low recovery amplitudes, while larger the GUV, higher the amplitude; up to around $25 \mu \mathrm{m}$ of diameter where recovery reaches values of above $80 \%$ (Figure $3.6 \mathrm{~B}$ ). This result may suggest that low post-FRAP recovery values in $\Delta \mathrm{N}-\mathrm{GUVs}$ are not due to the fraction of immobile species but rather the result of photobleaching of fairly large fraction of available fluorophores during the bleaching step of the FRAP experiment.

\subsubsection{Assessment of fusogenic activity of $\Delta \mathrm{N}-\mathrm{GUVs}$}
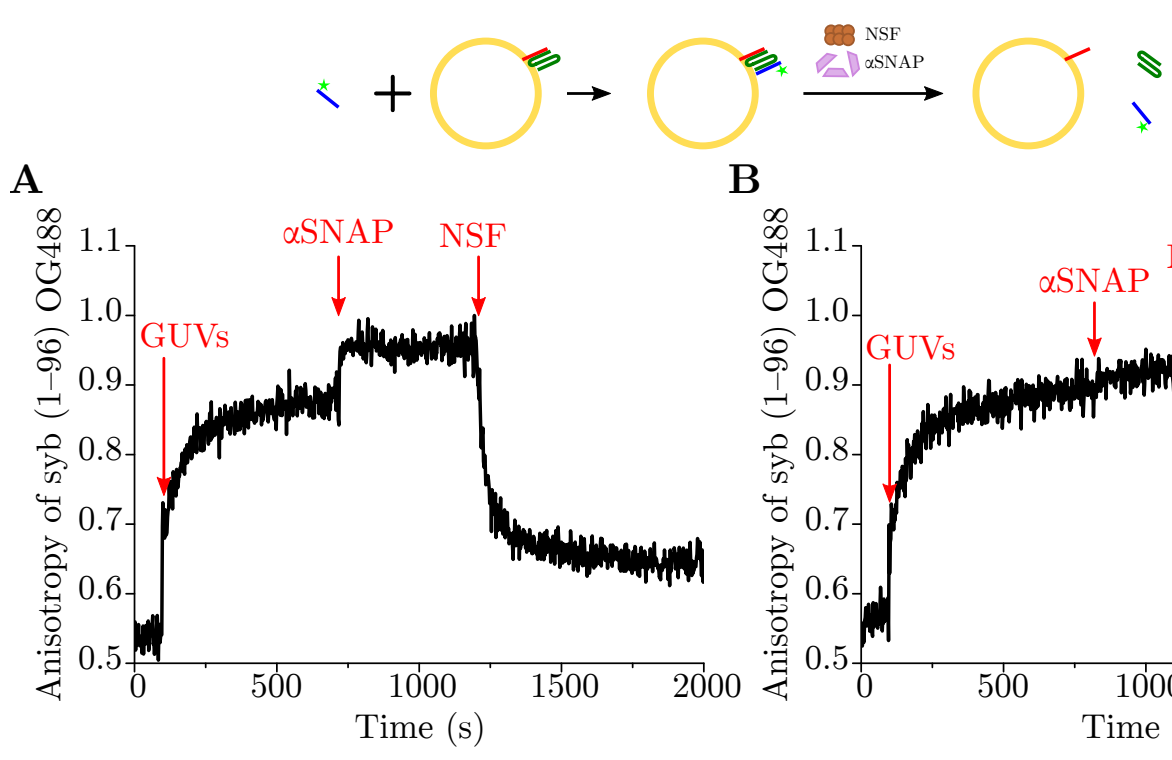

B

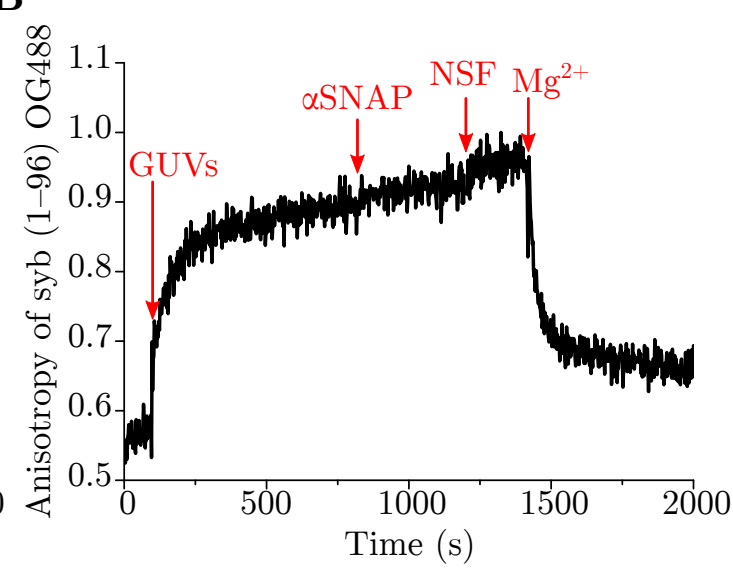

Figure 3.7. Assembly and disassembly of SNARE complexes on GUV membrane (monitored in solution with fluorescence anisotropy). Graphs presenting change of fluorescence anisotropy of OG488 attached to syb 1-96 in time. $\Delta \mathrm{N}-\mathrm{GUVs}$, purified $\alpha$ SNAP and purified NSF were added in sequence at the time points indicated with red arrows. Both reactions where the same except that disassembly buffer in reaction presented in panel $(\mathrm{B})$ did not contain $\mathrm{Mg}^{2+}$ but it was added at the later stage of the reaction indicated on a graph. 
A
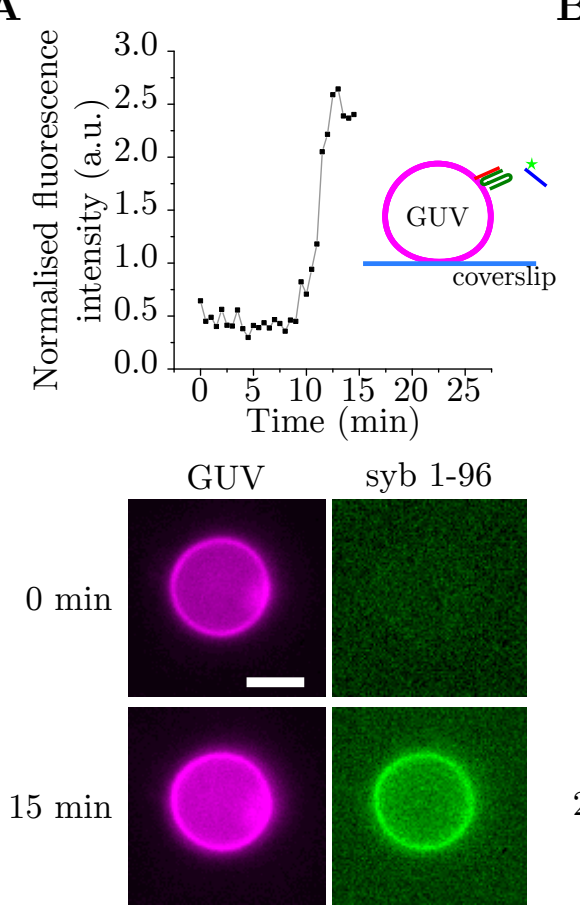

B


Figure 3.8. Assembly and disassembly of SNARE complexes on GUV membrane (monitored with microscopy). Measurement of OG488 fluorescence intensity change (coming from syb 1-96 labelled with OG488) on GUV membrane (labelled with Rho-PE), expressed as ratio of peak membrane fluorescence intensities of OG488 (background subtracted) and Rho. Graph presented in panel A shows increase of OG488 intensity on a membrane upon addition of syb 1-96 OG488, and graph in panel $\mathrm{B}$ subsequent decrease of intensity after addition of NSF and $\alpha$ SNAP. Images presented below graphs represent 1st and last measurement points from the graphs above. Scale bar $3 \mu \mathrm{m}$.

Next, $\Delta \mathrm{N}-\mathrm{GUV}$ s had to be tested to asses whether the reconstituted $\Delta \mathrm{N}$ complex is still fusion active. This complex was previously shown to induce fusion of small and large liposomes (see Pobbati et al. 2006 for SUVs and Hernandez et al. 2012 for LUVs). To test the ability of the $\Delta \mathrm{N}$ complex to form a ternary SNARE complex, binding of syb (1-96) to $\triangle \mathrm{N}-\mathrm{GUVs}$ was monitored with fluorescence anisotropy (Figure 3.7, similar to measurements presented in Figure 3.2 and 3.3). To confirm that anisotropy increase resulted from a SNARE complex assembly, a SNARE disassembly machinery ( $\alpha$ SNAP and NSF) was subsequently added to the reaction mixture. Anisotropy upon addition of $\alpha$ SNAP increased, suggesting binding of this protein to assembled SNARE complexes that further reduced the dye mobility. After addition of NSF, the complexes were disassembled, and thus anisotropy decreased again (panel A in Figure 3.7). This disassembly reaction was NSF specific because there was no anisotropy drop in case of $\mathrm{Mg}^{2+}$-free buffer that is necessary for ATP hydrolysis by NSF (compare anisotropy values before 
and after adding $\mathrm{Mg}^{2+}$ in panel B in Figure 3.7). The same assembly-disassembly reaction could also be observed with microscopy with $\Delta \mathrm{N}$-GUVs immobilised in imaging chamber with added syb 1-96 labelled with OG488 (Figure 3.8). Association of syb 1-96 with GUV membrane (panel A in Figure 3.8), subsequent NSF-mediated SNARE complex disassembly, and dissociation of soluble syb 1-96 (panel B in Figure 3.8) from the GUV membrane labelled with Rho-PE was monitored by determination of membrane-localised OG488 fluorescence intensity with time-lapse image acquisition. However, addition of disassembly buffer (see Table A.6) leads to GUV shape change so one has to be careful with interpretation of fluorescence intensity change data for NSF-mediated SNARE complex disassembly (see inset with images at time 0 min and 25 min in panel B Figure 3.8).

A

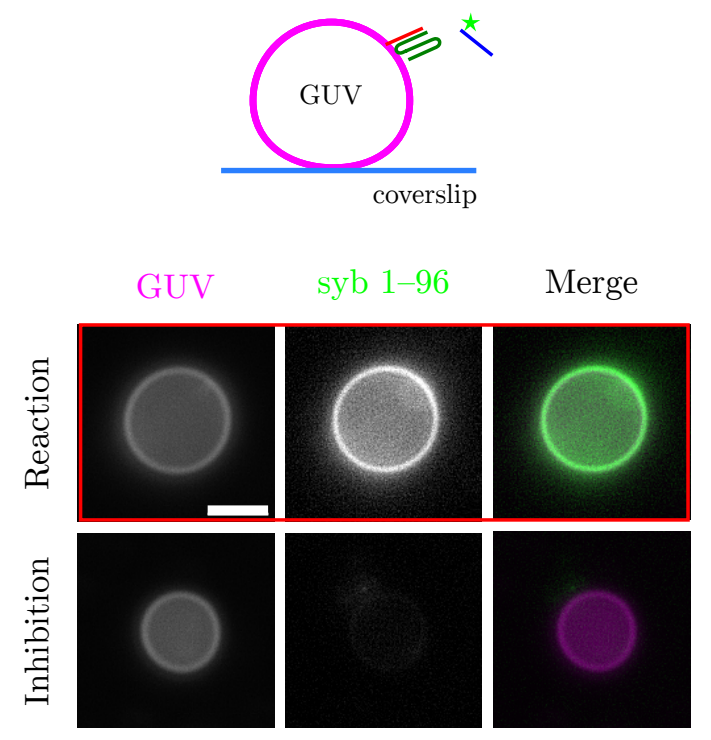

B



Figure 3.9. Ternary SNARE complex formation on $\Delta \mathrm{N}-\mathrm{GUV}$ membrane. (A) Microscopy images showing binding of a synaptobrevin fragment lacking its transmembrane domain (syb 1-96, labelled with the fluorescent dye OG488) to the GUV membrane containing acceptor complex (upper panel) or onto GUVs with acceptor complex preincubated with non-labelled syb 1-96 (lower panel). Scale bar $3 \mu \mathrm{m}$. (B) Quantification of a representative experiment assessing SNARE complex formation between syb 1-96 and $\Delta \mathrm{N}$ complex on GUV membrane. The box plot shows quantification of the membrane fluorescence intensity (background corrected) resulting from syb-OG attachment onto the GUV. Boxes represent interquartile range and whiskers below and above indicate full data range. Line in a box represents median and square point represents mean ( $n=9$ and 13 for reaction and inhibition, respectively). Figure and figure legend adapted from Witkowska and Jahn (2016). 
Binding of syb 1-96 OG488 to GUV membrane visualised with microscopy was then used routinely as a $\Delta \mathrm{N}$ complex activity test with a control where $\Delta \mathrm{N}-\mathrm{GUV}$ s are preincubated with an excess of unlabelled syb 1-96. Quantification of a representative experiment is shown in Figure 3.9.

A

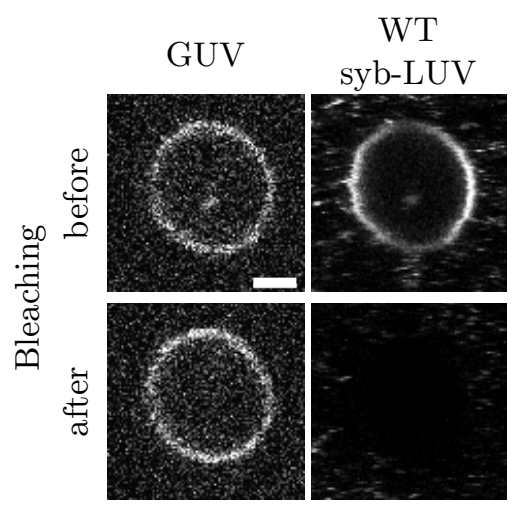

B
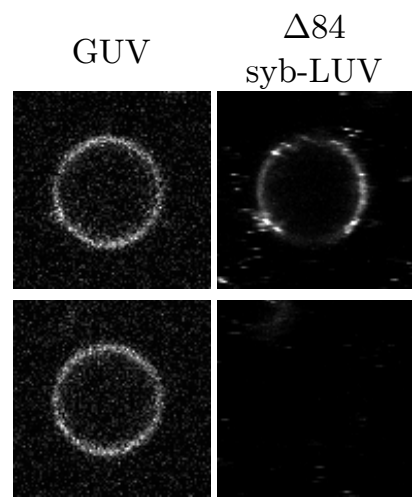

C
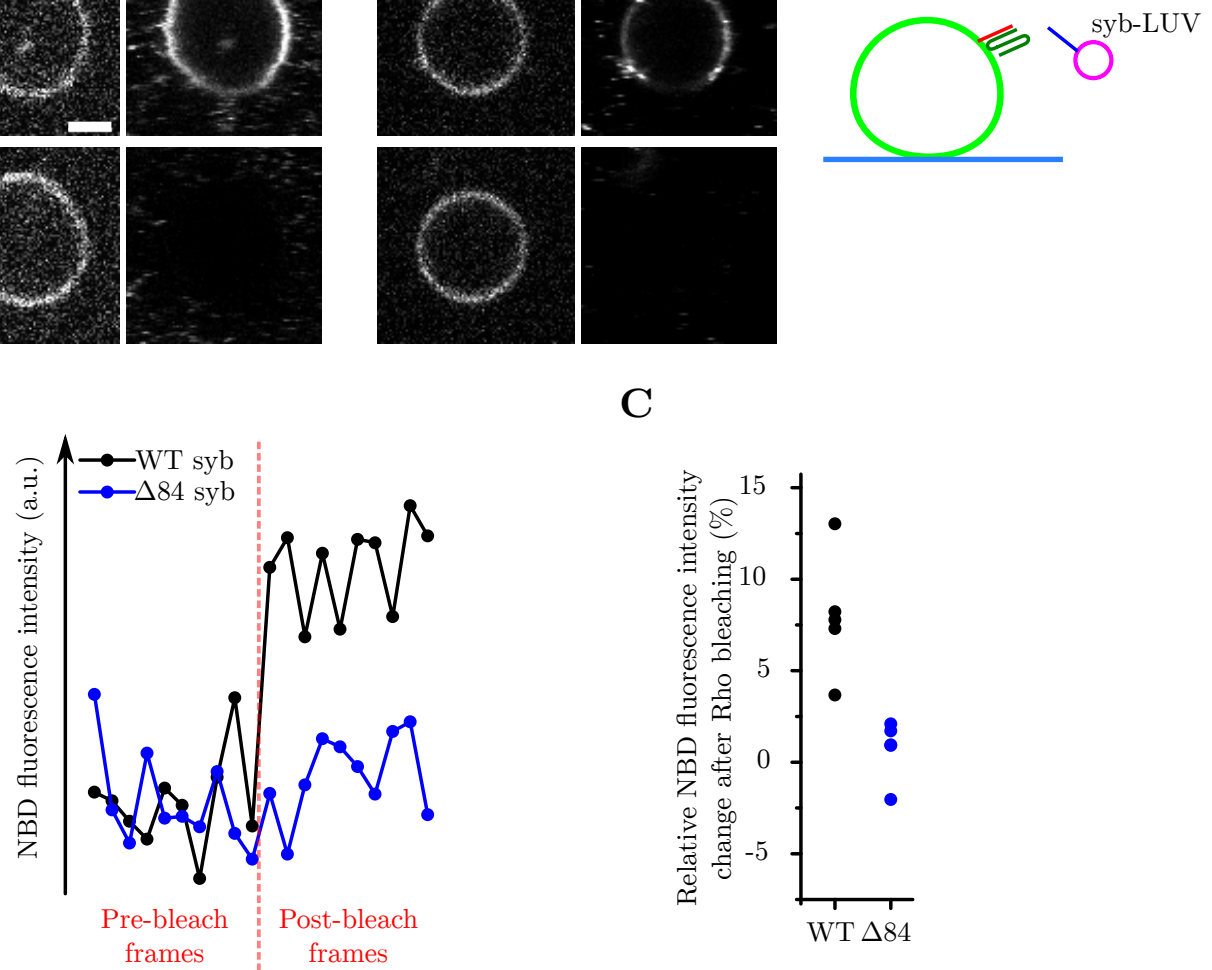

Figure 3.10. SNARE acceptor complex reconstituted into GUVs is able to induce lipid mixing between GUVs and syb-liposomes. (A) Microscopy images showing $\triangle \mathrm{N}-\mathrm{GUVs}$ containing NBD-PE after approximately 30 min incubation with LUVs labelled with Rho-PE containing either WT syb (right) or $\Delta 84$ syb (left) before or after rhodamine bleaching. Scale bar $2 \mu \mathrm{m}$. (B) Quantification of donor (NBD) fluorescence after acceptor (rhodamine) bleaching. Fluctuations of donor fluorescence in the GUV membrane before and after bleaching, shown in a representative experiment. (C) Change of the acceptor fluorescence intensity after the bleach (for each measurement, 10 data points were averaged before and after the bleach, $n=5$ for each group). Figure and figure legend adapted from Witkowska and Jahn (2016).

Finally, $\Delta \mathrm{N}-\mathrm{GUVs}$ were controlled for ability to fuse with liposomes reconstituted with synaptobrevin. For monitoring fusion, both a lipid mixing (Figure 3.10) and a content mixing assay were utilised (Figure 3.11). For a lipid mixing assay, a FRET-based approach was used where FRET donor fluorescent dye is incorporated in the membrane 
of a one fusing vesicle, and FRET acceptor in the membrane of a second fusing vesicle. In this case, $\Delta \mathrm{N}-\mathrm{GUV}$ s were labelled with NBD-PE - a FRET donor, and syb-LUVs with Rho-PE - a FRET acceptor. Upon fusion, membrane lipids, and thus fluorescent dyes, mix with each other and energy of the FRET donor is transferred to the acceptor resulting in the lowered FRET donor fluorescence emission. In order to detect FRET on a confocal microscopy setup, increase of donor fluorescence upon acceptor photobleaching was monitored. FRET was only observed when LUVs containing WT syb were used (black data points Figure $3.10 \mathrm{~B}$ ) and not when $\Delta 84 \mathrm{syb}$ - a syb mutant that was shown before to arrest large liposomes in a docked state (blue data points in Figure 3.10 B; Hernandez et al., 2012; see also Section 1.2.4). This result suggests that there is lipid mixing occurring between membranes of GUV and LUV induced by SNARE-mediated membrane fusion.

A

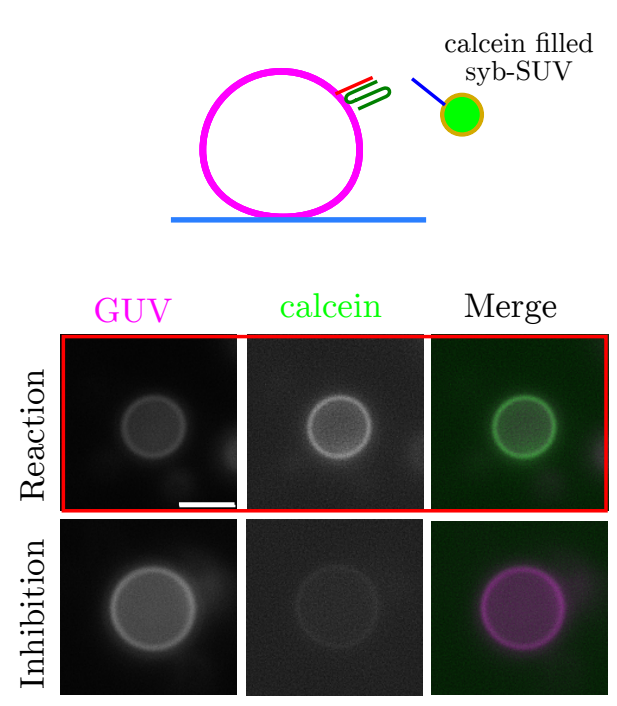

B

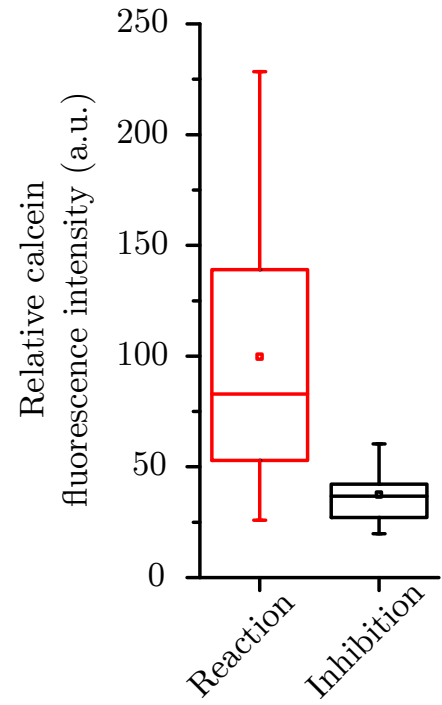

Figure 3.11. SNARE acceptor complex reconstituted into GUVs is able to induce content mixing between GUVs and syb-liposomes. (A) Microscopy images showing luminal calcein fluorescence of $\Delta \mathrm{N}-\mathrm{GUVs}$ after $30 \mathrm{~min}$ incubation in the presence of syb-SUVs filled with calcein. GUVs shown in the lower panel were preincubated with non-labelled syb 1-96 (lower panel). Scale bar 3um. (B) Quantification from a representative experiment of a GUV luminal fluorescence intensity (relative values, corrected for background fluorescence) resulting from fusion with calcein filled SUVs (for explanation of box plots see legend to Figure 3.10, $n=21$ and 19 for reaction and inhibition, respectively). Figure and figure legend adapted from Witkowska and Jahn (2016).

In order to determine whether lipid mixing is a result of a full membrane merger or rather only hemifusion, it is necessary to make sure that the contents of the two fusing vesicles are mixed. This can be done with variety of a content mixing reporters 
(Section 2.5.9). Here syb-SUVs filled with self-quenched concentrations of calcein were used. Upon fusion and content mixing, rapid dilution of the calcein from the SUV lumen into a significantly larger GUV lumen, calcein fluorescence intensity increase will occur. Here, it was assayed for an increase of calcein fluorescence in GUV lumen approximately $30 \mathrm{~min}$ after initiation of a fusion reaction. It could be observed that calcein fluorescence was much higher in the lumen of GUVs that were able to fuse (upper row of images and red box in Figure 3.11), in contrast to $\triangle \mathrm{N}-\mathrm{GUV}$ s that were preincubated with syb 1-96 that suppressed fusion with syb-SUVs (lower row of images and black box in Figure 3.11).

\subsection{SNARE-mediated docking and fusion of single vesicles to $\Delta \mathrm{N}-\mathrm{GUVS}$}

Once the protocol for reconstitution of the $\Delta \mathrm{N}$ complex was set up, the GUV-based assay was used to monitor docking and membrane fusion at the single vesicle level.

\subsubsection{Docking of $\Delta 84$ syb-LUVs on GUV surface}
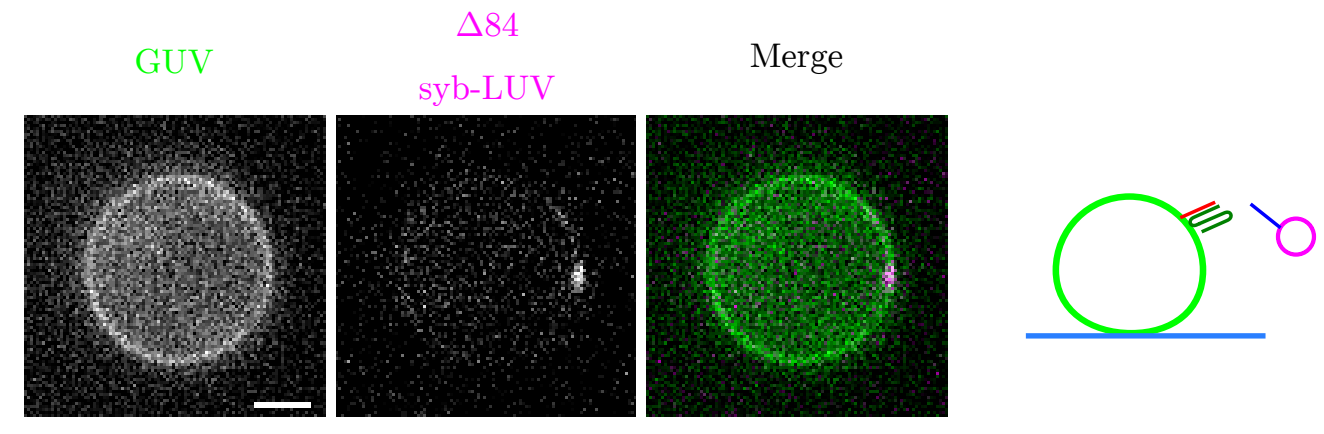

Figure 3.12. Single LUV docked at the $\Delta$ N-GUV membrane. Single LUV (containing $\Delta 84 \mathrm{syb}$ and labelled with TR-PE, magenta) bound to the $\Delta \mathrm{N}-\mathrm{GUV}$ membrane labelled with OG488-PE (green). Scale bar $3 \mu \mathrm{m}$. Figure and figure legend adapted from Witkowska and Jahn (2016).

First aim was to determine how a single syb-LUV looks like docked at the $\Delta$ N-GUV membrane. For this purpose, a syb mutant ( $\Delta 84$ syb; Hernandez et al., 2012) was used that arrests SNARE-mediated fusion at the docking stage (Section 1.2.4). As shown in Figure 3.12, syb-LUV appears as a bright fluorescent spot attached to a GUV membrane. In order to obtain single vesicle resolution, it is necessary to dilute heavily 


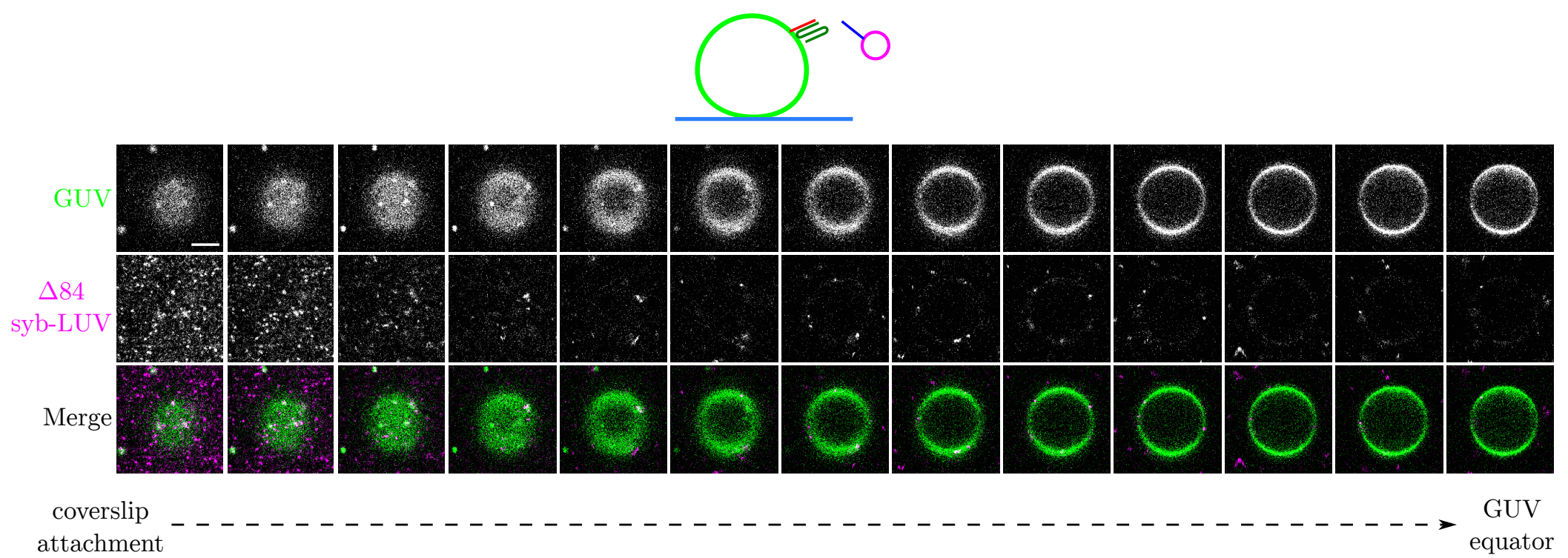

Figure 3.13. Coverslip related artefacts of liposome attachment. A representative $z$-stack showing lower hemisphere of a GUV (labelled with $\mathrm{DiO}$ ) attached to a coverslip after prolonged incubation (30 min) with $\Delta 84$ syb-LUVs (DiD). LUVs dock and diffuse on the GUV membrane, some stay in the solution, and many attach and get immobilised (probably unspecifically) at the coverslip surface. Scale bar $5 \mu \mathrm{m}$, z-slices are separated by $0.6 \mu \mathrm{m}$. Figure and figure legend adapted from Witkowska and Jahn (2016). 
the LUV solution. Additionally, it is hard to capture single vesicles while using long exposure times in a widefield epifluorescent microscope, because such vesicles are diffusing on a GUV membrane (see Section 3.3.2) resulting in a smeared signal of lower fluorescence intensity. It could be also observed, that upon longer incubation $(\sim 30 \mathrm{~min})$ LUVs attach, probably unspecifically, to the coverslip surface (Figure 3.13). Also, if LUVs are mixed with GUVs at the GUV immobilisation stage, they often get trapped at the GUV-coverslip interaction interface or at its rim (Figure 3.13). The coversliprelated effects emphasise the necessity for imaging of vesicle docking and fusion further away from the coverslip surface e.g. at the GUV equatorial plane (see also Section 4.2).

\subsubsection{Single liposome fusion to GUVs}

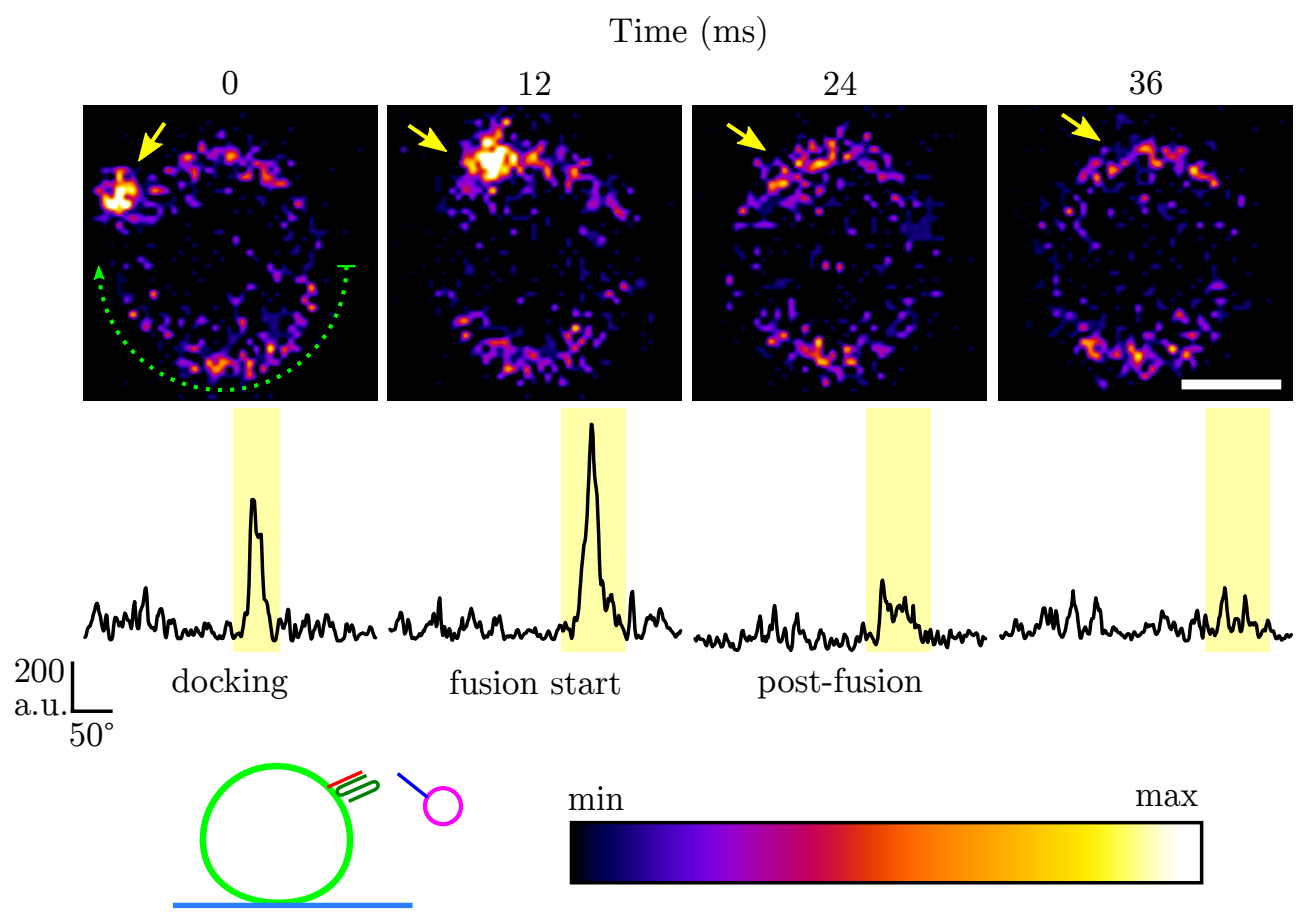

Figure 3.14. Single liposome fusion induced lipid mixing. Image sequence showing an example of a docked LUV (labelled with DiD and indicated with yellow arrow) that fuses with the $\Delta \mathrm{N}-\mathrm{GUV}$ (labelled with $\mathrm{DiO}$, here DiO channel not shown). Scale bar $1 \mu \mathrm{m}$, lookup table for fluorescence intensities shown below the line profiles. The fluorescence intensity profiles below the images were obtained from an approximately $1 \mu \mathrm{m}$-thick segment of the GUV circumference (green dotted arrow indicates line profile start and direction). Fusion is indicated by a transient increase of fluorescence intensity (12 ms), followed by rapid decay, accompanied by spatial broadening of the signal. Figure and figure legend adapted from Witkowska and Jahn (2016). 
For monitoring of single vesicle fusion events by lipid mixing, a WT syb-LUVs labelled with a fluorescent dye DiD were used. WT syb-LUVs readily attached to GUV membrane (time $0 \mathrm{~ms}$ on Figure 3.14) and shortly afterwards fused (12-24 ms on Figure 3.14). Docking and subsequent membrane merger was identified as initial fluorescence intensity peak followed by rapid dilution of the LUV dye (DiD, peak broadening and amplitude drop) in GUV membrane (see line profiles at 12-36 ms presented in lower panel on Figure 3.14).

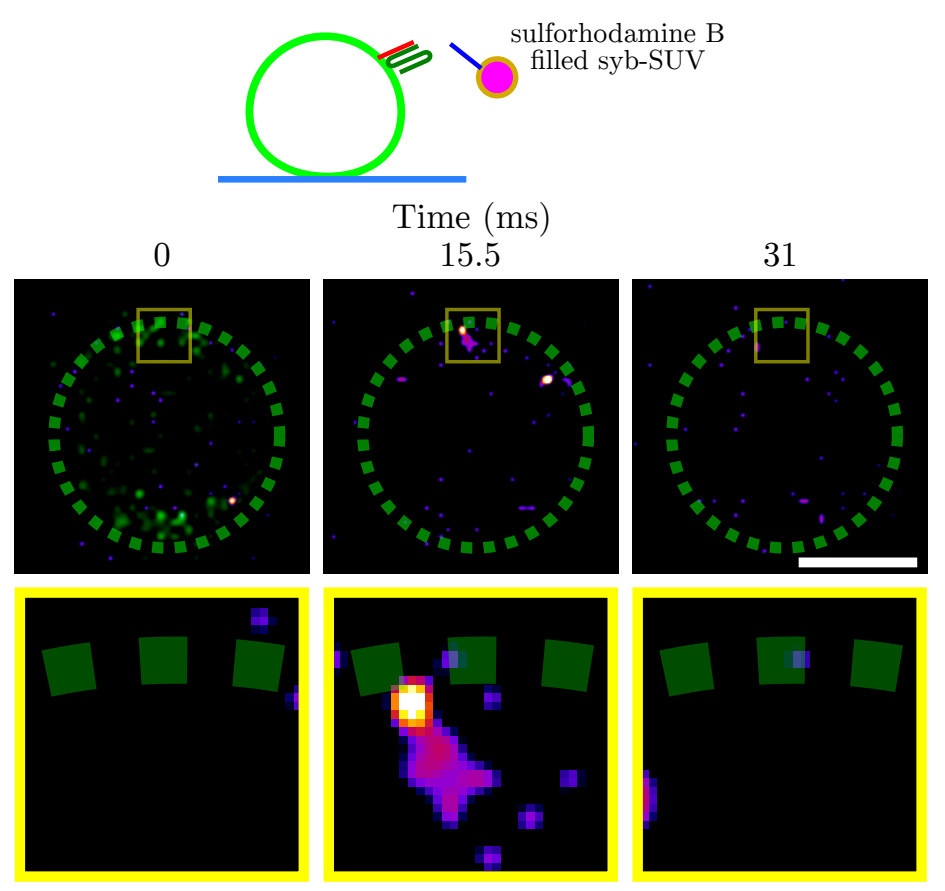

Figure 3.15. Single liposome fusion induced content mixing. Image sequence showing a burst of sulforhodamine B fluorescence directed towards the GUV lumen, which results from fusion of a syb-SUV filled with sulforhodamine B at self-quenching concentration. For clarity, the outline of the GUV (detected in another channel and shown as merged image at time $0 \mathrm{~ms}$ ) is indicated by a green dashed line. Scale bar $1 \mathrm{\mu m}$. Lower panel: Higher magnification of the boxed area in the upper panel. Figure and figure legend adapted from Witkowska and Jahn (2016).

Similar to experiment presented in the Figure 3.11 (Section 3.1.3), the full fusion vs hemifusion was tested with a content mixing indicator. Here, WT syb-SUVs filled with a self-quenched concentrations of sulforhodamine B instead of calcein were used, because of better signal to noise ratio of small fluorescence intensity changes. Likewise calcein on Figure 3.11, sulforhodamine upon content release-mediated dilution increases its fluorescence. Indeed, after addition of syb-SUVs to the imaging chamber with GUVs immobilised, very brief (usually 1 or 2 imaging frames) fluorescence bursts just underneath the GUV membrane could be observed (Figure 3.15). This proves that 
with a GUV-based assay full fusion events between smaller liposomes and GUVs can be monitored.

\subsubsection{Fusion of purified secretory granules to GUVs}



Figure 3.16. Single chromaffin granule fusing to a $\Delta \mathrm{N}-\mathrm{GUV}$. Image sequence showing a chromaffin granule (labelled with TR-PE and indicated with yellow arrow) docking and then fusing with the $\triangle \mathrm{N}-\mathrm{GUVs}$ (visible by a weak labelling in TR channel). Initial TR fluorescence in the GUV membrane is due to fusion events of granules occurring before image acquisition. Scale bar $1 \mu \mathrm{m}$, lookup table as in Figure 3.14. The fluorescence intensity profiles below the images were obtained by a line-scan of the GUV circumference as described in Figure 3.14. Figure and figure legend adapted from Witkowska and Jahn (2016).

Finally, the GUV fusion assay was used to monitor fusion of single purified secretory vesicles. For this purpose, chromaffin granules purified from bovine adrenal glands were used. These vesicles were shown already before to undergo fusion with $\Delta$ N-liposomes (Park et al., 2012b) as they contain endogenous synaptobrevin 2 along with other proteins important for regulated exocytosis such as $\mathrm{Ca}^{2+}$ sensor synaptotagmin-1 (Wegrzyn et al., 2010; Park et al., 2012b). For visualisation, purified granules were labelled with a Texas Red coupled lipid (TR-PE). Such fluorescently labelled vesicles were then 
added instead of liposomes to immobilised GUVs. Even though the imaging buffer (Table A.6) did not contain $\mathrm{Ca}^{2+}$, granules readily attached to the $\Delta \mathrm{N}-\mathrm{GUV}$ membrane also when GUVs were preincubated with a soluble syb fragment (syb 1-96) in order to block acceptor complexes (data not shown). In case of the GUVs that were not preincubated with syb 1-96, granules fused to $\Delta$ N-GUVs with only short delay after docking. Lipid mixing characteristics were similar like for single LUV to GUV fusion (see Figure 3.14) with fluorescence intensity peak on GUV membrane indicating docking and subsequent peak broadening (Figure 3.16).

A

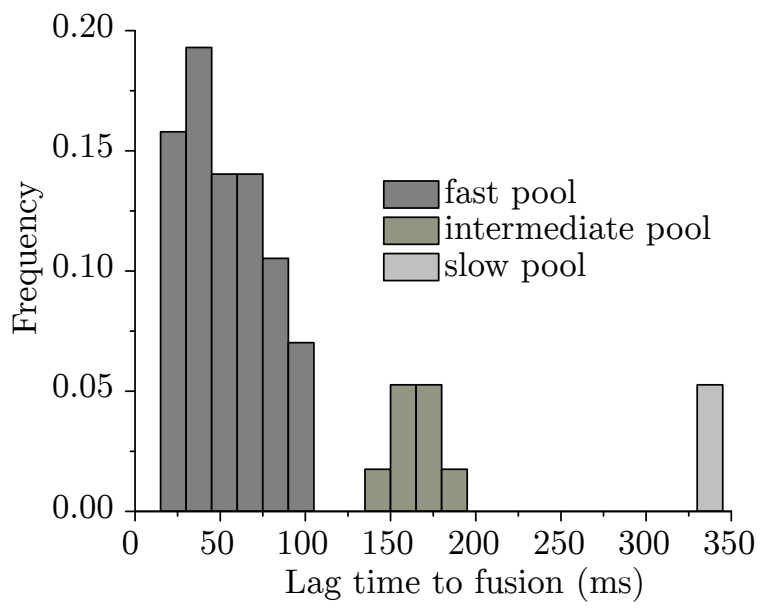

B

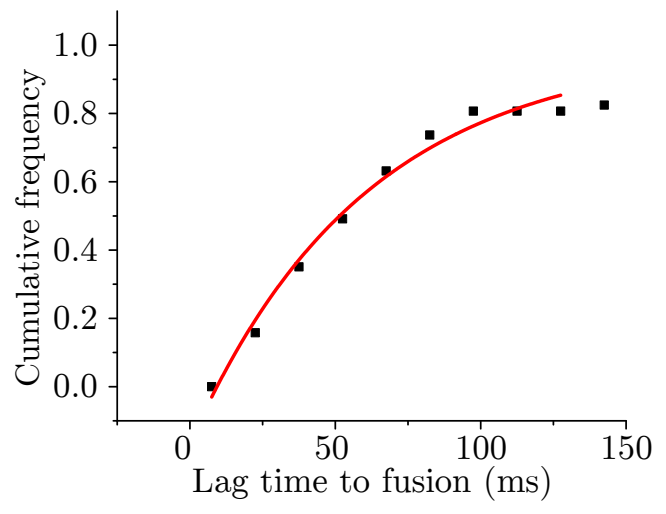

Figure 3.17. Fast fusion kinetics of chromaffin granules. (A) Histogram presenting distribution of lag times between CG docking and fusion (presented total of 57 fusion events, bin size $=15 \mathrm{~ms}$ ). (B) Cumulative distribution of lag times of a fast pool presented in A. The red curve represents a fit with a first order kinetic model (see Section 2.5.8), resulting in a time constant $\tau=60.2 \pm 10.9 \mathrm{~ms}$. Presented 46 fusion events representing fast pool from panel A. Figure and figure legend adapted from Witkowska and Jahn (2016).

Chromaffin granules were fusing very efficiently to GUVs and it was possible to analyse the fusion kinetics of granule population. Closer analysis of lag times between docking and fusion, revealed that at least three kinetic sub-pools can be distinguished (Figure $3.17 \mathrm{~A})$ :

- fast pool - constituting $81 \%$ of the analysed vesicle population, that fuses on average after $51.6 \pm 3.8 \mathrm{~ms}$ (value \pm SEM) after docking;

- intermediate pool - constituting $14 \%$ of the analysed vesicle population, that fuses on average after $161.3 \pm 4.7 \mathrm{~ms}$ after docking; and

- slow pool - constituting $5 \%$ of the analysed vesicle population, that fuses after more than $300 \mathrm{~ms}$ after docking. 
Next, the lag times of the fast pool vesicles were fitted with a first order kinetic model (one component) as in Kiessling et al. (2013; see also Section 2.5.8). The time constant obtained by fitting this model measured $60.2 \pm 10.9 \mathrm{~ms}$ (Figure $3.17 \mathrm{~B}$ ).

\subsection{Investigation of SNARE-mediated vesicle docking}

Observations made in a GUV-based single vesicle assay revealed that syb-LUVs (and especially stalled in a docked state $\Delta 84$ syb-LUVs) after docking diffuse on GUV surface (Figure 3.14 and Section 3.2.1). In order to get some insight into the SNARE-mediated vesicle docking and thus SNARE zippering mechanism, the docking-related phenomena was investigated further with a LUV-GUV system.

\subsubsection{Docking and undocking}

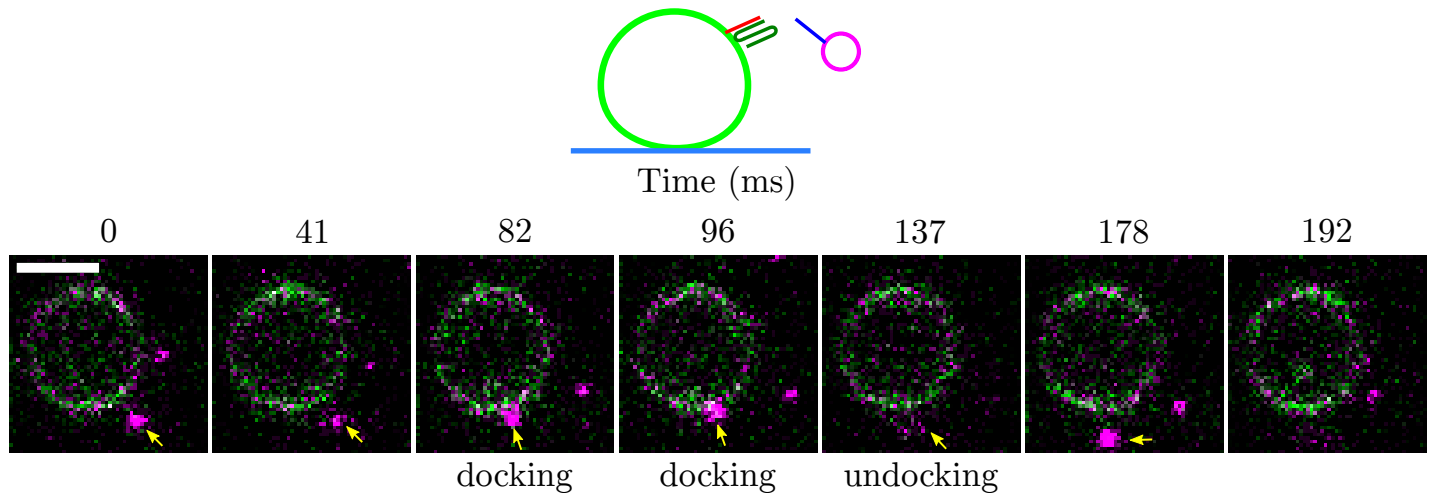

Figure 3.18. Vesicle docking and undocking. Image sequence presenting a representative example of transient interactions of a CG (labelled with TR-PE, shown in magenta and marked with yellow arrow) with $\triangle \mathrm{N}$-GUV membrane (labelled with DiO, shown in green). Scale bar $2 \mu \mathrm{m}$.

One observation made during search for the single vesicle fusion events was reversible interaction of WT syb-LUVs with $\Delta \mathrm{N}-\mathrm{GUV}$ membrane (as shown in Figure 3.18). Such interactions may occur independently of SNARE proteins due, to random collisions of LUV with GUV membrane, or due to interactions of SNARE proteins with the lipids of another vesicle. In order to check the character of this process, time from docking of a WT syb-LUV on a GUV to undocking (labelled on a graph as docking time) was compared for 3 groups of GUVs: $\Delta \mathrm{N}-\mathrm{GUVs}, \Delta \mathrm{N}-\mathrm{GUVs}$ that were preincubated with syb 1-96 (and thus contained ternary SNARE complex not eligible for syb binding any more - inhibited $\Delta \mathrm{N}-\mathrm{GUVs}$ ), or GUVs that did not contain any protein (protein 


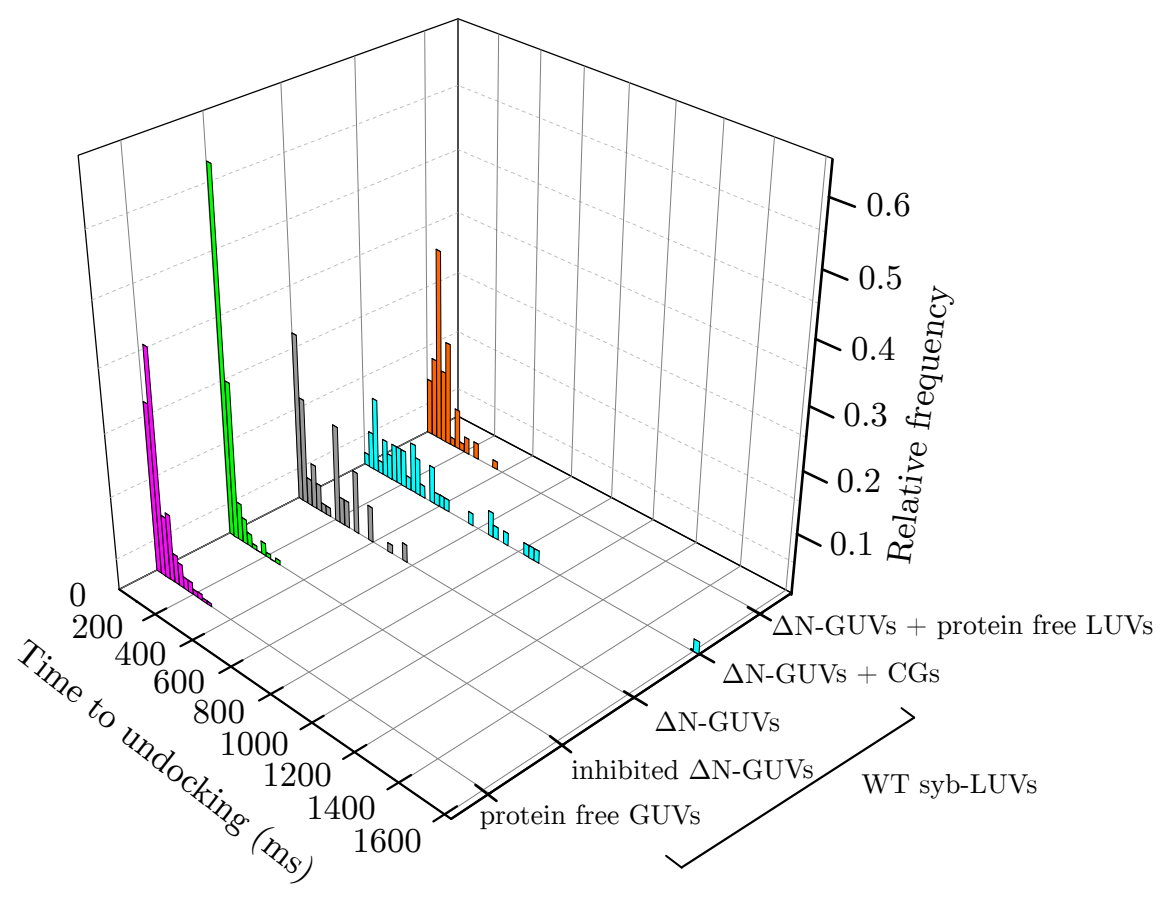

Figure 3.19. Reversible interactions of LUVs and GUVs. Histograms presenting times of interaction of liposomes and CGs, with the membrane of GUVs. Number of analysed syb-LUVs: protein free GUVs -209 , inhibited $\Delta \mathrm{N}-\mathrm{GUVs}-145$, $\Delta \mathrm{N}-\mathrm{GUVs}-69$; number of analysed CGs - 49; number of protein free LUVs - 75 . Bin size $=25 \mathrm{~ms}$.

free GUVs); as well as for: WT syb-LUVs, CGs, and protein free LUVs. The result of this experiment (Figure 3.19) indicates that presence of a $\Delta \mathrm{N}$ complex on GUV membrane can lead to prolonged interaction times (>250 ms) with vesicles containing syb before undocking, and not to fusion, while interaction times shorter than $\sim 250 \mathrm{~ms}$ are probably coming from other interactions (i.e. random collisions or SNARE-lipid interactions).

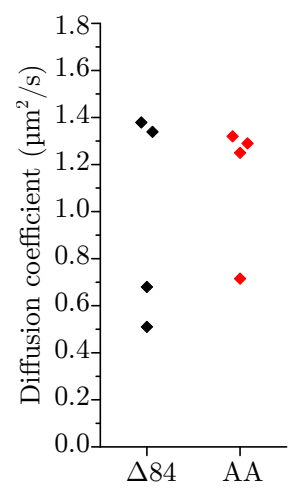

Figure 3.20. Docked vesicle mobility on the GUV surface. Diffusion coefficients of the docked vesicles obtained from MSD calculations for $\Delta 84$ syb-LUVs and AA syb-LUVs. Dots represent single tracked LUVs. 


\subsubsection{Characterisation of mobility of docked vesicles}

A brightfield
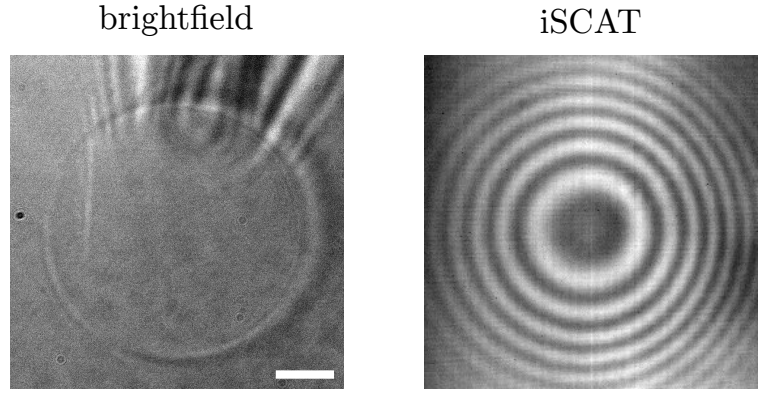

B

positive peak amplitude

negative peak amplitude
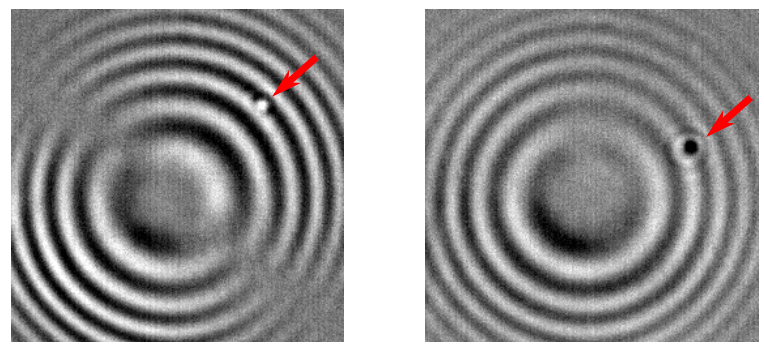

Figure 3.21. Imaging of $\Delta \mathrm{N}-\mathrm{GUVs}$ with iSCAT microscopy. (A) On the left: A brightfield image of a $\Delta \mathrm{N}-\mathrm{GUV}$ aspirated with a micropipette (dark shadow in the upper part of the image) for immobilisation. Scale bar $10 \mu \mathrm{m}$. On the right: An iSCAT image of the same GUV. Rings represent the iSCAT signal (interference pattern) of the GUV sphere. (B) Example iSCAT images showing $\triangle \mathrm{N}-\mathrm{GUV}$ with a docked syb-LUV (marked with a red arrow) visible as a spot whose intensity could be fitted with either positive (left, white spot) or negative (right, black spot) peak amplitude depending on its position relative to the light interference pattern. The iSCAT imaging and analysis was performed together with Susann Spindler (group of Prof. Vahid Sandoghdar, Max Planck Institute for the Science of Light, Erlangen, Germany).

As already mentioned before in Section 3.2.1, LUVs that were arrested in a docked state were diffusing on the membrane of $\Delta \mathrm{N}-\mathrm{GUVs}$. Among these there were $\Delta 84 \mathrm{syb}-\mathrm{LUV}$, AA syb-LUVs (see below and Section 1.2.4), as well as WT syb-LUVs in the presence of low $\Delta \mathrm{N}$ complex concentration available for SNARE complex formation. Next, mobility of syb-LUVs docked on $\Delta \mathrm{N}-\mathrm{GUVs}$ was investigated (Section 2.5.10). Here, the relatively small size of GUVs prepared with an optimised protocol (see Section 3.1.1) was advantageous, since it spatially limited diffusion surface of a vesicle and allowed faster imaging due to smaller size of an $x y$ image (less pixels), as well as less $z$-stacks that needed to be acquired to capture the whole GUV. Measurement of mobility of docked $\Delta 84$ syb-LUVs and AA syb-LUVs (a syb zippering mutant I45A M46A that was previously shown to have impaired $\Delta \mathrm{N}$ complex binding; Wiederhold et al., 2010; see also Section 1.2.4) showed that their diffusion coefficients are in a range from 0.5 
to $1.4 \mathrm{\mu m}^{2} / \mathrm{s}$ (Figure 3.20). No differences between both syb mutants could be observed, but it has to be acknowledged that sample number was very small (4 liposomes in each condition) due to very time-consuming analysis including manual track curation in thousands of frames.

A

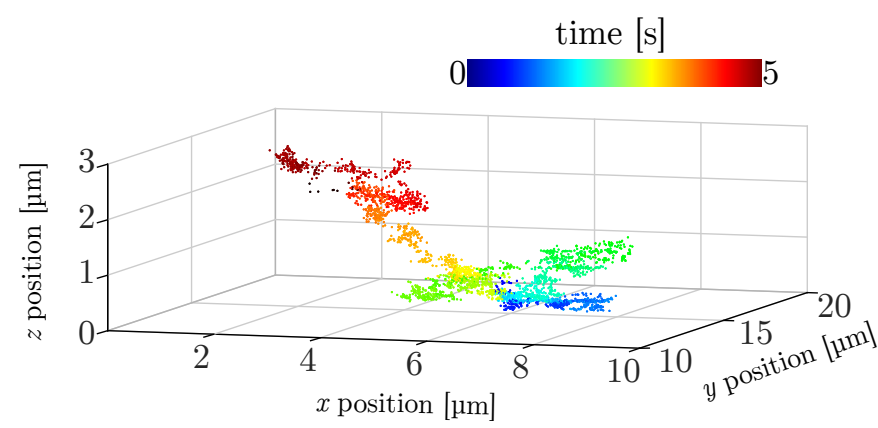

B

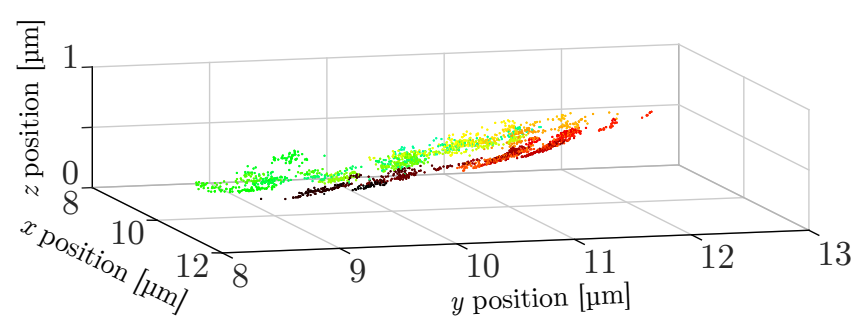

C

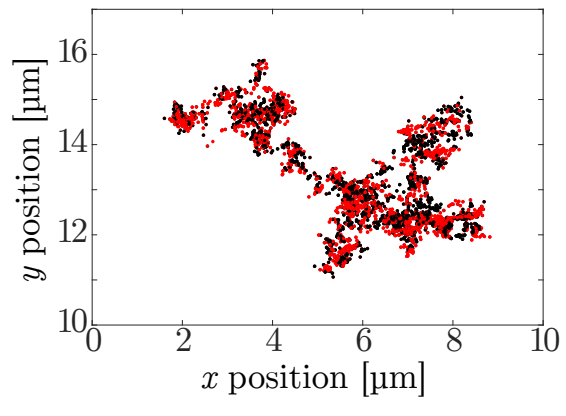

D

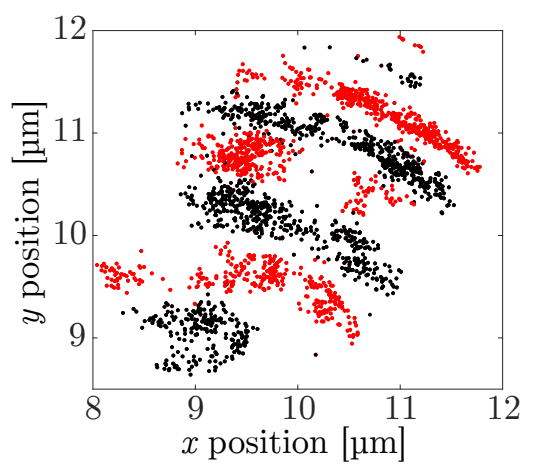

Figure 3.22. Tracking of LUVs docked on the on the GUV surface with the use of iSCAT microscopy. (A-B) Representative scatter plots presenting $x y z$ trajectories of single syb-LUVs docked on the GUV surface. Point colour represents localisation time during a $5 \mathrm{~s}$ track (temporal colour code bar on top of panel $\mathrm{A}$ ). $(\mathrm{C}-\mathrm{D})$ The $x y$ projections of scatter plots from panels A-B (time omitted). Point colour depends on the modulation of the LUV iSCAT signal intensity reflecting fluctuations in the LUV $z$-position relative to the GUV membrane. Black points are fitted with a negative, and red with a positive peak amplitude (as explained in Figure $3.21 \mathrm{~B}$ ). Classification of the docking type for a loosely (C) and tightly (D) docked liposomes was done based on these projection graphs (see description in text). The iSCAT imaging and analysis was performed together with Susann Spindler (group of Prof. Vahid Sandoghdar, Max Planck Institute for the Science of Light, Erlangen, Germany).

Since the time resolution for 3D localisation of docked vesicle was not so high, there was only one LUV localisation during a $z$-stack allowed, and also due to a very timeconsuming data analysis, we turned to the interferometric scattering (iSCAT) microscopy (see Section 2.5.2) that allows very fast, label-free, 3D imaging, with very accurate particle localisation. This set of experiments was performed together with 
Susann Spindler (group of Prof. Vahid Sandoghdar, Max Planck Institute for the Science of Light, Erlangen, Germany). The iSCAT visualisation allowed imaging of single GUVs with rings representing spherical GUV membrane, resulting from interference between scattered field and reference beam (Figure 3.21 A, for iSCAT signal generation principle see Figure 2.5). Next, we attempted visualisation of single docked vesicles. Docked LUVs appeared as small circular spots overlapping with the GUV membrane, with an iSCAT signal intensity peak that was either positive or negative, depending on the liposome $z$-localisation (see panel B in Figure 3.21). These docked LUVs were tracked, as they were diffusing attached to the GUV membrane, in three dimensions and with high spatiotemporal resolution (for example trajectories see Figure 3.22). Obtained trajectories were then used for determination of: liposome diffusion coefficients (Figure 3.24, for method description see Section 2.5.10); and local mobility of the docked LUVs in the $z$-direction, based on which classification of the docking type was made (Figure 3.22 C-D and 3.23).

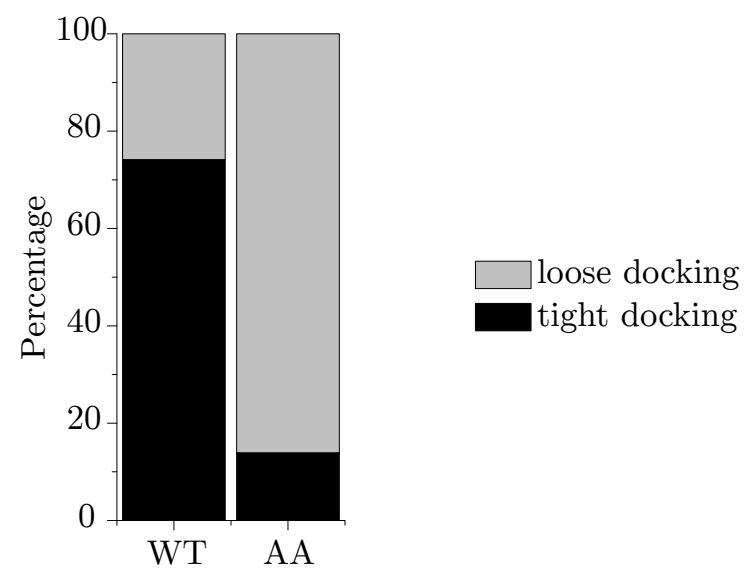

Figure 3.23. Docking mode occurrence depends on the SNARE zippering degree. Comparison of percentages of LUV trajectories obtained with iSCAT microscopy, classified as either loosely (grey) or tightly (black) docked, with regards to the reconstituted synaptobrevin variant (either WT or AA syb). Number of trajectories analysed: $n_{\mathrm{WT}}=147$ and $n_{\mathrm{AA}}=115$. The iSCAT imaging and analysis was performed together with Susann Spindler (group of Prof. Vahid Sandoghdar, Max Planck Institute for the Science of Light, Erlangen, Germany).

Local mobility of the docked LUVs in the $z$-direction can be determined by analysing their iSCAT signal modulation (positive or negative peak amplitude, as can be seen in Figure $3.21 \mathrm{~B}$ ). Already first analyses of tracks of syb-LUVs docked on GUVs revealed that there is no single docking mode (Figure $3.22 \mathrm{C}-\mathrm{D}$ ). It seems that some LUVs, despite appearing as being docked on the GUV membrane, exhibit fluctuations in $z$-position with regards to the GUV surface as can be determined by the often changing 


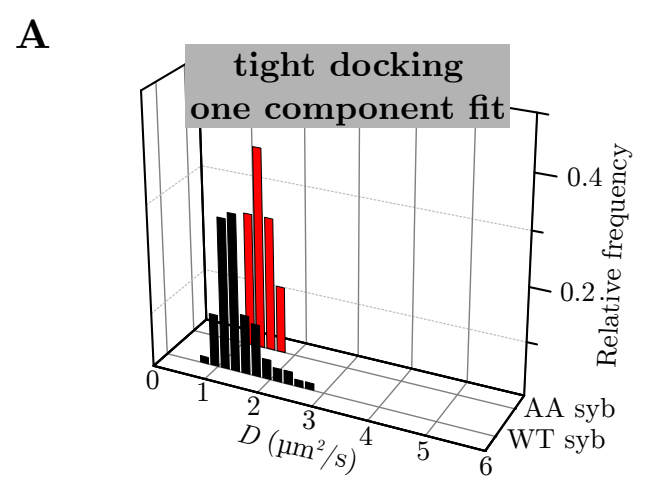

B

D
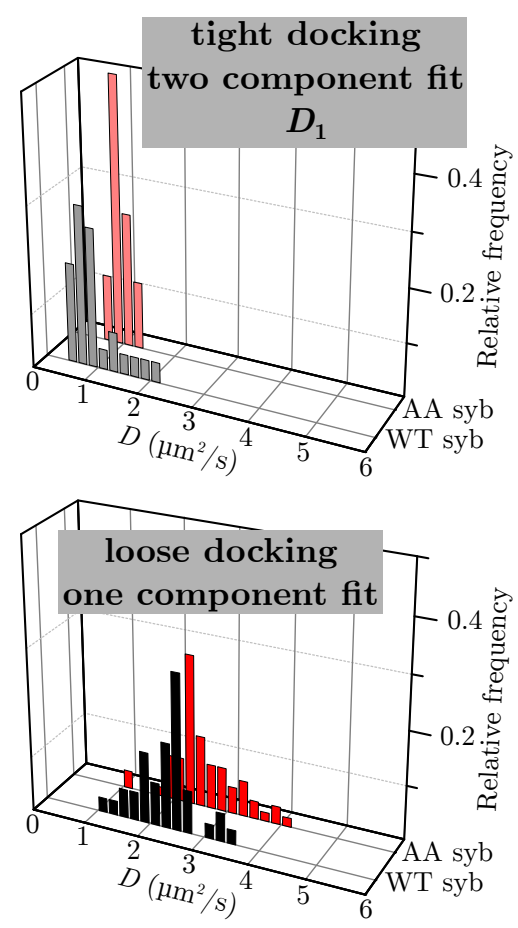

C
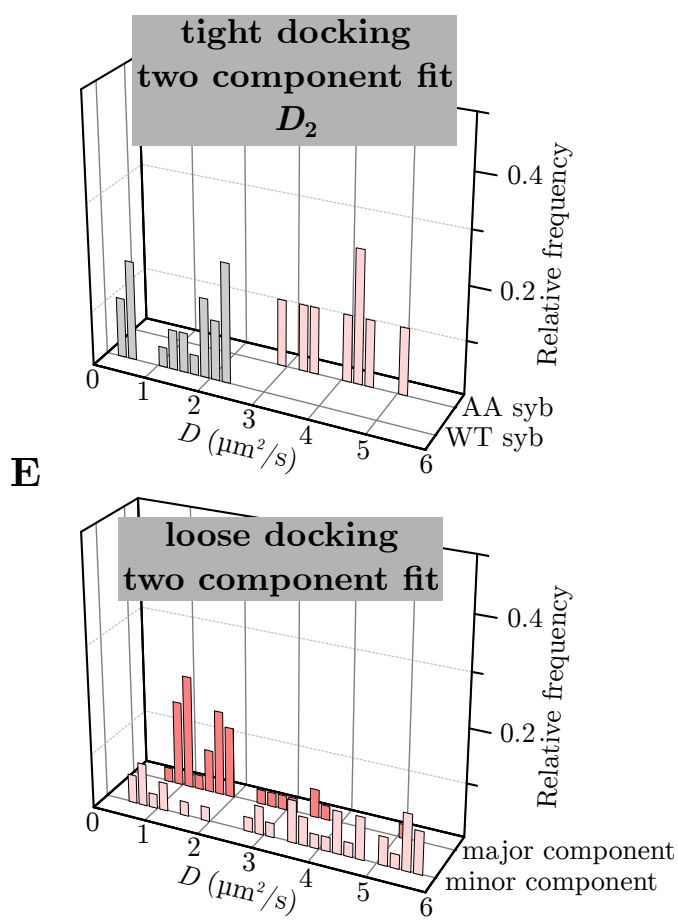

Figure 3.24. Comparison of diffusion coefficients of syb-LUVs docked on $\Delta \mathrm{N}-$ GUVs investigated by iSCAT. All histograms present relative frequency of diffusion coefficients values calculated for either WT syb-LUVs (grey palette) or AA syb-LUVs (red palette) docked on $\triangle \mathrm{N}$-GUVs. Diffusion coefficients were determined according to Equation 14. (A-C) Tightly docked vesicles with diffusion coefficients fitted either with a 1 component equation (A) or with 2 component equation (B-C) showing either major (B) or minor (C) component. (D-E) Diffusion coefficients of loosely docked vesicles fitted with 1 (D) or 2 (E) component equation. Loosely docked WT syb-LUVs were only fitted with 1 component and therefore graph in panel E presents major (darker bars, $D_{1}$ ) and minor (lighter bars, $D_{2}$ ) components for AA syb-LUVs. The number of analysed trajectories: (A) $n_{\mathrm{WT}}=82$ and $n_{\mathrm{AA}}=8$; $(\mathrm{B}-\mathrm{C}) n_{\mathrm{WT}}=27$ and $n_{\mathrm{AA}}=8 ;(\mathrm{D}) n_{\mathrm{WT}}=38$ and $n_{\mathrm{AA}}=61$; and (E) $n_{\mathrm{AA}}=38$. The iSCAT imaging and analysis was performed together with Susann Spindler (group of Prof. Vahid Sandoghdar, Max Planck Institute for the Science of Light, Erlangen, Germany). 
peak amplitude sign of the fitted iSCAT signal (Figure 3.22 C, loose docking). On the other hand, there is also a group of liposome trajectories that align tightly with the GUV surface and the peak amplitude sign changes align with the GUV pattern (tight docking, see panel D in Figure 3.22). In order to test how such docking behaviour relates to SNARE complex zippering, trajectories of docked LUVs with reconstituted synaptobrevin variants exhibiting normal or impaired zippering (either WT syb or AA syb) were compared. All recorded tracks were then unambiguously classified as either loosely or tightly docked. Quantification of the number of vesicles (trajectories) displaying either loose or tight docking, revealed that $74 \%$ of WT syb-LUVs were tightly docked, while $86 \%$ of AA syb-LUV were docked loosely (Figure 3.23).

Next, the diffusional mobilities of differentially docked vesicles were analysed in more detail. From trajectories, a cumulative probability of squared displacement $\left(P\left(r^{2}\right)\right)$ was calculated, and fitted to either one or two component diffusion equation (see Section 2.5.10; and Hsieh et al., 2014). Diffusion coefficients calculated for tightly docked vesicles (Figure $3.24 \mathrm{~A}-\mathrm{C}$ ) had an average value of $1.35 \mathrm{\mu m}^{2} / \mathrm{s}$ when fitted with a one component equation (Equation 14, $\varepsilon_{2}=0$ ). There, no significant difference between AA syb and WT syb-LUVs could be detected (Figure 3.24 A). However, when 2 component fit had to be utilised (Equation $14, \varepsilon_{2} \neq 0$ ), $D_{2}$ calculated for AA syb-LUVs had much higher values i.e. $4 \pm 0.26 \mathrm{\mu m}^{2} / \mathrm{s}$ in comparison to $D_{2}$ of WT syb-LUVs being in between $0.34-2.33 \mathrm{\mu m}^{2} / \mathrm{s}$ (Figure $3.24 \mathrm{~B}-\mathrm{C}$ ). On the other hand, loosely docked vesicles diffused faster than the tightly docked ones, with an average diffusion coefficient of 2.3-2.4 $\mathrm{m}^{2} / \mathrm{s}$ (Figure 3.24 D). Only loose AA syb-LUVs (but not loose WT syb-LUVs) were fitted with a 2 component equation (as mentioned in Section 2.5.10, distinction between one or two component fitting was done based on the fit quality), and here, major and minor components seemed to be more equally spread (between $0.52-5.74 \mathrm{\mu m}^{2} / \mathrm{s}$, Figure $3.24 \mathrm{E}$ ) in comparison to the tight mode. 



\section{Discussion}

\subsection{Effect of the preparation method on the GUV quality}

Giant unilamellar vesicles were first described by (Reeves and Dowben, 1969) but only in the last 15 years they gained more attention as a model system for both membrane mechanics, as well as biological membranes (Méléard et al., 2009). The main advantages of a GUVs as model system are: (i) the ability of visualisation of such vesicles with basic microscopy techniques, (ii) the GUV membrane (even if GUVs are immobilised) is largely free of curvature stress, (iii) the surface interaction artefacts can be neglected (Section 4.2), and (iv) GUVs are suitable for micromanipulation and have easy to control membrane tension. One of the reasons for the delay of GUVs becoming a model system for biological membranes was a fact that the preparation methods are not universal for wide range of lipid compositions, buffers, and proteins. That is why, protocols for GUV formation likely have to be established for each type of preparation separately. Nevertheless, a constantly growing number of publications present use of GUVs as biological membrane mimics in in vitro reconstitution systems. These include the initial studies with reconstitution of bacteriorhodopsin (Manneville et al., 1999), investigation of ion channels (see e.g. Aimon et al., 2011), ESCRT machinery (see e.g. Wollert et al., 2009), autophagy (see e.g. Kaufmann et al., 2014), and many more. In this study, a GUV-based single vesicle fusion assay was developed.

A range of GUV formation conditions (Table 3.1) was tested for formation of GUVs with reconstituted $\Delta \mathrm{N}$ complex in order to develop a reproducible protocol for efficient formation of $\Delta \mathrm{N}-\mathrm{GUVs}$ of appropriate size, lamellarity, protein reconstitution efficiency, and yield. Many of the conditions tested (like formation of GUVs in the presence of detergents) did not yield GUV formation at all, others (like GUV formation in buffers of physiological salinity) yielded GUVs but the reconstituted protein lost its activity. Finally, some procedures that were supposed to improve the GUV preparation (like sucrose addition at the dehydration step for improving protein activity), did not noticeably improve the GUV quality. The GUVs obtained from the final protocol (Table 3.1), utilised throughout this study, were thoroughly characterised with regards to their morphology (size, lamellarity), as well as protein content and activity. $\Delta \mathrm{N}-\mathrm{GUVs}$ were $2-8 \mu \mathrm{m}$ in diameter (a bit larger when platinum electrodes were utilised instead of ITO slides like in experiments with iSCAT microscopy in Section 3.3.2) and mostly unilamellar (Figure 3.4). The reconstitution efficiency of the $\Delta \mathrm{N}$ complex 
was very high $(\sim 100 \%$, Figure 3.5$)$ and the protein was shown to be active and fusioncompetent (Section 3.1.3). What is more, the diffusion coefficients of proteins and lipids (around $1.2 \mathrm{\mu m}^{2} / \mathrm{s}$ and $2.8 \mathrm{\mu m}^{2} / \mathrm{s}$ respectively) in this GUV preparation is comparable with values obtained in other studies utilising SNARE-GUVs (although with different acceptor SNARE complex construct and slightly different lipid composition) such as (i) determined by FCCS diffusion coefficients in Bacia et al. (2004), and (ii) determined by FRAP diffusion studies by Motta et al. (2015) and in Pincet et al. (2016). The protocol developed in this project was also reproduced in another laboratory (by Susann Spindler in the group of Prof. Vahid Sandoghdar, Max Planck Institute for the Science of Light, Erlangen, Germany) from $\Delta \mathrm{N}-\mathrm{SUVs}$ prepared in our group but with equipment and buffers prepared externally, using both, ITO slides (Angelova et al., 1992) and platinum electrodes (Angelova and Dimitrov, 1986).

One has to take into account that some of the parameters were not fully tested due to time constraints of the project, and due to this fact $\triangle \mathrm{N}$-GUV preparation procedure could be still improved. One example is the detergent-mediated reconstitution of the $\Delta \mathrm{N}$ complex into preformed GUVs. A similar method is widely utilised for reconstitution of proteins into LUVs (for comparison see Section 2.3.2). Although, the exact mechanism of proteoliposome formation is still not fully understood (Rigaud and Lévy, 2003), the method should in principle also work for GUVs. This has been already demonstrated by Dezi et al. (2013) with DOTM-mediated reconstitution of functional proteins. However, as known already from LUV reconstitution protocols, not all detergents and detergent concentrations fit all proteins (Rigaud and Lévy, 2003; Hernandez et al., 2012), and that is why, a wider range of detergents as well as their concentrations with regards to lipid content in the samples (so called $R$-value, Rigaud and Lévy, 2003; Hernandez et al., 2012) have to be tested. In this study, 9 detergents were tested but each at only one concentration. Testing a wider concentration range, might have led to successful GUV formation with reconstituted $\Delta \mathrm{N}$ complex.

In the course of GUV formation protocol optimisation, there were also factors tested that were shown before to improve preservation of protein activity upon dehydration step. The procedure of adding disaccharides or ethylene glycol was shown before to protect proteins from the dehydration-related activity loss and maintenance of membrane structure (Crowe et al., 1988; Keller et al., 1988; Doeven et al., 2005). In this method sugar is believed to replace water in the drying sample and bind to lipid headgroups, as well as to form hydrogen bonds with the protein, and in effect to prevent aggregation and exclusion of integral membrane proteins from the membrane (Crowe et al., 1988). In experiments presented in this work, there was no noticeable improvement 
in the GUV quality upon sucrose addition, with regards to GUV morphology, as well as protein activity. However, SNARE proteins (especially the constructs used in this study) are known to be stable in many different conditions due to their unstructured nature in the native single protein state, and fold only upon SNARE-complex formation (Fasshauer et al., 1997a,b; Margittai et al., 2001; Hazzard et al., 1999). Therefore, it may not be excluded that the $\Delta \mathrm{N}$ complex preserves equally well in all conditions but disaccharide addition at the membrane dehydration step would be beneficial for other proteins.

Since proteo-GUVs are more and more popular as a biological membrane model, and due to the fact that GUV formation protocol very often has to be adjusted, there is an urgent need for development of more standardised protocol optimisation procedures. It is usually very hard to compare various protocols simply due to the fact that reporting of the parameters is not full or not consistent. For example, protocols often do not contain details about chamber design - in case of ITO slides, there is no information about the slide resistance and/or the spacer thickness (that is necessary for electric field calculation), and in case of platinum electrodes about the axial distance and the wire thickness. The utilised voltage settings depend hardly on the chamber geometry and it is often not stated whether reported values are $V_{\mathrm{pp}}$ (peak-to-peak) or rather $V_{\mathrm{RMS}}$ (root mean square), or even whether the used function is sinusoidal at all. What is more, many publications fail to report the GUV quality factors like: size, lamellarity, protein content and activity. In this study, efforts were made to report all important protocol parameters and to test the GUV quality.

An interesting alternative for GUV formation is a method presented by Richmond et al. (2011). A special design of an acrylic chamber allows formation of an asymmetric planar bilayer with reconstituted proteins at desired orientation. This is then followed by microfluidic jetting that forms a GUV maintaining membrane asymmetry and with desired luminal buffer. However, such setup requires specialised equipment, and does not yield many GUVs (formation of one GUV at a time). Furthermore, in the study describing this method (Richmond et al., 2011), lipid and protein mobilities in GUVs were not reported, so it is hard to asses their quality. 


\subsection{A novel platform for monitoring single vesicle fusion with high temporal and spatial resolution}

In order to monitor single vesicle fusion in vitro, generally two approaches were developed (see also Section 1.3.2). The first one involves surface immobilisation of small vesicles (Yoon et al., 2006; Diao et al., 2010; Kyoung et al., 2011), and the second one uses planar membranes deposited on functionalised surfaces (Domanska et al., 2009; Karatekin et al., 2010; Schwenen et al., 2015). However widely used, these assays have some disadvantages (Section 1.3.2). One of the drawbacks is that contact with the surface can restrict free lateral diffusion of membrane components, i.e. proteins and lipids. Another one, is the effect of curvature or membrane tension on membrane fusion energy landscape. Various strategies were used to minimise artefacts, such as surface functionalisation minimising friction (Karatekin et al., 2010) or use of porous substrates yielding adhesion-free membrane suspended across the pores (Schwenen et al., 2015). The assay presented in this work employs GUVs mimicking the plasma membrane instead of highly curved vesicles (e.g. Kyoung et al., 2011) or planar membrane bilayers (e.g. Domanska et al., 2009; Schwenen et al., 2015) that allows monitoring of single fusion events on a free-standing membrane with a high spatiotemporal resolution.

The first step to develop a GUV fusion assay was successful formation of GUVs containing a stabilised SNARE acceptor complex ( $\Delta \mathrm{N}$ complex, Figure 3.1). The $\Delta \mathrm{N}$ complex reconstituted into GUVs was then shown to be able to form functional ternary SNARE complexes and induce membrane fusion (Figure 3.7-3.9). Further, the assay was optimised for single vesicle visualisation (Figure 3.12). Notably, many vesicles attached to the coverslip surface, probably due to unspecific interactions (Figure 3.13), and despite the hydrophilic nature of coverslip functionalisation. Such unspecific attachment can become a major drawback in assays with immobilised small vesicles where signal detection relays on small fluorescence intensity changes at the coverslip surface (like e.g. Kyoung et al., 2011). In the method presented here, the unspecific vesicle adhesion can be neglected due to the fact that fusion measurements are done at the equatorial plane of the GUV, and therefore micrometers away from the coverslip. Observation at the equatorial plane also assures that the membrane taking part in docking and fusion is largely tension- (Gleisner et al., 2016) and curvature-free. What is more, this membrane geometry also lacks diffusional constrains and local membrane distortions, like in assays with small vesicles (e.g. Kyoung et al., 2011).

The synaptobrevin containing liposomes, as well as purified secretory vesicles readily attached to $\triangle \mathrm{N}-\mathrm{GUVs}$ and fused in milliseconds after docking (Figure 3.14 and 3.16). 
So far, only one in vitro fusion assay by Domanska et al. (2009) has reported fusion events with comparable kinetics (with SUV to supported membrane bilayer fusion after $8 \mathrm{~ms}$ ). In the assay presented in this study, LUVs fused to GUVs even in one frame after docking (i.e. around $12 \mathrm{~ms}$ ). It was shown before that SUVs and LUVs undergo fusion with different kinetics due to the different curvature (Hernandez et al., 2012; higher curvature lowers the energy needed for fusion, Kozlov et al., 2010), and SNARE complexes cooperativity (Hernandez et al., 2014). That means, that GUV-based assay has potential to observe fusion events either equally fast or even faster then in the assay by Domanska et al. (2009), especially taking into account that observations were performed at the time resolution limit of the used equipment and existence of faster events cannot be excluded. The small vesicles (i.e. around $40 \mathrm{~nm}$ in diameter; Schuette et al., 2004) were not used in this study (except for content release assays Figure 3.11 and 3.15), because they were harder to observe with confocal microscopy due to the smaller size. The signal of liposome dye dilution in the GUV membrane was small even for larger vesicles (around $100 \mathrm{~nm}$ in diameter; Hernandez et al., 2012) also because of non-parallel GUV membrane orientation with regards to the focus plane. On the other hand, small vesicles were used for the content release experiments because they are less leaky when a content dye is incorporated (personal communication with Dr. Pradip Kumar Tarafdar, Göttingen, now Kolkata, India). It needs to be mentioned that the three dimensional geometry of GUVs makes data acquisition and analysis more challenging in comparison to assays with fusion surface corresponding to the focus plane. Here, usually 3-4 z-planes were acquired in order to capture vesicles that after docking were rapidly diffusing on the GUV surface prior to fusion (like can be seen in Figure 3.14, post-docking diffusion is further discussed in Section 4.3).

Notably, the delay time between docking and fusion of chromaffin granules was at or below the assay resolution limit (around $12 \mathrm{~ms}$, see Figure 3.17). This becomes intriguing when one takes into account that neither $\mathrm{Ca}^{2+}$ nor PIP2 were present in the reaction. These factors were shown before to enhance fusion speed in an in vitro bulk fusion assay (Park et al., 2012b). However, in such an assay docking and fusion steps cannot be discriminated from each other (Section 1.3.2), and therefore, if synaptotagmin-mediated effect is at the docking step this would not result in decreased docking-to-fusion lag times in a GUV-based assay, but rather higher docking efficiency. On the other hand, the few milliseconds delay is in a similar range as observed between calcium triggering and fusion measured with capacitance patch clamping (Voets, 2000). In this kind of assay however, vesicles could be potentially docked at the plasma membrane prior to the trigger signal. There were also studies with fluorescently la- 
belled chromaffin granules and TIRF microscopy reporting fusion of previously not docked vesicles (so called "crash" fusion) with time of $33 \mathrm{~ms}$ (Verhage and Sørensen, 2008). Moreover, comparable delays were also previously observed in assay involving planar bilayers and either SUVs (minimum $8 \mathrm{~ms}$; Domanska et al., 2009) or purified synaptic vesicles (time constant of 36 and $63 \mathrm{~ms}$ for condition with and without calcium, respectively; Kiessling et al., 2013). The chromaffin granule fusion time constant measured in this study $(\tau \approx 60 \mathrm{~ms})$ is close to synaptic vesicle to planar membrane fusion without calcium. Therefore, taking into account (similarly like with SUV and LUV fusion) the substantial vesicle diameter difference (around $40-50 \mathrm{~nm}$ for synaptic vesicles, Takamori et al., 2006; and 100-300 nm for chromaffin granules, Park et al., $2012 \mathrm{~b}$ ) and the effect of curvature and cooperativity (as discussed earlier in this section), GUV-based assay may have potential of promoting faster fusion events then with planar bilayers.

However, few milliseconds delay presented in this work, although is one of the shortest delay times among in vitro fusion assays, is still slower than sub-millisecond triggered synaptic vesicle fusion events (Neher and Sakaba, 2008). There is already a substantial amount of electrophysiological evidence that such events are not only coming from previously docked vesicles but also from "newcomers" (e.g. Hallermann et al., 2010; Ritzau-Jost et al., 2014) that need to undergo rapid docking and priming prior to fusion. Nevertheless, such fast events so far could not be spotted in cells with microscopy techniques like in studies by Zenisek et al. (2002) or Midorikawa and Sakaba (2015), probably due to technical constraints. There can be couple of reasons why the fusion speed in the GUV-based assay is slower than in vivo:

1. Reaction temperature - All microscopy measurements presented in this study were performed at the room temperature. It was demonstrated before that syb 49-96 displacement kinetics from the $\Delta \mathrm{N}$ complex during SNARE ternary complex formation is temperature sensitive and therefore temperature has an effect on fusion times (Hernandez, 2012). Moreover, the temperature dependence of neurotransmission has been known since a long time (Eccles et al., 1941) and was investigated in many contexts including fast mammalian synapses (Sabatini and Regehr, 1996) and exocytosis in PC12 cells (Zhang and Jackson, 2008). Accordingly, increasing the reaction temperature to the physiological level $\left(36-37^{\circ} \mathrm{C}\right)$ in a GUV assay could potentially increase fusion speed, and therefore, decrease observed lag times. 
2. Artificial acceptor complex - The stabilised acceptor complex (Pobbati et al., 2006) used in this study consists of syntaxin, SNAP-25 and a short fragment of synaptobrevin (49-96). In cells such complex does not exist, but at the same time the arrangement of plasma membrane SNARE-proteins (i.e. syntaxin and SNAP-25) along with accessory proteins prior to arrival of the synaptic vesicle is not fully understood and still under debate. The $\Delta \mathrm{N}$ complex used here is believed to increase the SNARE complex zippering nucleation efficiency and therefore vesicle docking rate (Pobbati et al., 2006; Smith and Weisshaar, 2011; but the docking efficiency was not determined in a GUV assay) and assure the correct SNARE complex assembly (parallel versus antiparallel; Choi et al., 2016). However, the necessity of syb 49-96 displacement prior to full zippering creates an additional energetic barrier increasing time needed after initiation for full SNARE complex assembly (also due to partial reversibility of the N-terminal zippering; Fasshauer et al., 2002; Wiederhold and Fasshauer, 2009; Hernandez, 2012 also discussed in Section 4.3.1). Therefore, employing in a GUV assay a native acceptor complex could speed up the reaction.

3. Lack of the membrane organisation - The organisation of the proteins reconstituted into GUV membranes can be assumed to be random unless partitioning to membrane domains (Bacia et al., 2004) or clustering (van den Bogaart et al., 2011) occurs. In the reconstitution system utilised in this study it is not the case as can be seen in Figure 3.1 with relatively uniform distribution of fluorescently labelled $\Delta \mathrm{N}$ complex across the GUV membrane. In physiological environment the presynaptic plasma membrane together with adjacent cytoplasm creates a highly organised structure (so called active zone; Südhof, 2012). Therefore, the presynaptic plasma membrane has a very specified protein (Südhof, 2012) and lipid composition (including for example PIP2 — van den Bogaart et al., 2011; PIP3 - Khuong et al., 2013). Also in secretory cells lacking an active zone were detected so called fusion "hotspots" (Yuan et al., 2015). With the specific membrane arrangement cell assures that the time needed for $\mathrm{Ca}^{2+}$ diffusion from the channel to sensor is minimised and that vesicle has sufficient amount of factors (among those available for binding SNARE acceptor complexes) needed to perform fusion. In the GUV assay, liposome or CG after initiation of the first SNARE complex diffuses docked on the GUV surface until the sufficient amount of SNARE complexes needed for fusion are formed. It was previously shown that for fusion of two SUVs one SNARE complex is sufficient (van den Bogaart et al., 2010) but here, due to lower membrane curvature, and 
based on evidence discussed also in Section 4.3, clearly formation of multiple ternary SNARE complexes is needed. This process could be greatly accelerated if the local concentration of acceptor complex would be increased.

4. Lipid composition - Neuronal cells accumulate polyunsaturated fatty acids towards the axonal tip (Yang et al., 2012). This type of lipids was reported to induce lipid packing defects that could lower the energy of membrane deformation (Vamparys et al., 2013) and support endocytosis (Pinot et al., 2014). This can be further supported by finding that some of the polyunsaturated fatty acids speed up vesicle exocytosis in PC12 cells (Ong et al., 2006). Therefore, one could hypothesise that presence of such polyunsaturated lipids in membranes of vesicles used in this study could also facilitate membrane fusion. Other lipid that was shown to be important for exocytosis speed is sphingomyelin. Sphingosine that is produced from this lipid can activate exocytosis (Darios et al., 2009) and therefore could also speed up the fusion in an in vitro assay.

Keeping in mind that lag times between docking and fusion reported by the assay presented in this work can be even further increased, it can be concluded that SNARE proteins, without any cofactors, can catalyse membrane fusion with millisecond kinetics, not only with small vesicles but also with large secretory granules. These findings support the hypothesis that SNARE zippering can be completed in sub-millisecond time after the initial trans-contact. In this situation, there is no need for intermediate states with SNAREs arrested at a partially zippered state that was thought to explain the fast fusion kinetics in neurons (Jahn and Fasshauer, 2012). Initially, the model with partially zippered complexes was developed because only small conformational rearrangements of SNARE proteins would be necessary upon $\mathrm{Ca}^{2+}$ signal. Such SNARE configuration could be achieved by interaction with an accessory protein playing a role of a "clamp" (see also Section 1.2.3) with primary candidates being synaptotagmin (Chicka et al., 2008) or complexin (Kümmel et al., 2011; Yang et al., 2010). The clamp needs to be released rapidly upon the $\mathrm{Ca}^{2+}$ signal in order to allow the SNARE complex to complete zippering. Nevertheless, experimentally long-lived trans-complexes were difficult to capture and mechanism that would maintain the partially zippered state has remained elusive (Jahn and Fasshauer, 2012). Therefore, an alternative model obviating the need of partially zippered SNARE complexes has been proposed (Gerber et al., 2008; Jahn and Fasshauer, 2012). In this model the control of membrane fusion is put upstream of SNARE complex nucleation and it envisions SNARE machinery as "single shot" device that once nucleated translates all the zippering energy 
into membrane fusion. Additional advantage of such arrangement would allow to also displace the accessory proteins upon SNARE zippering initiation avoiding the steric hindrance caused by multiple bulky proteins bound. The evidence presented in this work provides an argument for the second model with SNAREs envisioned as "single shot" devices.

\subsection{The effect of SNARE complex assembly on vesicle docking and membrane fusion}

In this study, it could be observed that syb-LUVs upon docking diffuse on the $\Delta \mathrm{N}$-GUV membrane surface and can then further proceed to either the full fusion or to undock. The diffusion of docked vesicles has not been reported before in other available fusion assays involving planar bilayers (for the overview of in vitro fusion assays see Section 1.3.2) even if the docking times were prolonged ( $>50 \mathrm{~s}$ ) like in the work of Schwenen et al. (2015). Such events were not observed despite the fact that the protein mobility was checked to be normal (Wagner and Tamm, 2001; Schwenen et al., 2015). What is more, vesicle immobilisation in a simple membrane environment consisting of lipids and SNARE-proteins only seems not natural in comparison to relative vesicle immobilisation during docking-fusion process in cells where the molecular crowding as well as cytoskeleton may play a role. Nevertheless, small displacement of exocytotic vesicles after docking was observed in cells before (e.g. for GLUT4 containing vesicles — Li et al., 2004; Yuan et al., 2015; chromaffin granules — Steyer et al., 1997; Steyer and Almers, 1999; Karatekin et al., 2008; synaptic vesicles - Jordan et al., 2005; Lemke and Klingauf, 2005; Park et al., 2012a). However, the molecular nature of membrane attachment is unknown and may not be SNARE-mediated. Probably the only example of diffusive behaviour of a docked vesicle in reconstitution system was reported by Tareste et al. (2008). In the presented there assay the plasma membrane SNARE proteins were located on a small liposomes and synaptobrevin on GUVs (opposite arrangement to the synaptic vesicle-plasma membrane morphology). Nevertheless, the fact that docked liposome diffusion can be observed among in vitro assays only when GUVs are used, points out another advantage of a GUV-based system.

\subsubsection{Reversibility of the SNARE complex assembly}

This study presents that SNARE-mediated interactions of liposomes or granules with GUV membrane can be reversed (Section 3.3.1, Figure 3.19). One of the possible ex- 
planations for this phenomenon is fact that the stabilised SNARE acceptor complex $(\Delta \mathrm{N}$ complex) was used on GUV membrane. This complex has been already shown before to reversibly interact with synaptobrevin during the first N-terminal part of zippering, before the displacement of syb 49-96, in case when a short syb fragment (1-59 or 1-52) was used (Pobbati et al., 2006; Wiederhold and Fasshauer, 2009). In fact, both $\mathrm{N}$ - and C-terminal SNARE complex zippering was shown to be reversible with only the full zippering being quasi-irreversible (Wiederhold et al., 2010). SNARE-mediated interactions of liposomes could be also reversed by competition with a soluble domain of syb (syb 1-96) in case of full length syb containing 2 alanine substitutions in the Nterminal part of the SNARE-motif (I45A, M46A syb, referred here as AA syb; Yavuz, 2015 ) and the $\Delta \mathrm{N}$ complex. Although the $\Delta \mathrm{N}$ complex has certain advantages (see discussion about acceptor complex on page 86 in Section 4.2), the need for displacement of the stabilising syb fragment (49-96) perturbs the zippering process itself, and therefore slows it down, possibly allowing more flexibility during the N-terminal zippering. In a situation with an acceptor complex consisting of one molecule of syntaxin 1 and one of SNAP-25, zippering probably progresses very fast through all layers of the SNARE complex and making the zippering process quasi-irreversible (Fasshauer et al., 2002; Wiederhold and Fasshauer, 2009). On the other hand, it cannot be excluded that in vivo exists a molecular "clamp" preventing C-terminal SNARE complex zippering in a similar way as syb 49-96 in the $\Delta \mathrm{N}$ complex, and/or that accessory proteins (like for example Munc18 - Baker et al., 2015) stabilise the N-terminal assembly (see also discussion about partially zippered SNARE complex in Section 4.2). What is more, undocking was also observed in vivo (e.g. "bounce" events in Midorikawa and Sakaba, 2015), though since the molecular nature of docking is unknown it could not be directly attributed to SNARE complex. In the presented in this study docking and fusion assay, undocking is therefore most probably a result of multiple SNARE complexes assembling and disassembling randomly before the first syb 49-96 is displaced, leading sometimes to situation that no complex is "holding" the vesicle at the GUV membrane any more, and the vesicle can diffuse away (visible in microscopy images as undocking). This hypothesis can be further supported by the finding of weak cooperative coupling between assembling SNARE complexes in fusing LUVs (Hernandez et al., 2014). It is the first to our knowledge demonstration that the $\Delta \mathrm{N}$ complex and WT full length syb can reversibly interact. This reversibility could not be observed in previous studies of interactions between the $\Delta \mathrm{N}$ complex and syb (Wiederhold and Fasshauer, 2009) probably due to absence of membranes. In this study, presence of negatively charged lipids in both membranes (10 mol\% PS) leads to vesicle-GUV repulsion and therefore potentiates the "reversibility effect". 


\subsubsection{Do two vesicle docking modes detected with iSCAT represent inter- mediates of membrane fusion pathway?}

More results concerning the SNARE zippering mechanism came from analysis of the dynamics of docked vesicles with iSCAT microscopy (Section 3.3.2). Observation of syb-LUVs containing either WT or AA syb docking and diffusing on the $\triangle \mathrm{N}$-GUV membrane indicates that there is no single docking mode, but rather there are at least 2 represented by loose and tight docking (Figure 3.22 and 3.23). It has to be mentioned that observation of WT syb-LUVs docking for prolonged times and not fusing was possible due to previous rounds of LUV to GUV fusion that depleted the number of $\Delta \mathrm{N}$ complexes available for SNARE complex formation by either utilising them for fusion reaction itself or by subsequent lateral diffusion of WT syb on the GUV and cis complex formation (observed also previously by Hernandez, 2012). The remaining acceptor complexes were not sufficient to generate energy needed for fusion of LUVs with GUVs. This points out that there is definitely more then one SNARE complex needed for fusion of LUVs with GUVs.

Another question that arises, is why there is significantly less "tightly docked" AA syb-LUVs than WT LUVs (Figure 3.23). The AA syb variant was first used in a study by Wiederhold et al. (2010) where it was suggested that mutated amino acids (I45 and M46) represent a coiling "trigger site" needed for efficient SNARE complex assembly. In the I45A, M46A syb mutant (AA syb) this trigger site is perturbed which leads to drop in small liposome fusion efficiency (Wiederhold et al., 2010; and no fusion in case of LUVs - Yavuz, 2015), and decreased neurotransmitter release in chromaffin cells (Wiederhold et al., 2010). It was also found that AA syb is less efficient in displacing the syb 49-96 fragment from the $\Delta \mathrm{N}$ complex and its binding can be competed out with WT syb 1-96 (Yavuz, 2015). In turn, it leads to a conclusion that AA syb stalls the fusion reaction at earlier stage then $\Delta 84$ syb (Yavuz, 2015) or WT syb in case of limited availability of $\Delta \mathrm{N}$ complexes (Figure 3.23). This block can be sometimes overcome and lead to "tigh docking" probably due to collisions of LUV and GUV increased during loose docking. The fact that loose docking can be also found in WT syb samples indicates that loose docking is an evidence of a transient zippering intermediate rather than a kinetic dead-end.

The question remains what is the molecular state of the tightly and loosely docked vesicles. Since the distinction of the two docking types is made based on the dynamic behaviour description, it could be hard to assign them to electron microscopy data. However, it was shown before that $\Delta 84$ syb-LUVs, as well as AA syb-LUVs with 
$\Delta \mathrm{N}-L U V \mathrm{~s}$, can form a "tightly docked state" (Hernandez et al. $2012-\Delta 84 \mathrm{syb}$, and Dr. Halenur Yavuz, personal communication - AA syb). This structure visible in electron micrographs presents two vesicles with apposed membranes tightly pressed against each other but neither fused nor hemifused (Hernandez, 2012; Hernandez et al., 2012). It was already postulated that interface between these tightly pressed membranes is dehydrated (Hernandez, 2012). Additionally, results from docking FCCS-based assays (Cypionka et al., 2009) point out existence of irreversibly docked vesicles even when trans SNARE complexes are disassembled by NSF (Yavuz, 2015). Moreover, tight and loose trans SNARE complexes were also described in physiological context in chromaffin cells (Xu et al., 1998, 1999). Taking all this into account, it is conceivable that tightly docked vesicles observed with iSCAT microscopy also represent structures visualised previously with electron microscopy (Hernandez et al., 2012). However, it remains to be elucidated whether this docking is insensitive to SNARE complex disassembly like in Yavuz (2015) and whether there is increased in comparison to AA syb number of tightly docked $\Delta 84$ syb-LUVs. At this point it cannot be also excluded that tightly docked vesicles from this study are hemifused to the GUV membrane. Nonetheless, this scenario seems unlikely since neither of discussed here syb mutants ( $\Delta 84$ and AA syb) is able to induce hemifusion of two LUVs (Hernandez et al., 2012 and Dr. Halenur Yavuz, personal communication) that have a higher net curvature (i.e. lower energy barrier for fusion) than a LUV with a GUV together.

The more detailed analysis of the diffusion dynamics of docked vesicles presented in this work revealed that vesicles identified as tightly docked diffuse slower than loosely docked (Figure 3.24). This fact further supports the distinct nature of two described here docking modes. Additionally, the loose state mobilities are much more dynamically heterogeneous than tight ones (as could be inferred from distribution of major and minor diffusion coefficients of AA syb-LUVs, Figure 3.24 E). In a presented here system, the diffusion coefficient probably depends on (i) the adhesion surface between the LUV and GUV membrane lipids (that probably increases with time up to a point when membrane tension of an LUV counteracts its further expansion), and/or (ii) the current number of SNARE complexes engaged in the docking process (what makes the whole process more complex taking into account assembling and disassembling complexes as discussed in Section 4.3.1). If the hypothesis that tightly docked state represents a dehydrated bilayer-bilayer contact sites holds true then (i) would contribute mostly to diffusion coefficients of vesicles docked in a tight mode, while (ii) would be the main determinant of diffusion coefficients of loose vesicles. To prove this scenario computer modelling similar to the one presented by Warner et al. (2009) could be helpful. 
Very puzzling observation of fast minor component of diffusion coefficients corresponding to tightly docked AA syb-LUVs suggests that such vesicles might transiently detach from the GUV while being tightly docked for the remaining majority of time. The diffusional mobility reaching above $5 \mathrm{\mu m}^{2} / \mathrm{s}$ corresponds well with the previously measured values of vesicles diffusing in a planar membrane proximity without docking (Kyoung and Sheets, 2008). This observation may actually contradict the hypothesis of formation of irreversible dehydrated bilayer-bilayer contact sites by AA syb-LUVs with $\Delta \mathrm{N}-\mathrm{GUVs}$. On the other hand, since docking mode in a single track was unambiguously classified as either tight or loose, such vesicles might have been loosely docked for a minor time at the beginning of a track and subsequently developed a tight docking morphology. Here, analysis of more docking events would be necessary to investigate this issue. 


\subsection{Conclusions and Outlook}

In conclusion, experiments presented in this work show:

1. A method for $\triangle \mathrm{N}-\mathrm{GUV}$ formation;

2. A novel single vesicle in vitro fusion assay based on GUVs combined with either liposomes or purified secretory vesicles;

3. That SNAREs only can induce very fast membrane fusion of large vesicles (liposomes or CGs) to GUVs in milliseconds after initial contact and therefore provide an evidence against the need of partially zippered SNARE complexes for sub-millisecond synaptic vesicle fusion events in vivo;

4. That docked vesicles and therefore SNARE proteins can reversibly interact with each other (when the $\Delta \mathrm{N}$ complex and full length syb are used);

5. A label-free method of observation of vesicle docking with iSCAT microscopy in in vitro reconstitution experiments;

6. That fusion of LUVs to GUVs requires more than one SNARE complex; and

7. Two SNARE-mediated vesicle docking modes (loose and tight) that can represent stalled intermediates of SNARE-induced membrane fusion pathway.

Despite the fact that GUV-based fusion assay presented in this work demonstrated membrane fusion events with millisecond kinetics, there is still a number of improvements that can be implemented in order to make the assay more optimal and/or physiological (in addition to factors already discussed on page 86) :

1. Improved GUV formation - As mentioned in Section 4.1 the $\Delta \mathrm{N}-\mathrm{GUV}$ formation protocol can be still improved. In order to make the assay more physiological, a buffer with $\sim 150 \mathrm{mM}$ salt should be included in the GUV lumen. It would be also important to create GUV bilayer assymetry and include other kinds of lipids that were shown to play an essential role in the presynaptic plasma membrane like phosphoinositides (PIP2, Martin, 2012; and PIP3, Khuong et al., 2013), sphingomyelin (Darios et al., 2009), or polyunsaturated fatty acids (Yang et al., 2012). 
2. Improvement of imaging - The temporal resolution of the assay should be improved in order to allow identification of fusion events faster than the current time resolution of $12 \mathrm{~ms}$. In the future, it is possible that even faster scanners than available in the Leica SP8 setup can be developed to improve temporal resolution. From the currently available fluorescence microscopy techniques spinning disc confocal microscopy (like imaging of local $\mathrm{Ca}^{2+}$ influx in hair cells in Ohn et al., 2016) could provide a better alternative for the confocal scanning setup used in this study. Although imaging of fusion events on GUVs with various widefield microscopy setups (also including laser excitation and CMOS cameras) was not successful (data not shown) due to the very low signal-to-noise ratio, it is possible that it could be improved in a custom-build imaging setup. Various tricks could be employed like for example use of cylindrical lens (improving z-resolution; Huang et al., 2008), planar (Holekamp et al., 2008) or line illumination (Junek et al., 2009), with the two latter ones allowing video-rate 3D imaging. Moreover, the assay is currently being further developed for label-free detection of fusion events with iSCAT microscopy.

3. Improved data analysis - Up to this point, detection of docking and fusion events was done manually. A natural next step in the assay development would be to automatise detection of vesicle docking and subsequent automatic monitoring of diffusion on GUV surface, undocking or fusion. This could be done by correlation of vesicle position relative to GUV and subsequent tracking (possibly in all dimensions). By employing filtering methods and peak detection one could automatically extract from GUV membrane line profiles events with vesicle fluorescence intensity peak on GUV membrane followed by peak broadening indicating fusion induced lipid mixing (like on Figure 3.14).

4. Use of accessory proteins - Accessory proteins are known to be essential for regulation of the synaptic vesicle exocytosis (Section 1.2.3). Here, implementation of at least a basic set of accessory proteins (like Munc18, fragments of Munc13, synaptotagmin, NSF and $\alpha$ SNAP - Ma et al., 2013; along with complexin) could help in understanding of the fusion process regulation. In this case also more physiological SNARE acceptor complex should be utilised (as discussed in Section 4.2).

5. Controlled membrane tension - Membrane tension has been shown to be important factor in regulation of endo- and exocytosis (Gauthier et al., 2012). Therefore, adjusting this parameter is crucial to recapitulate synaptic vesicle 
fusion. Recently, it has been shown that simply by modifying the concentration of magnesium ions in solution one can control the GUV adhesion to the coverslip and therefore its membrane tension (Gleisner et al., 2016). Another method to control GUV membrane tension is membrane aspiration with a micropipette (Evans and Rawicz, 1990; Quemeneur et al., 2014).

6. Other SNARE proteins mutants - In order to fully understand the connection between SNARE zippering and membrane fusion more experiments need to be performed including also other SNARE mutants that would allow identification of other intermediate steps of the reaction. One example could be SNAP-25 mutant with mutation in the +5th layer of the SNARE complex (Sørensen et al., 2006 ), in between $\mathrm{AA}$ and $\Delta 84$ syb mutations. The other possibility to study effect of various factors on fusion speed is to use myricetin - a small hydrophobic molecule interfering with SNARE zippering that can be removed by using enzyme laccase (Heo et al., 2016).

Finally, I believe that a GUV-based fusion assay is a versatile method that can be further adapted for investigation of other fusion topologies requiring fusion of highly curved vesicles to relatively flat membrane, for example in other membrane trafficking steps in cells like fusion of vesicles to endosomes, ER or to Golgi apparatus (also SNARE-mediated fusion); or in case of fusion of enveloped viruses to cell membrane (Kamiya et al., 2010). The presented in this work assay can be also adapted for studying fusion of two large membrane structures like for example vacuoles (Nichols et al., 1997), or cells (Aguilar et al., 2013). 


\section{Bibliography}

Aguilar, P. S., Baylies, M. K., Fleissner, A., Helming, L., Inoue, N., Podbilewicz, B., Wang, H. and Wong, M. (2013). Genetic basis of cell-cell fusion mechanisms. Trends in Genetics 29, 427-437.

Aimon, S., Manzi, J., Schmidt, D., Poveda Larrosa, J. A., Bassereau, P. and Toombes, G. E. S. (2011). Functional reconstitution of a voltage-gated potassium channel in giant unilamellar vesicles. PloS one 6, e25529.

Ajouz, B., Berrier, C., Besnard, M., Martinac, B. and Ghazi, A. (2000). Contributions of the Different Extramembranous Domains of the Mechanosensitive Ion Channel MscL to Its Response to Membrane Tension. Journal of Biological Chemistry 275, $1015-1022$.

Akashi, K., Miyata, H., Itoh, H. and Kinosita, K. (1996). Preparation of giant liposomes in physiological conditions and their characterization under an optical microscope. Biophysical Journal $71,3242-3250$.

Allen, T. M., Romans, A. Y., Kercret, H. and Segrest, J. P. (1980). Detergent removal during membrane reconstitution. Biochimica et Biophysica Acta (BBA) - Biomembranes $601,328-342$.

Angelova, M. I. and Dimitrov, D. S. (1986). Liposome electroformation. Faraday Discussions of the Chemical Society 81, 303-311.

Angelova, M. I., Soléau, S., Méléard, P., Faucon, F. and Bothorel, P. (1992). Preparation of giant vesicles by external AC electric fields. Kinetics and applications. In Trends in Colloid and Interface Science VI, (Helm, C., Lösche, M. and Möhwald, H., eds), number 89 in Progress in Colloid \& Polymer Science pp. 127-131. Steinkopff.

Avery, J., Ellis, D. J., Lang, T., Holroyd, P., Riedel, D., Henderson, R. M., Edwardson, J. M. and Jahn, R. (2000). A Cell-Free System for Regulated Exocytosis in Pc12 Cells. The Journal of Cell Biology 148, 317-324.

Bacia, K., Schuette, C. G., Kahya, N., Jahn, R. and Schwille, P. (2004). SNAREs prefer liquid-disordered over "raft" (liquid-ordered) domains when reconstituted into giant unilamellar vesicles. The Journal of biological chemistry 279, 37951-37955. 
Bai, J., Tucker, W. C. and Chapman, E. R. (2004). PIP2 increases the speed of response of synaptotagmin and steers its membrane-penetration activity toward the plasma membrane. Nature structural \& molecular biology 11, 36-44.

Baker, P. F. and Knight, D. E. (1978). Calcium-dependent exocytosis in bovine adrenal medullary cells with leaky plasma membranes. Nature 276, 620-622.

Baker, R. W. and Hughson, F. M. (2016). Chaperoning SNARE assembly and disassembly. Nature Reviews Molecular Cell Biology 17, 465-479.

Baker, R. W., Jeffrey, P. D., Zick, M., Phillips, B. P., Wickner, W. T. and Hughson, F. M. (2015). A direct role for the Sec1/Munc18-family protein Vps33 as a template for SNARE assembly. Science 349, 1111-1114.

Balch, W. E., Dunphy, W. G., Braell, W. A. and Rothman, J. E. (1984). Reconstitution of the transport of protein between successive compartments of the golgi measured by the coupled incorporation of N-acetylglucosamine. Cell 39, 405-416.

Bastiaens, P. and Jovin, T. (1998). Fluorescence resonance energy transfer microscopy. In Cell Biology: A Laboratory Handbook, (Celis, J., ed.), vol. 3, pp. 136-146. Academic Press, New York 2nd edition.

Bertani, G. (1951). The mode of phage liberation by lysogenic Escherichia coli. Journal of Bacteriology 62, 293-300.

Betz, W. J. and Bewick, G. S. (1992). Optical analysis of synaptic vesicle recycling at the frog neuromuscular junction. Science 255, 200-203.

Blijleven, J. S., Boonstra, S., Onck, P. R., van der Giessen, E. and van Oijen, A. M. (2016). Mechanisms of influenza viral membrane fusion. In Seminars in Cell \& Developmental Biology Elsevier. In Press.

Blodgett, K. B. (1935). Films Built by Depositing Successive Monomolecular Layers on a Solid Surface. Journal of the American Chemical Society 5\%, 1007-1022.

Blodgett, K. B. and Langmuir, I. (1937). Built-Up Films of Barium Stearate and Their Optical Properties. Physical Review 51, 964-982.

Bonifacino, J. S. and Glick, B. S. (2004). The Mechanisms of Vesicle Budding and Fusion. Cell 116, 153-166. 
Bowen, M. E., Weninger, K., Brunger, A. T. and Chu, S. (2004). Single Molecule Observation of Liposome-Bilayer Fusion Thermally Induced by Soluble N-Ethyl Maleimide Sensitive-Factor Attachment Protein Receptors (SNAREs). Biophysical Journal 87, 3569-3584.

Bracher, A., Kadlec, J., Betz, H. and Weissenhorn, W. (2002). X-ray Structure of a Neuronal Complexin-SNARE Complex from Squid. Journal of Biological Chemistry 277, 26517-26523.

Brenner, S. (1974). The Genetics of Caenorhabditis Elegans. Genetics 77, 71-94.

Brose, N., Petrenko, A. G., Sudhof, T. C. and Jahn, R. (1992). Synaptotagmin: a calcium sensor on the synaptic vesicle surface. Science 256, 1021-1025.

Brunger, A. T., Cipriano, D. J. and Diao, J. (2015). Towards reconstitution of membrane fusion mediated by SNAREs and other synaptic proteins. Critical Reviews in Biochemistry and Molecular Biology 50, 231-241.

Burkhardt, P., Hattendorf, D. A., Weis, W. I. and Fasshauer, D. (2008). Munc18a controls SNARE assembly through its interaction with the syntaxin N-peptide. The EMBO journal 27, 923-933.

Chen, X., Tomchick, D. R., Kovrigin, E., Araç, D., Machius, M., Südhof, T. C. and Rizo, J. (2002). Three-dimensional structure of the complexin/SNARE complex. Neuron 33, 397-409.

Chernomordik, L. V. and Kozlov, M. M. (2008). Mechanics of membrane fusion. Nature Structural \& Molecular Biology 15, 675-683.

Chiba, M., Miyazaki, M. and Ishiwata, S. (2014). Quantitative Analysis of the Lamellarity of Giant Liposomes Prepared by the Inverted Emulsion Method. Biophysical Journal 10\%, 346-354.

Chicka, M. C., Hui, E., Liu, H. and Chapman, E. R. (2008). Synaptotagmin arrests the SNARE complex before triggering fast, efficient membrane fusion in response to $\mathrm{Ca}^{2+}$. Nature structural \& molecular biology 15, 827-835.

Choi, U. B., Zhao, M., Zhang, Y., Lai, Y. and Brunger, A. T. (2016). Complexin induces a conformational change at the membrane-proximal C-terminal end of the SNARE complex. eLife 5, e16886. 
Chow, R. H., von Rüden, L. and Neher, E. (1992). Delay in vesicle fusion revealed by electrochemical monitoring of single secretory events in adrenal chromaffin cells. Nature 356, 60-63.

Cold Spring Harbor Protocols (2006). Terrific Broth.

Couteaux, R. and Pécot-Dechavassine, M. (1970). Vésicules synaptiques et poches au niveau des «zones actives» de la jonction neuromusculaire. C. R. Hebd. Seances Acad. Sci., Ser. D, Sci. Nat. 271, 2346-2349.

Crane, J. M., Kiessling, V. and Tamm, L. K. (2005). Measuring Lipid Asymmetry in Planar Supported Bilayers by Fluorescence Interference Contrast Microscopy. Langmuir 21, 1377-1388.

Criado, M. and Keller, B. U. (1987). A membrane fusion strategy for single-channel recordings of membranes usually non-accessible to patch-clamp pipette electrodes. FEBS Letters 224, 172-176.

Crowe, J. H., Crowe, L. M., Carpenter, J. F., Rudolph, A. S., Wistrom, C. A., Spargo, B. J. and Anchordoguy, T. J. (1988). Interactions of sugars with membranes. Biochimica et Biophysica Acta (BBA) - Reviews on Biomembranes 947, $367-384$.

Cypionka, A., Stein, A., Hernandez, J. M., Hippchen, H., Jahn, R. and Walla, P. J. (2009). Discrimination between docking and fusion of liposomes reconstituted with neuronal SNARE-proteins using FCS. Proceedings of the National Academy of Sciences $106,18575-18580$.

Darios, F., Wasser, C., Shakirzyanova, A., Giniatullin, A., Goodman, K., MunozBravo, J. L., Raingo, J., Jorgačevski, J., Kreft, M., Zorec, R., Rosa, J. M., Gandia, L., Gutiérrez, L. M., Binz, T., Giniatullin, R., Kavalali, E. T. and Davletov, B. (2009). Sphingosine Facilitates SNARE Complex Assembly and Activates Synaptic Vesicle Exocytosis. Neuron 62, 683-694.

del Castillo, J. and Katz, B. (1954). Quantal components of the end-plate potential. The Journal of Physiology 124, 560-573.

Dezi, M., Cicco, A. D., Bassereau, P. and Lévy, D. (2013). Detergent-mediated incorporation of transmembrane proteins in giant unilamellar vesicles with controlled physiological contents. Proceedings of the National Academy of Sciences 110, 72767281. 
Diao, J., Grob, P., Cipriano, D. J., Kyoung, M., Zhang, Y., Shah, S., Nguyen, A., Padolina, M., Srivastava, A., Vrljic, M., Shah, A., Nogales, E., Chu, S. and Brunger, A. T. (2012). Synaptic proteins promote calcium-triggered fast transition from point contact to full fusion. eLife 1, e00109.

Diao, J., Su, Z., Ishitsuka, Y., Lu, B., Lee, K. S., Lai, Y., Shin, Y.-K. and Ha, T. (2010). A single-vesicle content mixing assay for SNARE-mediated membrane fusion. Nature Communications 1,54.

Doeven, M. K., Folgering, J. H., Krasnikov, V., Geertsma, E. R., van den Bogaart, G. and Poolman, B. (2005). Distribution, Lateral Mobility and Function of Membrane Proteins Incorporated into Giant Unilamellar Vesicles. Biophysical Journal 88, 11341142.

Domanska, M. K., Kiessling, V., Stein, A., Fasshauer, D. and Tamm, L. K. (2009). Single vesicle millisecond fusion kinetics reveals number of SNARE complexes optimal for fast SNARE-mediated membrane fusion. The Journal of biological chemistry 284, 32158-32166.

Dower, W. J., Miller, J. F. and Ragsdale, C. W. (1988). High efficiency transformation of E. coli by high voltage electroporation. Nucleic Acids Research 16, 6127-6145.

Dulubova, I., Sugita, S., Hill, S., Hosaka, M., Fernandez, I., Südhof, T. C. and Rizo, J. (1999). A conformational switch in syntaxin during exocytosis: role of munc18. The EMBO Journal 18, 4372-4382.

Düzgüneş, N. (2003). Preparation and Quantitation of Small Unilamellar Liposomes and Large Unilamellar Reverse-Phase Evaporation Liposomes. In Liposomes, Part A, (Düzgüneş, N., ed.), vol. 367, of Methods in Enzymology pp. 23-27. Academic Press.

Eaton, J. W., Bateman, D., Hauberg, S. and Wehbring, R. (2015). GNU Octave version 4.0.0 manual: a high-level interactive language for $\mathrm{n}$ umerical computations.

Eccles, J. C., Katz, B. and Kuffler, S. W. (1941). Nature of the "endplate potential" in curarized muscle. Journal of Neurophysiology 4, 362-387.

Evans, E. and Rawicz, W. (1990). Entropy-driven tension and bending elasticity in condensed-fluid membranes. Physical Review Letters 64, 2094-2097. 
Fasshauer, D., Antonin, W., Margittai, M., Pabst, S. and Jahn, R. (1999). Mixed and Non-cognate SNARE Complexes. Characterization of assembly and biophysical properties. Journal of Biological Chemistry 274, 15440-15446.

Fasshauer, D., Antonin, W., Subramaniam, V. and Jahn, R. (2002). SNARE assembly and disassembly exhibit a pronounced hysteresis. Nature Structural \& Molecular Biology 9, 144-151.

Fasshauer, D., Bruns, D., Shen, B., Jahn, R. and Brünger, A. T. (1997a). A Structural Change Occurs upon Binding of Syntaxin to SNAP-25. Journal of Biological Chemistry 272, 4582-4590.

Fasshauer, D., Otto, H., Eliason, W. K., Jahn, R. and Brünger, A. T. (1997b). Structural Changes Are Associated with Soluble N-Ethylmaleimide-sensitive Fusion Protein Attachment Protein Receptor Complex Formation. Journal of Biological Chemistry 272, 28036-28041.

Fasshauer, D., Sutton, R. B., Brunger, A. T. and Jahn, R. (1998). Conserved structural features of the synaptic fusion complex: SNARE proteins reclassified as Q- and RSNAREs. Proceedings of the National Academy of Sciences of the United States of America 95, 15781-15786.

Fix, M., Melia, T. J., Jaiswal, J. K., Rappoport, J. Z., You, D., Söllner, T. H., Rothman, J. E. and Simon, S. M. (2004). Imaging single membrane fusion events mediated by SNARE proteins. Proceedings of the National Academy of Sciences of the United States of America 101, 7311-7316.

Förster, T. (1948). Zwischenmolekulare Energiewanderung und Fluoreszenz. Annalen der Physik 437, 55-75.

Galush, W. J., Nye, J. A. and Groves, J. T. (2008). Quantitative Fluorescence Microscopy Using Supported Lipid Bilayer Standards. Biophysical Journal 95, 25122519.

Gasteiger, E., Hoogland, C., Gattiker, A., Duvaud, S., Wilkins, M., Appel, R. and Bairoch, A. (2005). Protein Identification and Analysis Tools on the ExPASy Server. In The Proteomics Protocols Handbook, (Walker, J., ed.), pp. 571-607. Humana Press.

Gauthier, N. C., Masters, T. A. and Sheetz, M. P. (2012). Mechanical feedback between membrane tension and dynamics. Trends in Cell Biology 22, 527-535. 
Gerber, S. H., Rah, J.-C., Min, S.-W., Liu, X., Wit, H. d., Dulubova, I., Meyer, A. C., Rizo, J., Arancillo, M., Hammer, R. E., Verhage, M., Rosenmund, C. and Südhof, T. C. (2008). Conformational Switch of Syntaxin-1 Controls Synaptic Vesicle Fusion. Science 321, 1507-1510.

Gibson, G. A. and Loew, L. M. (1979). Phospholipid vesicle fusion monitored by fluorescence energy transfer. Biochemical and Biophysical Research Communications $88,135-140$.

Girard, P., Pécréaux, J., Lenoir, G., Falson, P., Rigaud, J.-L. and Bassereau, P. (2004). A New Method for the Reconstitution of Membrane Proteins into Giant Unilamellar Vesicles. Biophysical Journal 8\%, 419-429.

Gleisner, M., Kroppen, B., Fricke, C., Teske, N., Kliesch, T.-T., Janshoff, A., Meinecke, M. and Steinem, C. (2016). Epsin N-terminal homology domain (ENTH) activity as a function of membrane tension. Journal of Biological Chemistry.

Greene, L. A. and Tischler, A. S. (1976). Establishment of a noradrenergic clonal line of rat adrenal pheochromocytoma cells which respond to nerve growth factor. Proceedings of the National Academy of Sciences of the United States of America 73, 2424-2428.

Hallermann, S., Fejtova, A., Schmidt, H., Weyhersmüller, A., Silver, R. A., Gundelfinger, E. D. and Eilers, J. (2010). Bassoon Speeds Vesicle Reloading at a Central Excitatory Synapse. Neuron 68, 710-723.

Hanson, P. I., Roth, R., Morisaki, H., Jahn, R. and Heuser, J. E. (1997). Structure and Conformational Changes in NSF and Its Membrane Receptor Complexes Visualized by Quick-Freeze/Deep-Etch Electron Microscopy. Cell 90, 523-535.

Hayashi, T., McMahon, H., Yamasaki, S., Binz, T., Hata, Y., Südhof, T. C. and Niemann, H. (1994). Synaptic vesicle membrane fusion complex: action of clostridial neurotoxins on assembly. The EMBO Journal 13, 5051-5061.

Hazzard, J., Südhof, T. C. and Rizo, J. (1999). NMR analysis of the structure of synaptobrevin and of its interaction with syntaxin. Journal of Biomolecular NMR $14,203-207$.

Helfrich, W. (1973). Elastic properties of lipid bilayers: theory and possible experiments. Zeitschrift Für Naturforschung. Teil C: Biochemie, Biophysik, Biologie, Virologie 28, 693-703. 
Heo, P., Yang, Y., Han, K. Y., Kong, B., Shin, J.-H., Jung, Y., Jeong, C., Shin, J., Shin, Y.-K., Ha, T. and Kweon, D.-H. (2016). A Chemical Controller of SNARE-Driven Membrane Fusion That Primes Vesicles for $\mathrm{Ca}^{2+}$-Triggered Millisecond Exocytosis. Journal of the American Chemical Society 138, 4512-4521.

Hernandez, J. M. (2012). Reconstituted SNARE-mediated fusion: towards a mechanistic understanding. PhD thesis, Göttingen Graduate School for Neurosciences, Biophysics, and Molecular Biosciences.

Hernandez, J. M., Kreutzberger, A. J. B., Kiessling, V., Tamm, L. K. and Jahn, R. (2014). Variable cooperativity in SNARE-mediated membrane fusion. Proceedings of the National Academy of Sciences 111, 12037-12042.

Hernandez, J. M., Stein, A., Behrmann, E., Riedel, D., Cypionka, A., Farsi, Z., Walla, P. J., Raunser, S. and Jahn, R. (2012). Membrane fusion intermediates via directional and full assembly of the SNARE complex. Science (New York, N.Y.) 336, 1581-1584.

Heuser, J. E. and Reese, T. S. (1981). Structural changes after transmitter release at the frog neuromuscular junction. The Journal of Cell Biology 88, 564-580.

Heuser, J. E., Reese, T. S., Dennis, M. J., Jan, Y., Jan, L. and Evans, L. (1979). Synaptic vesicle exocytosis captured by quick freezing and correlated with quantal transmitter release. The Journal of Cell Biology 81, 275-300.

Höfer, I. and Steinem, C. (2011). A membrane fusion assay based on pore-spanning lipid bilayers. Soft Matter 7, 1644-1647.

Holekamp, T. F., Turaga, D. and Holy, T. E. (2008). Fast three-dimensional fluorescence imaging of activity in neural populations by objective-coupled planar illumination microscopy. Neuron 5\%, 661-672.

Hsieh, C.-L., Spindler, S., Ehrig, J. and Sandoghdar, V. (2014). Tracking Single Particles on Supported Lipid Membranes: Multimobility Diffusion and Nanoscopic Confinement. The Journal of Physical Chemistry B 118, 1545-1554.

Hu, C., Ahmed, M., Melia, T. J., Söllner, T. H., Mayer, T. and Rothman, J. E. (2003). Fusion of Cells by Flipped SNAREs. Science 300, 1745-1749.

Huang, B., Wang, W., Bates, M. and Zhuang, X. (2008). Three-Dimensional SuperResolution Imaging by Stochastic Optical Reconstruction Microscopy. Science 319, 810-813. 
Huber, T. and Sakmar, T. P. (2011). Escaping the flatlands: new approaches for studying the dynamic assembly and activation of GPCR signaling complexes. Trends in Pharmacological Sciences 32, 410-419.

Hui, E., Johnson, C. P., Yao, J., Dunning, F. M. and Chapman, E. R. (2009). Synaptotagmin-Mediated Bending of the Target Membrane Is a Critical Step in $\mathrm{Ca}^{2+}$-Regulated Fusion. Cell 138, 709-721.

Ingolia, T. D. and Koshland, D. E. (1978). The role of calcium in fusion of artificial vesicles. Journal of Biological Chemistry 253, 3821-3829.

Jahn, R. (2008). Some classic papers in the field of membrane fusion - a personal view. Nature Structural \& Molecular Biology 15, 655-657.

Jahn, R. and Fasshauer, D. (2012). Molecular machines governing exocytosis of synaptic vesicles. Nature 490, 201-207.

Jahn, R. and Scheller, R. H. (2006). SNAREs-engines for membrane fusion. Nature reviews. Molecular cell biology 7, 631-643.

Jares-Erijman, E. A. and Jovin, T. M. (2003). FRET imaging. Nature Biotechnology 21, 1387-1395.

Jordan, R., Lemke, E. A. and Klingauf, J. (2005). Visualization of Synaptic Vesicle Movement in Intact Synaptic Boutons Using Fluorescence Fluctuation Spectroscopy. Biophysical Journal 89, 2091-2102.

Junek, S., Chen, T.-W., Alevra, M. and Schild, D. (2009). Activity Correlation Imaging: Visualizing Function and Structure of Neuronal Populations. Biophysical Journal 96, 3801-3809.

Kalb, E., Frey, S. and Tamm, L. K. (1992). Formation of supported planar bilayers by fusion of vesicles to supported phospholipid monolayers. Biochimica et Biophysica Acta (BBA) — Biomembranes 1103, 307-316.

Kamiya, K., Kobayashi, J., Yoshimura, T. and Tsumoto, K. (2010). Confocal microscopic observation of fusion between baculovirus budded virus envelopes and single giant unilamellar vesicles. Biochimica et Biophysica Acta (BBA) - Biomembranes $1798,1625-1631$.

Karatekin, E., Di Giovanni, J., Iborra, C., Coleman, J., O'Shaughnessy, B., Seagar, M. and Rothman, J. E. (2010). A fast, single-vesicle fusion assay mimics physiological 
SNARE requirements. Proceedings of the National Academy of Sciences of the United States of America 107, 3517-3521.

Karatekin, E., Tran, V. S., Huet, S., Fanget, I., Cribier, S. and Henry, J.-P. (2008). A 20-nm Step toward the Cell Membrane Preceding Exocytosis May Correspond to Docking of Tethered Granules. Biophysical Journal 94, 2891-2905.

Kasai, H., Takahashi, N. and Tokumaru, H. (2012). Distinct initial SNARE configurations underlying the diversity of exocytosis. Physiological reviews 92, 1915-1964.

Katz, B. (1969). The Release of neural transmitter substances. Number 10 in The Sherrington lectures / The University of Liverpool, ISSN 0962-5461; ZDB-ID: 9872322, Univ. Pr., Liverpool.

Katz, B. and Miledi, R. (1967). The timing of calcium action during neuromuscular transmission. The Journal of Physiology 189, 535-544.

Kaufmann, A., Beier, V., Franquelim, H. G. and Wollert, T. (2014). Molecular Mechanism of Autophagic Membrane-Scaffold Assembly and Disassembly. Cell 156, 469481.

Keller, B. U., Hedrich, R., Vaz, W. L. C. and Criado, M. (1988). Single channel recordings of reconstituted ion channel proteins: an improved technique. Pflügers Archiv 411, 94-100.

Khuong, T. M., Habets, R. L., Kuenen, S., Witkowska, A., Kasprowicz, J., Swerts, J., Jahn, R., van den Bogaart, G. and Verstreken, P. (2013). Synaptic PI(3,4,5)P3 Is Required for Syntaxin1A Clustering and Neurotransmitter Release. Neuron $7{ }^{7}$, 1097-1108.

Kiessling, V., Ahmed, S., Domanska, M. K., Holt, M. G., Jahn, R. and Tamm, L. K. (2013). Rapid Fusion of Synaptic Vesicles with Reconstituted Target SNARE Membranes. Biophysical Journal 104, 1950-1958.

Kim, J.-Y., Choi, B.-K., Choi, M.-G., Kim, S.-A., Lai, Y., Shin, Y.-K. and Lee, N. K. (2012). Solution single-vesicle assay reveals PIP2-mediated sequential actions of synaptotagmin-1 on SNAREs. The EMBO journal 31, 2144-2155.

Kloepper, T. H., Kienle, C. N. and Fasshauer, D. (2007). An Elaborate Classification of SNARE Proteins Sheds Light on the Conservation of the Eukaryotic Endomembrane System. Molecular Biology of the Cell 18, 3463-3471. 
Kozlov, M. M. and Markin, V. S. (1983). [Possible mechanism of membrane fusion]. Biofizika 28, 242-247.

Kozlov, M. M., McMahon, H. T. and Chernomordik, L. V. (2010). Protein-driven membrane stresses in fusion and fission. Trends in Biochemical Sciences 35, 699706.

Krishnan, M., Mojarad, N., Kukura, P. and Sandoghdar, V. (2010). Geometry-induced electrostatic trapping of nanometric objects in a fluid. Nature 467, 692-695.

Kukura, P., Ewers, H., Müller, C., Renn, A., Helenius, A. and Sandoghdar, V. (2009). High-speed nanoscopic tracking of the position and orientation of a single virus. Nature Methods 6, 923-927.

Kümmel, D., Krishnakumar, S. S., Radoff, D. T., Li, F., Giraudo, C. G., Pincet, F., Rothman, J. E. and Reinisch, K. M. (2011). Complexin cross-links prefusion SNAREs into a zigzag array. Nature Structural \& Molecular Biology 18, 927-933.

Kurps, J., Broeke, J. H., Cijsouw, T., Kompatscher, A., van Weering, J. R. T. and de Wit, H. (2014). Quantitative image analysis tool to study the plasma membrane localization of proteins and cortical actin in neuroendocrine cells. Journal of Neuroscience Methods 236, 1-10.

Kyoung, M. and Sheets, E. D. (2008). Vesicle Diffusion Close to a Membrane: Intermembrane Interactions Measured with Fluorescence Correlation Spectroscopy. Biophysical Journal 95, 5789-5797.

Kyoung, M., Srivastava, A., Zhang, Y., Diao, J., Vrljic, M., Grob, P., Nogales, E., Chu, S. and Brunger, A. T. (2011). In vitro system capable of differentiating fast $\mathrm{Ca}^{2+}$ triggered content mixing from lipid exchange for mechanistic studies of neurotransmitter release. Proceedings of the National Academy of Sciences 108, E304-E313.

Kyoung, M., Zhang, Y., Diao, J., Chu, S. and Brunger, A. T. (2013). Studying calciumtriggered vesicle fusion in a single vesicle-vesicle content and lipid-mixing system. Nature Protocols 8, 1-16.

Lai, Y., Diao, J., Cipriano, D. J., Zhang, Y., Pfuetzner, R. A., Padolina, M. S. and Brunger, A. T. (2014). Complexin inhibits spontaneous release and synchronizes $\mathrm{Ca}^{2+}$-triggered synaptic vesicle fusion by distinct mechanisms. eLife 3, e03756.

Lakowicz, J. R., ed. (2006). Principles of Fluorescence Spectroscopy. Springer Nature. 
Lang, T., Bruns, D., Wenzel, D., Riedel, D., Holroyd, P., Thiele, C. and Jahn, R. (2001). SNAREs are concentrated in cholesterol-dependent clusters that define docking and fusion sites for exocytosis. The EMBO Journal 20, 2202-2213.

Lemke, E. A. and Klingauf, J. (2005). Single Synaptic Vesicle Tracking in Individual Hippocampal Boutons at Rest and during Synaptic Activity. The Journal of Neuroscience 25, 11034-11044.

Leszczyszyn, D. J., Jankowski, J. A., Viveros, O. H., Diliberto, E. J., Near, J. A. and Wightman, R. M. (1990). Nicotinic receptor-mediated catecholamine secretion from individual chromaffin cells. Chemical evidence for exocytosis. Journal of Biological Chemistry 265, 14736-14737.

Li, C. H., Bai, L., Li, D. D., Xia, S. and Xu, T. (2004). Dynamic tracking and mobility analysis of single GLUT4 storage vesicle in live 3T3-L1 cells. Cell Research 14, 480-486.

Lin, R. C. and Scheller, R. H. (1997). Structural Organization of the Synaptic Exocytosis Core Complex. Neuron 19, 1087-1094.

Lindfors, K., Kalkbrenner, T., Stoller, P. and Sandoghdar, V. (2004). Detection and Spectroscopy of Gold Nanoparticles Using Supercontinuum White Light Confocal Microscopy. Physical Review Letters 93, 037401.

Lira, R. B., Dimova, R. and Riske, K. A. (2014). Giant Unilamellar Vesicles Formed by Hybrid Films of Agarose and Lipids Display Altered Mechanical Properties. Biophysical Journal 10\%, 1609-1619.

Ma, C., Su, L., Seven, A. B., Xu, Y. and Rizo, J. (2013). Reconstitution of the Vital Functions of Munc18 and Munc13 in Neurotransmitter Release. Science 339, 421-425.

Malsam, J., Parisotto, D., Bharat, T. A. M., Scheutzow, A., Krause, J. M., Briggs, J. A. G. and Söllner, T. H. (2012). Complexin arrests a pool of docked vesicles for fast $\mathrm{Ca}^{2+}$-dependent release. The EMBO journal 31, 3270-3281.

Manneville, J.-B., Bassereau, P., Lévy, D. and Prost, J. (1999). Activity of Transmembrane Proteins Induces Magnification of Shape Fluctuations of Lipid Membranes. Physical Review Letters 82, 4356-4359. 
Margittai, M., Fasshauer, D., Pabst, S., Jahn, R. and Langen, R. (2001). Homo- and Heterooligomeric SNARE Complexes Studied by Site-directed Spin Labeling. Journal of Biological Chemistry 276, 13169-13177.

Martin, T. F. J. (2012). Role of $\mathrm{PI}(4,5) \mathrm{P}(2)$ in Vesicle Exocytosis and Membrane Fusion. Sub-Cellular Biochemistry 59, 111-130.

Mayer, A., Wickner, W. and Haas, A. (1996). Sec18p (NSF)-Driven Release of Sec17p $(\alpha-$ SNAP) Can Precede Docking and Fusion of Yeast Vacuoles. Cell 85, 83-94.

McIntyre, J. C. and Sleight, R. G. (1991). Fluorescence assay for phospholipid membrane asymmetry. Biochemistry 30, 11819-11827.

McPhee, C., Zoriniants, G., Langbein, W. and Borri, P. (2013). Measuring the Lamellarity of Giant Lipid Vesicles with Differential Interference Contrast Microscopy. Biophysical Journal 105, 1414-1420.

Méléard, P., Bagatolli, L. A. and Pott, T. (2009). Chapter 9 - Giant Unilamellar Vesicle Electroformation: From Lipid Mixtures to Native Membranes Under Physiological Conditions. In Methods in Enzymology vol. 465, of Methods in Enzymology pp. 161 - 176. Academic Press.

Midorikawa, M. and Sakaba, T. (2015). Imaging Exocytosis of Single Synaptic Vesicles at a Fast CNS Presynaptic Terminal. Neuron 88, 492-498.

Miesenböck, G., De Angelis, D. A. and Rothman, J. E. (1998). Visualizing secretion and synaptic transmission with pH-sensitive green fluorescent proteins. Nature 394, 192-195.

Misura, K. M. S., Scheller, R. H. and Weis, W. I. (2000). Three-dimensional structure of the neuronal-Sec1-syntaxin 1a complex. Nature 404, 355-362.

Miura, K. (2012). Analysis of FRAP Curves. European Advanced Light Microscopy Network.

Molecular Probes (2006). Thiol-Reactive Probes. invitrogen mp 00003 edition.

Motta, I., Gohlke, A., Adrien, V., Li, F., Gardavot, H., Rothman, J. E. and Pincet, F. (2015). Formation of Giant Unilamellar Proteo-Liposomes by Osmotic Shock. Langmuir 31, 7091-7099. 
Neher, E. and Marty, A. (1982). Discrete changes of cell membrane capacitance observed under conditions of enhanced secretion in bovine adrenal chromaffin cells. Proceedings of the National Academy of Sciences of the United States of America $79,6712-6716$.

Neher, E. and Sakaba, T. (2008). Multiple Roles of Calcium Ions in the Regulation of Neurotransmitter Release. Neuron 59, 861-872.

Nichols, B. J., Ungermann, C., Pelham, H. R., Wickner, W. T. and Haas, A. (1997). Homotypic vacuolar fusion mediated by t- and v-SNAREs. Nature 38\%, 199-202.

Nickel, W., Weber, T., McNew, J. A., Parlati, F., Söllner, T. H. and Rothman, J. E. (1999). Content mixing and membrane integrity during membrane fusion driven by pairing of isolated v-SNAREs and t-SNAREs. Proceedings of the National Academy of Sciences 96, 12571-12576.

Nikolaus, J., Stöckl, M., Langosch, D., Volkmer, R. and Herrmann, A. (2010). Direct Visualization of Large and Protein-Free Hemifusion Diaphragms. Biophysical Journal 98, 1192-1199.

Niles, W. D. and Cohen, F. S. (1987). Video fluorescence microscopy studies of phospholipid vesicle fusion with a planar phospholipid membrane. Nature of membranemembrane interactions and detection of release of contents. The Journal of General Physiology 90, 703-735.

Nosov, G., Glyvuk, N., Tsytsyura, Y., Trahe, J., Keller, U., Reissner, C., Piehler, J., Missler, M. and Klingauf, J. (2016). Structure of Xenapses - Pure Presynaptic Boutons Induced on Micropatterned Host Substrates. Biophysical Journal 110, 429a.

Novagen (2003). pET System Manual. TB055 10th Edition Rev.B 0403.

Novick, P., Field, C. and Schekman, R. (1980). Identification of 23 complementation groups required for post-translational events in the yeast secretory pathway. Cell 21, 205-215.

Ohn, T.-L., Rutherford, M. A., Jing, Z., Jung, S., Duque-Afonso, C. J., Hoch, G., Picher, M. M., Scharinger, A., Strenzke, N. and Moser, T. (2016). Hair cells use active zones with different voltage dependence of $\mathrm{Ca}^{2+}$ influx to decompose sounds into complementary neural codes. Proceedings of the National Academy of Sciences of the United States of America 113, E4716-4725. 
Ong, W.-L., Jiang, B., Tang, N., Ling, S.-F., Yeo, J.-F., Wei, S., Farooqui, A. A. and Ong, W.-Y. (2006). Differential Effects of Polyunsaturated Fatty Acids on Membrane Capacitance and Exocytosis in Rat Pheochromocytoma-12 Cells. Neurochemical Research 31, 41-48.

Ortega-Arroyo, J. and Kukura, P. (2012). Interferometric scattering microscopy (iSCAT): new frontiers in ultrafast and ultrasensitive optical microscopy. Physical Chemistry Chemical Physics 14, 15625-15636.

Pabst, S. (2002). Studien zur Interaktion von Complexin mit dem synaptischen SNARE-Komplex. PhD thesis, Göttingen.

Palay, S. L. and Palade, G. E. (1955). The fine structure of neurons. The Journal of Biophysical and Biochemical Cytology 1, 69-88.

Park, H., Li, Y. and Tsien, R. W. (2012a). Influence of Synaptic Vesicle Position on Release Probability and Exocytotic Fusion Mode. Science 335, 1362-1366.

Park, Y., Hernandez, J. M., van den Bogaart, G., Ahmed, S., Holt, M., Riedel, D. and Jahn, R. (2012b). Controlling synaptotagmin activity by electrostatic screening. Nat Struct Mol Biol 19, 991-997.

Park, Y., Seo, J. B., Fraind, A., Pérez-Lara, A., Yavuz, H., Han, K., Jung, S.-R., Kattan, I., Walla, P. J., Choi, M., Cafiso, D. S., Koh, D.-S. and Jahn, R. (2015). Synaptotagmin-1 binds to PIP2-containing membrane but not to SNAREs at physiological ionic strength. Nature Structural \& Molecular Biology 22, 815-823.

Pierce Biotechnology (2013). Pierce 660nm Protein Assay. ThermoFisher Scientific 2070.3 edition.

Pincet, F., Adrien, V., Yang, R., Delacotte, J., Rothman, J. E., Urbach, W. and Tareste, D. (2016). FRAP to Characterize Molecular Diffusion and Interaction in Various Membrane Environments. PLOS ONE 11, e0158457.

Pinot, M., Vanni, S., Pagnotta, S., Lacas-Gervais, S., Payet, L.-A., Ferreira, T., Gautier, R., Goud, B., Antonny, B. and Barelli, H. (2014). Polyunsaturated phospholipids facilitate membrane deformation and fission by endocytic proteins. Science 345, 693-697.

Pobbati, A. V., Stein, A. and Fasshauer, D. (2006). N- to C-terminal SNARE complex assembly promotes rapid membrane fusion. Science (New York, N.Y.) 313, 673-676. 
Poo, M.-m. and Cone, R. A. (1973). Lateral diffusion of rhodopsin in the visual receptor membrane. Journal of Supramolecular Structure 1, 354.

Poo, M.-m. and Cone, R. A. (1974). Lateral diffusion of rhodopsin in the photoreceptor membrane. Nature 247, 438-441.

Pott, T., Bouvrais, H. and Méléard, P. (2008). Giant unilamellar vesicle formation under physiologically relevant conditions. Chemistry and physics of lipids $154,115-$ 119.

Quemeneur, F., Sigurdsson, J. K., Renner, M., Atzberger, P. J., Bassereau, P. and Lacoste, D. (2014). Shape matters in protein mobility within membranes. Proceedings of the National Academy of Sciences 111, 5083-5087.

Rand, R. P. (1981). Interacting Phospholipid Bilayers: Measured Forces and Induced Structural Changes. Annual Review of Biophysics and Bioengineering 10, 277-314.

Reeves, J. P. and Dowben, R. M. (1969). Formation and properties of thin-walled phospholipid vesicles. Journal of Cellular Physiology 73, 49-60.

Richmond, D. L., Schmid, E. M., Martens, S., Stachowiak, J. C., Liska, N. and Fletcher, D. A. (2011). Forming giant vesicles with controlled membrane composition, asymmetry, and contents. Proceedings of the National Academy of Sciences of the United States of America 108, 9431-9436.

Richmond, J. E., Weimer, R. M. and Jorgensen, E. M. (2001). An open form of syntaxin bypasses the requirement for UNC-13 in vesicle priming. Nature 412, 338-341.

Rigaud, J.-L. and Lévy, D. (2003). Reconstitution of Membrane Proteins into Liposomes. In Methods in Enzymology, (Nejat Duzgunes, ed.), vol. Volume 372, of Liposomes, Part B pp. 65-86. Academic Press.

Risselada, H. J. and Grubmüller, H. (2012). How SNARE molecules mediate membrane fusion: recent insights from molecular simulations. Current opinion in structural biology 22, 187-196.

Risselada, H. J., Kutzner, C. and Grubmüller, H. (2011). Caught in the act: visualization of SNARE-mediated fusion events in molecular detail. Chembiochem: a European journal of chemical biology 12, 1049-1055. 
Ritzau-Jost, A., Delvendahl, I., Rings, A., Byczkowicz, N., Harada, H., Shigemoto, R., Hirrlinger, J., Eilers, J. and Hallermann, S. (2014). Ultrafast Action Potentials Mediate Kilohertz Signaling at a Central Synapse. Neuron 84, 152-163.

Rizo, J., Chen, X. and Araç, D. (2006). Unraveling the mechanisms of synaptotagmin and SNARE function in neurotransmitter release. Trends in Cell Biology 16, 339350.

Sabatini, B. L. and Regehr, W. G. (1996). Timing of neurotransmission at fast synapses in the mammalian brain. Nature 384, 170-172.

Schägger, H. and von Jagow, G. (1987). Tricine-sodium dodecyl sulfate-polyacrylamide gel electrophoresis for the separation of proteins in the range from 1 to $100 \mathrm{kDa}$. Analytical Biochemistry 166, 368-379.

Schindelin, J., Arganda-Carreras, I., Frise, E., Kaynig, V., Longair, M., Pietzsch, T., Preibisch, S., Rueden, C., Saalfeld, S., Schmid, B., Tinevez, J.-Y., White, D. J., Hartenstein, V., Eliceiri, K., Tomancak, P. and Cardona, A. (2012). Fiji: an opensource platform for biological-image analysis. Nature Methods 9, 676-682.

Schuette, C. G., Hatsuzawa, K., Margittai, M., Stein, A., Riedel, D., Küster, P., König, M., Seidel, C. and Jahn, R. (2004). Determinants of liposome fusion mediated by synaptic SNARE proteins. Proceedings of the National Academy of Sciences of the United States of America 101, 2858-2863.

Schwenen, L. L. G., Hubrich, R., Milovanovic, D., Geil, B., Yang, J., Kros, A., Jahn, R. and Steinem, C. (2015). Resolving single membrane fusion events on planar porespanning membranes. Scientific Reports 5, 12006.

Siddiqui, T. J., Vites, O., Stein, A., Heintzmann, R., Jahn, R. and Fasshauer, D. (2007). Determinants of Synaptobrevin Regulation in Membranes. Molecular Biology of the Cell 18, 2037-2046.

Smith, A. D. and Winkler, H. (1967). A simple method for the isolation of adrenal chromaffin granules on a large scale. Biochemical Journal 103, 480-482.

Smith, E. A. and Weisshaar, J. C. (2011). Docking, Not Fusion, as the Rate-Limiting Step in a SNARE-Driven Vesicle Fusion Assay. Biophysical Journal 100, 2141-2150.

Söllner, T., Bennett, M. K., Whiteheart, S. W., Scheller, R. H. and Rothman, J. E. (1993a). A protein assembly-disassembly pathway in vitro that may correspond to sequential steps of synaptic vesicle docking, activation, and fusion. Cell 75, 409-418. 
Söllner, T., Whiteheart, S. W., Brunner, M., Erdjument-Bromage, H., Geromanos, S., Tempst, P. and Rothman, J. E. (1993b). SNAP receptors implicated in vesicle targeting and fusion. Nature 362, 318-324.

Sørensen, J. B., Wiederhold, K., Müller, E. M., Milosevic, I., Nagy, G., de Groot, B. L., Grubmüller, H. and Fasshauer, D. (2006). Sequential N- to C-terminal SNARE complex assembly drives priming and fusion of secretory vesicles. The EMBO Journal 25, 955-966.

Soumpasis, D. M. (1983). Theoretical analysis of fluorescence photobleaching recovery experiments. Biophysical Journal 41, 95-97.

Stein, A., Weber, G., Wahl, M. C. and Jahn, R. (2009). Helical extension of the neuronal SNARE complex into the membrane. Nature $460,525-528$.

Stevens, D. R., Schirra, C., Becherer, U. and Rettig, J. (2011). Vesicle Pools: Lessons from Adrenal Chromaffin Cells. Frontiers in Synaptic Neuroscience 3, 2.

Steyer, J. A. and Almers, W. (1999). Tracking Single Secretory Granules in Live Chromaffin Cells by Evanescent-Field Fluorescence Microscopy. Biophysical Journal $76,2262-2271$.

Steyer, J. A., Horstmann, H. and Almers, W. (1997). Transport, docking and exocytosis of single secretory granules in live chromaffin cells. Nature 388, 474-478.

Struck, D. K., Hoekstra, D. and Pagano, R. E. (1981). Use of resonance energy transfer to monitor membrane fusion. Biochemistry 20, 4093-4099.

Südhof, T. C. (2012). The presynaptic active zone. Neuron 75, 11-25.

Südhof, T. C. (2013). Neurotransmitter Release: The Last Millisecond in the Life of a Synaptic Vesicle. Neuron 80, 675-690.

Sutton, R. B., Fasshauer, D., Jahn, R. and Brunger, A. T. (1998). Crystal structure of a SNARE complex involved in synaptic exocytosis at 2.4 A resolution. Nature 395, $347-353$.

Takamori, S., Holt, M., Stenius, K., Lemke, E. A., Grønborg, M., Riedel, D., Urlaub, H., Schenck, S., Brügger, B., Ringler, P., Müller, S. A., Rammner, B., Gräter, F., Hub, J. S., De Groot, B. L., Mieskes, G., Moriyama, Y., Klingauf, J., Grubmüller, H., Heuser, J., Wieland, F. and Jahn, R. (2006). Molecular anatomy of a trafficking organelle. Cell 127, 831-846. 
Tarantino, N., Tinevez, J.-Y., Crowell, E. F., Boisson, B., Henriques, R., Mhlanga, M., Agou, F., Israël, A. and Laplantine, E. (2014). TNF and IL-1 exhibit distinct ubiquitin requirements for inducing NEMO-IKK supramolecular structures. The Journal of Cell Biology 204, 231-245.

Tareste, D., Shen, J., Melia, T. J. and Rothman, J. E. (2008). SNAREpin/Munc18 promotes adhesion and fusion of large vesicles to giant membranes. Proceedings of the National Academy of Sciences 105, 2380-2385.

Tartof, K. D. and Hobbs, C. A. (1987). Improved media for growing plasmid and cosmid clones. Bethesda Res. Lab. Focus 9.

Vacquier, V. D. (1975). The isolation of intact cortical granules from sea urchin eggs: Calcium ions trigger granule discharge. Developmental Biology 43, 62-74.

Vamparys, L., Gautier, R., Vanni, S., Bennett, W. D., Tieleman, D. P., Antonny, B., Etchebest, C. and Fuchs, P. F. (2013). Conical Lipids in Flat Bilayers Induce Packing Defects Similar to that Induced by Positive Curvature. Biophysical Journal $104,585-593$.

van den Bogaart, G., Holt, M. G., Bunt, G., Riedel, D., Wouters, F. S. and Jahn, R. (2010). One SNARE complex is sufficient for membrane fusion. Nat Struct Mol Biol $17,358-364$.

van den Bogaart, G., Meyenberg, K., Risselada, H. J., Amin, H., Willig, K. I., Hubrich, B. E., Dier, M., Hell, S. W., Grubmüller, H., Diederichsen, U. and Jahn, R. (2011). Membrane protein sequestering by ionic protein-lipid interactions. Nature $479,552-$ 555.

Varoqueaux, F., Sigler, A., Rhee, J.-S., Brose, N., Enk, C., Reim, K. and Rosenmund, C. (2002). Total arrest of spontaneous and evoked synaptic transmission but normal synaptogenesis in the absence of Munc13-mediated vesicle priming. Proceedings of the National Academy of Sciences of the United States of America 99, 9037-9042.

Verhage, M., Maia, A. S., Plomp, J. J., Brussaard, A. B., Heeroma, J. H., Vermeer, H., Toonen, R. F., Hammer, R. E., van den Berg, T. K., Missler, M., Geuze, H. J. and Südhof, T. C. (2000). Synaptic assembly of the brain in the absence of neurotransmitter secretion. Science (New York, N.Y.) 287, 864-869.

Verhage, M. and Sørensen, J. B. (2008). Vesicle Docking in Regulated Exocytosis. Traffic 9, 1414-1424. 
Voets, T. (2000). Dissection of Three $\mathrm{Ca}^{2+}$-Dependent Steps Leading to Secretion in Chromaffin Cells from Mouse Adrenal Slices. Neuron 28, 537-545.

Wagner, M. L. and Tamm, L. K. (2000). Tethered polymer-supported planar lipid bilayers for reconstitution of integral membrane proteins: silane-polyethyleneglycollipid as a cushion and covalent linker. Biophysical journal 79, 1400-1414.

Wagner, M. L. and Tamm, L. K. (2001). Reconstituted syntaxin1a/SNAP25 interacts with negatively charged lipids as measured by lateral diffusion in planar supported bilayers. Biophysical journal 81, 266-275.

Warner, J. M., Karatekin, E. and O'Shaughnessy, B. (2009). Model of SNAREmediated membrane adhesion kinetics. PloS one 4, e6375.

Weber, T., Zemelman, B. V., McNew, J. A., Westermann, B., Gmachl, M., Parlati, F., Söllner, T. H. and Rothman, J. E. (1998). SNAREpins: minimal machinery for membrane fusion. Cell 92, 759-772.

Wegrzyn, J. L., Bark, S. J., Funkelstein, L., Mosier, C., Yap, A., Kazemi-Esfarjani, P., Spada, A. R. L., Sigurdson, C., O'Connor, D. T. and Hook, V. (2010). Proteomics of Dense Core Secretory Vesicles Reveal Distinct Protein Categories for Secretion of Neuroeffectors for Cell-Cell Communication. Journal of Proteome Research 9, $5002-5024$.

Wiederhold, K. and Fasshauer, D. (2009). Is assembly of the SNARE complex enough to fuel membrane fusion? The Journal of biological chemistry 284, 13143-13152.

Wiederhold, K., Kloepper, T. H., Walter, A. M., Stein, A., Kienle, N., Sørensen, J. B. and Fasshauer, D. (2010). A Coiled Coil Trigger Site Is Essential for Rapid Binding of Synaptobrevin to the SNARE Acceptor Complex. Journal of Biological Chemistry 285, 21549-21559.

Wiley, D. C. and Skehel, a. J. J. (1987). The Structure and Function of the Hemagglutinin Membrane Glycoprotein of Influenza Virus. Annual Review of Biochemistry $56,365-394$.

Wilschut, J., Duzgunes, N., Fraley, R. and Papahadjopoulos, D. (1980). Studies on the mechanism of membrane fusion: kinetics of calcium ion induced fusion of phosphatidylserine vesicles followed by a new assay for mixing of aqueous vesicle contents. Biochemistry 19, 6011-6021. 
Winter, U., Chen, X. and Fasshauer, D. (2009). A conserved membrane attachment site in alpha-SNAP facilitates N-ethylmaleimide-sensitive factor (NSF)-driven SNARE complex disassembly. The Journal of biological chemistry 284, 31817-31826.

Witkowska, A. and Jahn, R. (2016). SNARE-mediated fusion of liposomes and chromaffin granules with giant unilamellar vesicles with millisecond kinetics. Submitted.

Wollert, T., Wunder, C., Lippincott-Schwartz, J. and Hurley, J. H. (2009). Membrane scission by the ESCRT-III complex. Nature 458, 172-177.

Xu, T., Binz, T., Niemann, H. and Neher, E. (1998). Multiple kinetic components of exocytosis distinguished by neurotoxin sensitivity. Nature Neuroscience 1, 192-200.

Xu, T., Rammner, B., Margittai, M., Artalejo, A. R., Neher, E. and Jahn, R. (1999). Inhibition of SNARE Complex Assembly Differentially Affects Kinetic Components of Exocytosis. Cell 99, 713-722.

Xue, M., Craig, T. K., Xu, J., Chao, H.-T., Rizo, J. and Rosenmund, C. (2010). Binding of the complexin N terminus to the SNARE complex potentiates synapticvesicle fusogenicity. Nature Structural \& Molecular Biology 17, 568-575.

Yang, H.-J., Sugiura, Y., Ikegami, K., Konishi, Y. and Setou, M. (2012). Axonal Gradient of Arachidonic Acid-containing Phosphatidylcholine and Its Dependence on Actin Dynamics. Journal of Biological Chemistry 287, 5290-5300.

Yang, X., Kaeser-Woo, Y. J., Pang, Z. P., Xu, W. and Südhof, T. C. (2010). Complexin Clamps Asynchronous Release by Blocking a Secondary $\mathrm{Ca}^{2+}$-Sensor via its Accessory $\alpha$-Helix. Neuron 68, 907-920.

Yavuz, H. (2015). In vitro investigation of trans SNARE complexes arrested between artificial membranes. PhD thesis, Göttingen Graduate School for Neurosciences, Biophysics, and Molecular Biosciences.

Yoon, T.-Y., Okumus, B., Zhang, F., Shin, Y.-K. and Ha, T. (2006). Multiple intermediates in SNARE-induced membrane fusion. Proceedings of the National Academy of Sciences 103, 19731-19736.

Yuan, T., Lu, J., Zhang, J., Zhang, Y. and Chen, L. (2015). Spatiotemporal Detection and Analysis of Exocytosis Reveal Fusion "Hotspots" Organized by the Cytoskeleton in Endocrine Cells. Biophysical Journal 108, 251-260. 
Zenisek, D., Steyer, J. A. and Almers, W. (2000). Transport, capture and exocytosis of single synaptic vesicles at active zones. Nature 406, 849-854.

Zenisek, D., Steyer, J. A., Feldman, M. E. and Almers, W. (2002). A Membrane Marker Leaves Synaptic Vesicles in Milliseconds after Exocytosis in Retinal Bipolar Cells. Neuron 35, 1085-1097.

Zhang, Z. and Jackson, M. B. (2008). Temperature Dependence of Fusion Kinetics and Fusion Pores in $\mathrm{Ca}^{2+}$-triggered Exocytosis from PC12 Cells. The Journal of General Physiology 131, 117-124.

Zhou, Q., Lai, Y., Bacaj, T., Zhao, M., Lyubimov, A. Y., Uervirojnangkoorn, M., Zeldin, O. B., Brewster, A. S., Sauter, N. K., Cohen, A. E., Soltis, S. M., Alonso-Mori, R., Chollet, M., Lemke, H. T., Pfuetzner, R. A., Choi, U. B., Weis, W. I., Diao, J., Südhof, T. C. and Brunger, A. T. (2015). Architecture of the synaptotagmin-SNARE machinery for neuronal exocytosis. Nature 525, 62-67. 


\section{Appendix}

\section{Tables}

Table A.1. Reagents. List of specific reagents used in this study.

\begin{tabular}{ccc}
\hline Reagent & Catalogue Number & Company \\
\hline \hline Oregon Green 488 Iodoacetamide & O6010 & ThermoFisher Scientific \\
\hline Texas Red C2 Maleimide & T6008 & ThermoFisher Scientific \\
\hline Hellmanex II & RV 800237 & Hellma Analytics \\
\hline $\begin{array}{c}\text { Pierce Bovine Serum Albumin, } \\
\text { Biotinylated }\end{array}$ & 29130 & ThermoFisher Scientific \\
\hline NeutrAvidin Protein & 31000 & ThermoFisher Scientific \\
\hline Calcein disodium salt & 21030 & Sigma-Aldrich \\
\hline Sulforhodamine B sodium salt & S9012 & Sigma-Aldrich \\
\hline Bio-Beads® SM-2 Adsorbent & 1523920 & BioRad \\
\hline
\end{tabular}


Table A.2. List of detergents tested for $\Delta \mathrm{N}$ complex reconstitution into preformed GUVs. Critical micelle concentration (CMC) values are taken from manufacturer's website (all detergents, except for OG, are from Anatrace). For experimental details see Section 3.1.1.

\begin{tabular}{|c|c|c|}
\hline Detergent full name & Abbreviation & $\mathrm{CMC}(\mathrm{mM})$ \\
\hline n-Dodecyl- $\beta$-Maltoside & $\mathrm{DDM}$ & 0.2 \\
\hline n-Nonyl- $\beta$-D-Thiomaltopyranoside & NTM & 3.2 \\
\hline 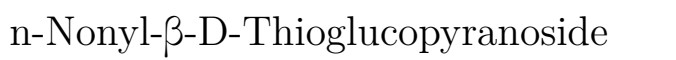 & NTG & 2.9 \\
\hline n-Heptyl- $\beta$-D-Glucopyranoside & $\mathrm{HG}$ & 70 \\
\hline n-Nonyl- $\beta$-D-Glucopyranoside & $\mathrm{NG}$ & 7 \\
\hline n-Nonyl- $\beta$-Maltoside & $\mathrm{NM}$ & 6 \\
\hline n-Decyl- $\beta$-D-Glucopyranoside & DG & 2.2 \\
\hline n-Dodecyl- $\beta$-D-Thiomaltopyranoside & DOTM & 0.05 \\
\hline Octyl $\beta$-D-Glucopyranoside & $\mathrm{OG}$ & $17^{a}$ \\
\hline
\end{tabular}

${ }^{a}$ Rigaud and Lévy, 2003 
Table A.3. Phospholipids and lipophilic tracers. Lipids were ordered in a powdered form. Stock solutions were prepared in 2:1 chloroform:methanol and stored at $-20{ }^{\circ} \mathrm{C}$ in sealed glass vials purged with argon. Lipophilic tracers (DiO and $\mathrm{DiD}$ ) were prepared in DMSO (dimethyl sulfoxide).

\begin{tabular}{|c|c|c|c|}
\hline \multicolumn{2}{|c|}{ Phospholipid or lipophilic tracer } & \multirow{2}{*}{$\begin{array}{c}\begin{array}{c}\text { Catalogue } \\
\text { Number }\end{array} \\
840053 \mathrm{P}\end{array}$} & \multirow[t]{2}{*}{ Company } \\
\hline $\mathrm{PC}$ & $\begin{array}{c}\text { L- } \alpha \text {-phosphatidylcholine (Brain, } \\
\text { Porcine) }\end{array}$ & & \\
\hline $\mathrm{PE}$ & $\begin{array}{c}\text { L- } \alpha \text {-phosphatidylethanolamine } \\
\text { (Brain, Porcine) }\end{array}$ & $840022 \mathrm{P}$ & \multirow{6}{*}{ Avanti Polar Lipids } \\
\hline PS & $\begin{array}{l}\text { L- } \alpha \text {-phosphatidylserine (Brain, } \\
\text { Porcine) }\end{array}$ & $840032 \mathrm{P}$ & \\
\hline chol & cholesterol (ovine wool) & $700000 \mathrm{P}$ & \\
\hline biot-PE & $\begin{array}{c}\text { 1,2-dioleoyl-sn-glycero-3- } \\
\text { phosphoethanolamine-N-(cap } \\
\text { biotinyl) }\end{array}$ & $870273 \mathrm{P}$ & \\
\hline NBD-PE & $\begin{array}{c}\text { 1,2-dioleoyl-sn-glycero-3- } \\
\text { phosphoethanolamine-N-(7-nitro-2- } \\
\text { 1,3-benzoxadiazol-4-yl) }\end{array}$ & $810145 \mathrm{P}$ & \\
\hline Rho-PE & $\begin{array}{l}\text { 1,2-dioleoyl-sn-glycero-3- } \\
\text { phosphoethanolamine-N-(lissamine } \\
\text { rhodamine B sulfonyl) }\end{array}$ & $810150 \mathrm{P}$ & \\
\hline TR-PE & $\begin{array}{c}\text { Texas Red® 1,2-Dihexadecanoyl-sn- } \\
\text { Glycero-3-Phosphoethanolamine, } \\
\text { Triethylammonium Salt }\end{array}$ & $\mathrm{T} 1395 \mathrm{MP}$ & \multirow{5}{*}{ ThermoFisher Scientific } \\
\hline OG488-PE & $\begin{array}{c}\text { Oregon Green }{ }^{\circledR} 488 \\
\text { 1,2-Dihexadecanoyl-sn-Glycero-3- } \\
\text { Phosphoethanolamine }\end{array}$ & O12650 & \\
\hline $\mathrm{DiO}$ & $\begin{array}{c}\text { 3,3'-Dioctadecyl-5,5'-Di(4- } \\
\text { Sulfophenyl)Oxacarbocyanine, } \\
\text { Sodium Salt }\end{array}$ & D7778 & \\
\hline $\mathrm{DiD}$ & $\begin{array}{c}\text { 1,1'-Dioctadecyl-3,3,3',3'- } \\
\text { Tetramethylindodicarbocyanine- } \\
\text { 5,5'-Disulfonic } \\
\text { Acid }\end{array}$ & D12730 & \\
\hline TMA-DPH & $\begin{array}{c}\text { 1-(4-Trimethylammoniumphenyl)- } \\
\text { 6-Phenyl-1,3,5-Hexatriene } \\
\text { p-Toluenesulfonate }\end{array}$ & T204 & \\
\hline
\end{tabular}


Table A.4. Equipment. List of specific equipment and instruments used in this study.

\begin{tabular}{|c|c|c|}
\hline Equipment & Characteristics/Model & Manufacturer \\
\hline $\begin{array}{c}\text { Liquid } \\
\text { chromatography } \\
\text { system }\end{array}$ & $\begin{array}{c}\text { ÄKTA Protein Purification } \\
\text { System }\end{array}$ & GE Healthcare \\
\hline Anion exchanger & Mono Q 10/100 and 5/50 GL & \\
\hline Cation exchanger & Mono S 10/100 GL & \\
\hline ITO-coated glasses & 15-30 Ohm & Diamond Coatings \\
\hline Function generator & PCGU1000 & Velleman \\
\hline Fluorolog 3 & FL322 & Jobin Yvon \\
\hline Quartz cuvette & $28 \mathrm{~F}-\mathrm{Q}-10$ & Starna GmbH \\
\hline Spectrophotometer & NanoDrop 1000 & Thermo Scientific \\
\hline Microplate reader & Genios Pro & TECAN \\
\hline Rotary evaporator & Rotavapor R-124 & BÜCHI \\
\hline Liposome extruder & Mini Extruder & Avanti Polar Lipids \\
\hline Sonifier & Sonifier 450 & Branson \\
\hline Glass coverslips & $\begin{array}{c}18 \mathrm{~mm} \varnothing, \text { thickness No. } 1.5 \\
(0.170 \pm 0.005 \mathrm{~mm})\end{array}$ & $\begin{array}{l}\text { Paul Marienfeld GmbH \& } \\
\text { Co.KG }\end{array}$ \\
\hline $\begin{array}{l}\text { Imaging chamber } \\
\text { and stage adaptor }\end{array}$ & $\begin{array}{l}\text { Chamber holding coverslips } \\
\text { and matching microscope } \\
\text { stage adaptors for Zeiss and } \\
\text { Leica microscopes }\end{array}$ & $\begin{array}{l}\text { in-house workshop, Max } \\
\text { Planck Institute for Bio- } \\
\text { physical Chemistry }\end{array}$ \\
\hline Widefield microscope & Axiovert $200 \mathrm{M}$ & Carl Zeiss \\
\hline $\begin{array}{l}\text { Scanning Confocal } \\
\text { Microscope }\end{array}$ & LSM 780, AxioObserver & Carl Zeiss \\
\hline $\begin{array}{l}\text { Scanning Confocal } \\
\text { Microscope }\end{array}$ & TCS SP8 & Leica Microsystems GmbH \\
\hline iSCAT microscope & $\begin{array}{l}\text { home-build iSCAT } \\
\text { microscopy setup }\end{array}$ & $\begin{array}{l}\text { Susann Spindler and } \\
\text { Prof. Vahid Sandoghdar } \\
\text { (Max Planck Institute } \\
\text { for the Science of Light, } \\
\text { Erlangen, Germany) }\end{array}$ \\
\hline
\end{tabular}


Table A.5. Miscellaneous products. List of miscellaneous products used in this study.

\begin{tabular}{ccc}
\hline Reagent & $\begin{array}{c}\text { Catalogue } \\
\text { Number }\end{array}$ & Company \\
\hline \hline $\begin{array}{c}\text { Sephadex G-50 Superfine } \\
\text { Disposable PD-10 Desalting Column, with } \\
\text { Sephadex G-25 resin }\end{array}$ & G5050 & Sigma-Aldrich \\
\hline $\begin{array}{c}\text { Slide-A-Lyzer MINI Dialysis Device, 2K } \\
\text { MWCO, 0.1 ml }\end{array}$ & 69580 & GE Healthcare \\
\hline $\begin{array}{c}\text { Slide-A-Lyzer Dialysis Cassettes, 2K } \\
\text { MWCO, 0.5 ml }\end{array}$ & 66205 & ThermoFisher Scientific \\
\hline $\begin{array}{c}\text { Pierce 660nm Protein Assay Kit } \\
\text { Copper tape with conductive adhesive }\end{array}$ & 22662 & ThermoFisher Scientific \\
\hline $\begin{array}{c}\text { Nuclepore Polycarbonate Hydrophilic } \\
\text { Membranes, circles, 19 mm } \varnothing, \text { 0.1 and } \\
\text { 0.4 } 1181\end{array}$ & 800309 & 3M pore size \\
\hline
\end{tabular}


Table A.6. Solutions. Composition of specific buffers used in this study.

\begin{tabular}{|c|c|c|}
\hline Solution & Components & Concentration \\
\hline \multirow{3}{*}{ resuspension buffer } & $\operatorname{HEPES}(\mathrm{pH} 7.4)$ & $20 \mathrm{mM}$ \\
\hline & $\mathrm{NaCl}$ & $500 \mathrm{mM}$ \\
\hline & imidazole & $8 \mathrm{mM}$ \\
\hline \multirow{4}{*}{ extraction buffer } & HEPES (pH 7.4) & $20 \mathrm{mM}$ \\
\hline & $\mathrm{NaCl}$ & $500 \mathrm{mM}$ \\
\hline & imidazole & $8 \mathrm{mM}$ \\
\hline & sodium cholate & $10 \% \mathrm{w} / \mathrm{v}$ \\
\hline \multirow{4}{*}{ wash buffer } & HEPES (pH 7.4) & $20 \mathrm{mM}$ \\
\hline & $\mathrm{NaCl}$ & $500 \mathrm{mM}$ \\
\hline & imidazole & $20 \mathrm{mM}$ \\
\hline & sodium cholate $^{a}$ & $1 \% \mathrm{w} / \mathrm{v}$ \\
\hline \multirow{4}{*}{ elution buffer } & HEPES (pH 7.4) & $20 \mathrm{mM}$ \\
\hline & $\mathrm{NaCl}$ & $500 \mathrm{mM}$ \\
\hline & imidazole & $400 \mathrm{mM}$ \\
\hline & $\mathrm{CHAPS} / \mathrm{OG}^{a}$ & $1 \% \mathrm{w} / \mathrm{v}$ \\
\hline \multirow{5}{*}{ dialysis buffer } & HEPES (pH 7.4) & $20 \mathrm{mM}$ \\
\hline & $\mathrm{NaCl}$ & depending on protein \\
\hline & EDTA & $1 \mathrm{mM}$ \\
\hline & DTT & $1 \mathrm{mM}$ \\
\hline & $\mathrm{CHAPS} / \mathrm{OG}^{a}$ & $1 \% \mathrm{w} / \mathrm{v}$ \\
\hline \multirow{3}{*}{ Coomasie staining } & Coomassie Blue R-250 & $0.25 \% \mathrm{w} / \mathrm{v}$ \\
\hline & methanol & $46 \% \mathrm{v} / \mathrm{v}$ \\
\hline & acetic acid & $10 \% \mathrm{v} / \mathrm{v}$ \\
\hline \multirow{2}{*}{ Commassie destaining solution 1} & ethanol & $50 \% \mathrm{v} / \mathrm{v}$ \\
\hline & acetic acid & $10 \% \mathrm{v} / \mathrm{v}$ \\
\hline \multirow{2}{*}{ Commassie destaining solution 2} & ethanol & $10 \% \mathrm{v} / \mathrm{v}$ \\
\hline & acetic acid & $5 \% \mathrm{v} / \mathrm{v}$ \\
\hline \multirow{4}{*}{ liposome buffer } & HEPES (pH 7.4) & $20 \mathrm{mM}$ \\
\hline & $\mathrm{KCl}$ & $150 \mathrm{mM}$ \\
\hline & $\mathrm{EDTA}^{b}$ & $1 \mathrm{mM}$ \\
\hline & $\mathrm{DTT}^{b}$ & $1 \mathrm{mM}$ \\
\hline \multirow{5}{*}{ disassembly buffer ${ }^{c}$} & HEPES (pH 7.4) & $50 \mathrm{mM}$ \\
\hline & potassium glutamate & $120 \mathrm{mM}$ \\
\hline & potassium acetate & $20 \mathrm{mM}$ \\
\hline & ATP & $2 \mathrm{mM}$ \\
\hline & $\mathrm{MgCl}_{2}$ & $5 \mathrm{mM}$ \\
\hline
\end{tabular}

\footnotetext{
${ }^{a}$ supplemented only in case of proteins containing transmembrane domain ${ }^{b}$ optional

${ }^{c}$ Winter et al. 2009
} 
Table A.7. Microscope configurations. Specifications and basic settings of microscopes used in this study.

\begin{tabular}{|c|c|c|c|c|c|}
\hline & Objective & $\begin{array}{l}\text { Illumination/ } \\
\text { Excitation }\end{array}$ & Emission & Software & Other \\
\hline $\begin{array}{l}\text { Zeiss } \\
\text { Axiovert } \\
200 \mathrm{M}\end{array}$ & $\begin{array}{l}\text { Plan-Apochromat } \\
100 \times / 1.40 \text { Oil } \\
\text { Plan-Apochromat } \\
63 \times / 1.40 \text { Oil DIC }\end{array}$ & $\begin{array}{l}\text { Xenon-short-arc } \\
\text { lamp XBO } 75\end{array}$ & $\begin{array}{l}\text { AxioCam } \\
\text { MR3 }\end{array}$ & AxioVision & $\begin{array}{l}\text { Filter Set } 46 \\
(000000-1196-681): \text { BP } \\
500 / 20, \text { FT 515, BP 535/30 } \\
\text { Filter Set } 43 \\
(000000-1114-101): \text { BP } \\
545 / 25, \text { FT 570, BP 605/70 } \\
\text { Filter Set 50 } \\
\text { (488050-9901-000): BP } \\
640 / 30, \text { FT 660, BP 690/50 }\end{array}$ \\
\hline $\begin{array}{l}\text { Zeiss } \\
\text { LSM 780, } \\
\text { AxioObserver }\end{array}$ & $\begin{array}{l}\text { LCI Plan-Neofluar } \\
63 \times / 1.3 \text { Imm Korr } \\
\text { DIC M27 }\end{array}$ & $\begin{array}{l}\text { Lasers: Argon, } \\
\text { DPSS561, } \\
\text { HeNe594, HeNe633 }\end{array}$ & $\mathrm{PMT}$ & Zen 2010 & - \\
\hline $\begin{array}{l}\text { Leica } \\
\text { TCS SP8 }\end{array}$ & $\begin{array}{l}\text { HC PL APO CS2 } \\
63 \times / 1.40 \text { OIL }\end{array}$ & $\begin{array}{l}\text { Lasers: Argon, } \\
\text { DPSS 561, } \\
\text { HeNe633 }\end{array}$ & $\begin{array}{l}\text { PMT } \\
\text { HyD }\end{array}$ & LAS X & - \\
\hline iSCAT & $\begin{array}{l}\text { UPLSAPO } \\
100 \times / 1.4 \\
\text { Olympus }\end{array}$ & $\begin{array}{l}\text { LED lamp, } \\
\text { Laser: } 532 \mathrm{~nm} \\
\text { continuous-wave } \\
\text { laser }\end{array}$ & $\begin{array}{l}\text { CMOS } \\
\text { camera }\end{array}$ & custom & $\begin{array}{l}\text { home-build iSCAT microscopy } \\
\text { setup by Susann Spindler and } \\
\text { Prof. Vahid Sandoghdar (Max } \\
\text { Planck Institute for } \\
\text { the Science of Light in } \\
\text { Erlangen, Germany), similar } \\
\text { to Hsieh et al. (2014) }\end{array}$ \\
\hline
\end{tabular}




\section{Scripts}

\section{Script 1. ImageJ macro for analysis of GUV membrane fluorescence in-} tensity. This macro automatically localizes a GUV on an image and linearizes its membrane.

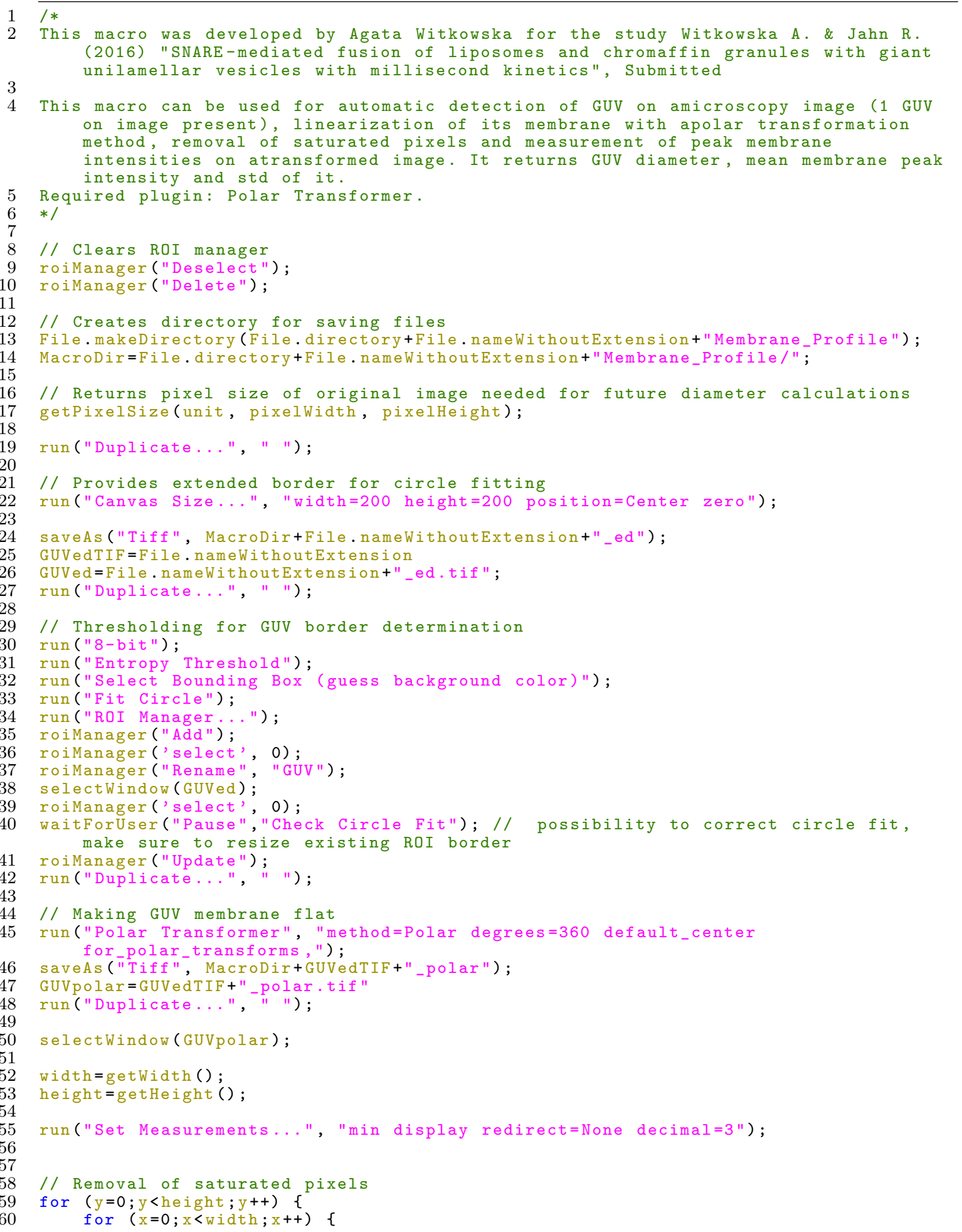




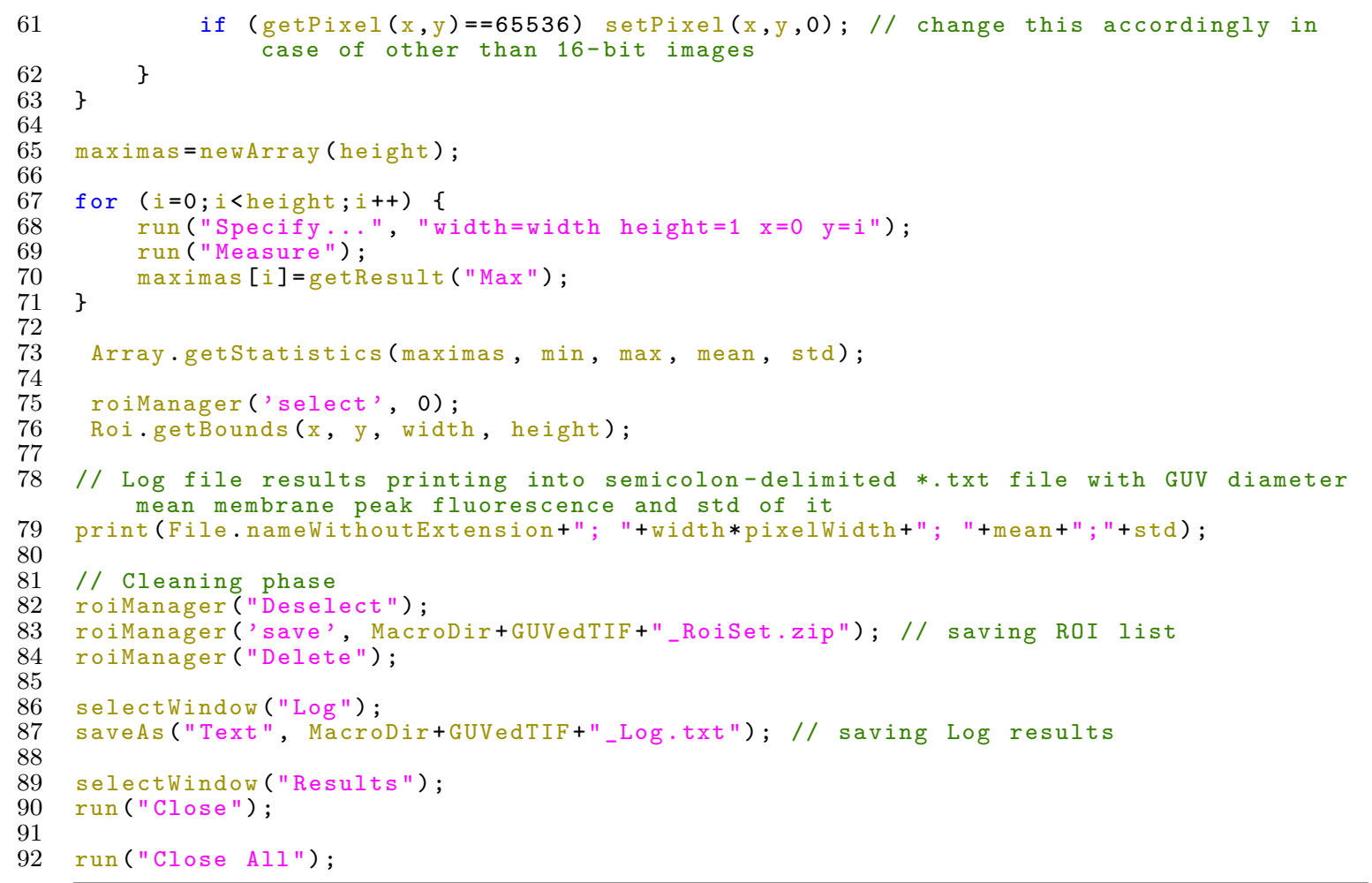


Script 2. ImageJ macro for analysis of FRAP data. This script extracts bleach settings and fluorescence intensities from FRAP image stacks from ZEN 2010 (Zeiss). Output data is formatted for analysis with Script 3 in GNU Octave that automatically strats at the end of this script.

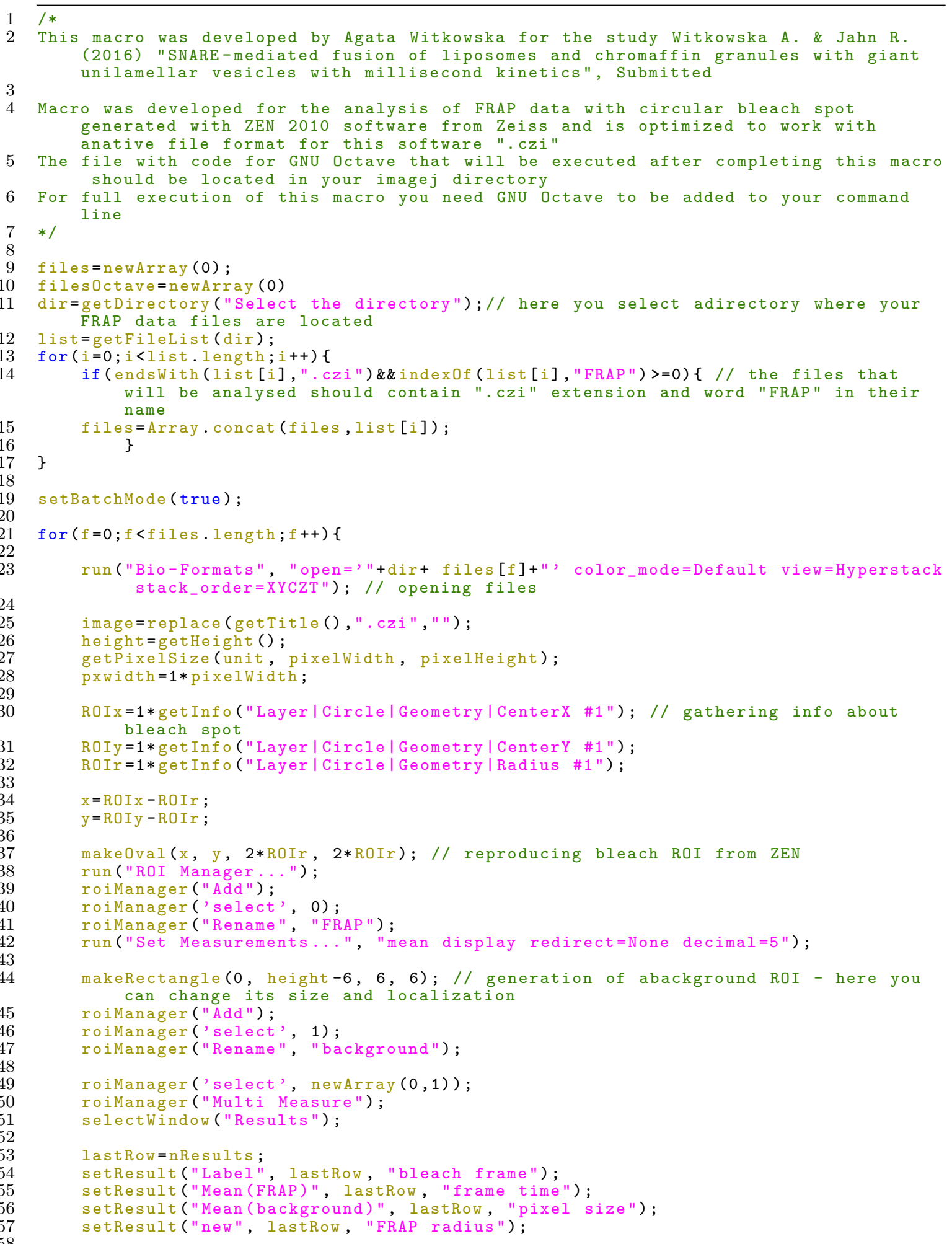




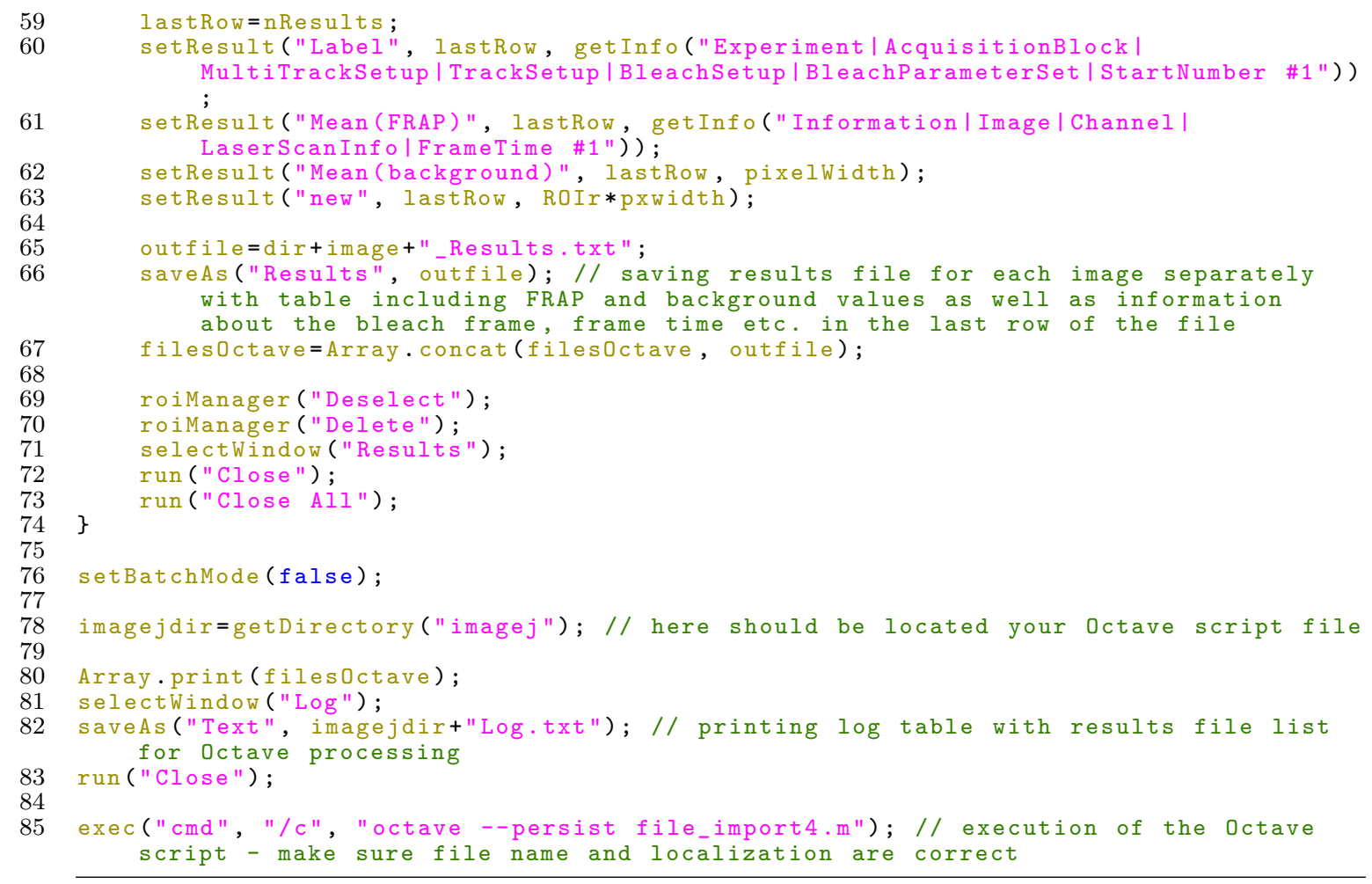


Script 3. GNU Octave script for analysis of FRAP data. This script was developed based on description by Miura (2012).

$\%\{$

This macro was developed by Agata Witkowska for the study Witkowska A. \& Jahn R. (2016) "SNARE-mediated fusion of liposomes and chromaffin granules with giant unilamellar vesicles with millisecond kinetics", Submitted

Script was developed for the analysis of FRAP data with circular bleach spot generated with ZEN 2010 software from Zeiss and is optimized to work with anative file format for this software ".czi" and is intended to use with accompanying Image J macro

6 It groups multiple FRAP traces into groups with the same bleach parameters and

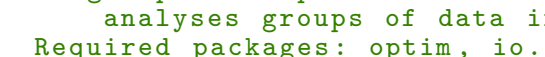

$8 \%\}$

clear all;

close all:

pkg load optim;

pkg load io;

\% Import of multiple files version

[DIR, NAME, EXT]=fileparts (mfilename("fullpathext")); \% directory, name and extension of this script file

filelist=textread (fullfile(DIR, 'Log.txt'), '\%s', 'Delimiter', ', ');

norm2sort $=$ struct

for $f=1:$ length ( $f$ ilelist)

file=fopen(filelist $\{f\}) ; \%$ open file with results

[DIRDATA, NAMEDATA, EXTDATA]=fileparts (filelist $\{f\}$ );

nlines=fskipl (file, Inf); \% count number of lines in file

frewind (file); $\%$ set the file position to the beginning

data=dlmread (file, '\t', [1 2 nlines-3 3]) ; \% load "Mean(FRAP) " and "Mean( background)" columns to data

frewind(file); \% set the file position to the beginning

params=dlmread (file, '\t', [nlines-1 1 nlines 4]); \% load "buffer frame", "frame time", "pixel size" and "FRAP radius" to params

params =num2cell (params); \% make separate cells in params

[pbf ft px fr]=deal (params $\{:\}) ; \%$ pbf - "prebleach frames"; ft - "frame time"; px - "pixel size"; fr - "FRAP radius"

clear params; \% remove params array

fclose (file); \% close file

\% Two-step normalisation accoriding to Miura, K. (2012) "Analysis of FRAP Curves " European Advanced Light Microscopy Network

tbleach $=p b f * t$

Ifrap_pre $=0$;

$\mathrm{n}=$ length (data)

data $=[$ data $\operatorname{zeros}(n, 2)]$

for $t=1: p b f$;

Ifrap_pre $+=(\operatorname{data}(t, 1)-\operatorname{data}(t, 2)) / \mathrm{pbf}$;

end

for $t=1: n$;

data $(t, 3)=(\operatorname{data}(t, 1)-\operatorname{data}(t, 2)) /$ Ifrap_pre; \% first normalisation norm 1

end

Ifrap_bleach $=$ data $\left(p_{b f}+1,3\right)$;

Ifrap pre norm $=$ mean $(\operatorname{data}(1: \operatorname{pbf}, 3))$;

for $t=1: n$;

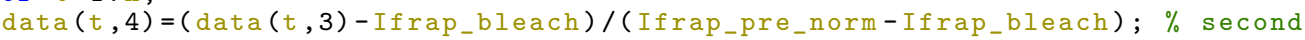
normalisation norm 2

end

\% Generation of structured array with experiments grouped according to pbf, ft, px, fr and no of timepoints

grname=strrep (["gr " num2str(pbf) " " num2str(ft) " " num2str(px) " " num2str

(fr) "- " num2str(length(data))], ".", "_"); \% group name

if (! isfield (norm2sort, grname))

norm2sort. (grname) params $=[p b f$ ft px fr]; \% group params pbf ft px fr

norm2sort. (grname). time=colon $(0, n-1) * f t ; \%$ group name time endif

if (isfield(norm2sort.(grname), "all"))

norm2sort. (grname). all=[norm2sort. (grname).all;transpose (data (:,4))]; \% adds norm2 to the array with all norm2 data from one group else 
norm2sort. (grname). all=transpose (data $(:, 4)) ; \%$ creates array with all norm2 data endif 


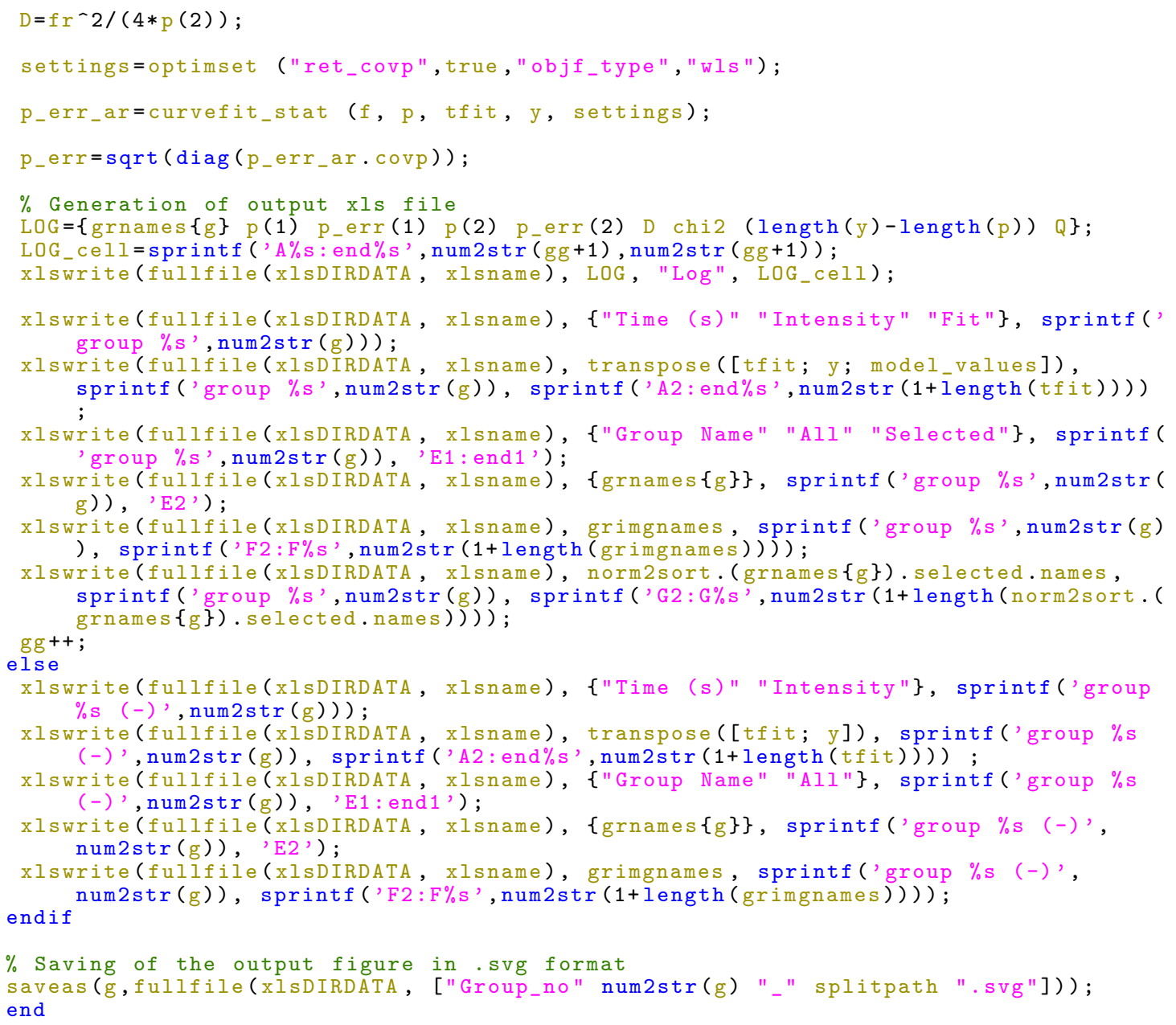




\section{List of Abbreviations}

$\begin{array}{ll}\text { AC } & \text { alternating current } \\ \text { ATP } & \text { adenosine triphosphate } \\ \text { CATCHR } & \text { complexes associated with tethering containing helical rods } \\ \text { CG } & \text { chromaffin granule } \\ \text { CHAPS } & \text { 3-[(3-Cholamidopropyl)dimethylammonio]-1-propanesulfonate } \\ \text { CMC } & \text { critical micelle concentration } \\ \text { CMOS } & \text { complementary metal-oxide-semiconductor } \\ \text { DMSO } & \text { dimethyl sulfoxide }\end{array}$

$\triangle \mathrm{N}$ complex $\quad$ SNARE acceptor complex consisting of plasma membrane SNAREs: syntaxin and SNAP-25, stabilized with a C-terminal fragment (4996) of synaptobrevin 2

DOL degree of labelling

DTT dithiothreitol

EDTA ethylenediaminetetraacetic acid

ESCRT endosomal sorting complexes required for transport

FRAP fluorescence recovery after photobleaching

FRET fluorescence (also Förster) resonance energy transfer

GLUT4 glucose transporter 4

GUV giant unilamellar vesicle

HEPES 4-(2-hydroxyethyl)-1-piperazineethanesulfonic acid

IPTG isopropyl $\beta$-D-1-thiogalactopyranoside

iSCAT interferometric scattering microscopy

ITO indium tin oxide 


\begin{tabular}{|c|c|}
\hline LB & lysogeny broth \\
\hline LUV & large unilamellar vesicle \\
\hline MSD & mean square displacement \\
\hline MWCO & molecular weight cut-off \\
\hline NSF & N-ethylmaleimide-sensitive factor \\
\hline OG & octyl $\beta$-D-glucopyranoside \\
\hline OG488 & Oregon Green 488 \\
\hline PIP2 & phosphatidylinositol 4,5-bisphosphate \\
\hline PIP3 & phosphatidylinositol $(3,4,5)$-trisphosphate \\
\hline PMSF & phenylmethanesulfonyl fluoride \\
\hline $\mathrm{SD}$ & standard deviation \\
\hline SDS & sodium dodecyl sulfate \\
\hline SEM & standard error of the mean \\
\hline SLB & supported lipid bilayer \\
\hline $\mathrm{SM}$ & Sec1/Munc18-like \\
\hline SNAP & Soluble NSF Attachment Protein \\
\hline SNARE & SNAP Receptor \\
\hline SUV & small unilamellar vesicle \\
\hline syb & synaptobrevin 2 \\
\hline TB & terrific broth \\
\hline TIRF & total internal reflection fluorescence \\
\hline TMD & transmembrane domain \\
\hline TR & Texas Red \\
\hline$V_{\mathrm{pp}}$ & peak-to-peak voltage \\
\hline$V_{\mathrm{RMS}}$ & root mean square voltage \\
\hline
\end{tabular}




\section{List of Figures}

1.1 Chemical neurotransmission principle . . . . . . . . . . . . 16

1.2 Neuronal SNARE complex structure . . . . . . . . . . . . . . . . . 19

1.3 The SNARE cycle . . . . . . . . . . . . . . . . . . 20

1.4 Hypothetical transition states in membrane fusion . . . . . . . . . . 23

1.5 Single vesicle fusion assays . . . . . . . . . . . . . . . . . . 27

1.6 Reconstruction of synaptic vesicle exocytosis in an in vitro assay . . . . 29

2.1 GUV preparation workflow . . . . . . . . . . . . . . 38

2.2 Electroformation chamber design . . . . . . . . . . . . . . . . . 39

2.3 Chromaffin granule purification procedure . . . . . . . . . . . . 40

2.4 Activity control for the purified chromaffin granules . . . . . . . . . . . 41

2.5 The iSCAT microscopy principle . . . . . . . . . . . . . . . . . 43

2.6 GUV immobilisation principle . . . . . . . . . . . . . . . . . . . 44

2.7 Optimisation of biotinylated lipid concentration needed for GUV immobilisation . . . . . . . . . . . . . . . . . . . 4 45

2.8 A GUV-based fusion assay design . . . . . . . . . . . . . . . . . . . . 46

2.9 Calibration curve for determination of protein reconstitution efficiency into GUVs . . . . . . . . . . . . . . . . . . . . . . . . . . . . . . 48

2.10 Diffusion coefficient determination of molecules incorporated into GUV membrane . . . . . . . . . . . . . . . . . . . 49

2.11 Analysis of vesicle diffusion on GUV surface by fluorescence microscopy 52

2.12 Diffusion coefficient determination from an iSCAT trajectory . . . . . 53

3.1 Image of a $\Delta \mathrm{N}-\mathrm{GUV} \ldots \ldots \ldots \ldots \ldots$ 
3.2 Comparison of $\Delta \mathrm{N}$ complex activity in GUVs prepared from SUVs or LUVs . . . . . . . . . . . . . . . . . . . . . 57

3.3 Comparison of $\Delta \mathrm{N}$ complex activity in GUVs prepared by electroformation in high or no salt buffer . . . . . . . . . . . . . . . . . . 58

3.4 Distribution of $\Delta \mathrm{N}-\mathrm{GUVs}$ diameter and lamellarity . . . . . . . . . 59

3.5 Determination of $\Delta \mathrm{N}$ complex concentration in a GUV membrane . . . 60

3.6 Determination of protein and lipid mobility in $\Delta \mathrm{N}-\mathrm{GUVs} \ldots \ldots 1$

3.7 Assembly and disassembly of SNARE complexes on GUV membrane (monitored in solution with fluorescence anisotropy) . . . . . . . . . 62

3.8 Assembly and disassembly of SNARE complexes on GUV membrane (monitored with microscopy) . . . . . . . . . . . . . . 63

3.9 Ternary SNARE complex formation on $\Delta$ N-GUV membrane . . . . . . 64

3.10 SNARE acceptor complex reconstituted into GUVs is able to induce lipid mixing between GUVs and syb-liposomes . . . . . . . . . . . . 65

3.11 SNARE acceptor complex reconstituted into GUVs is able to induce content mixing between GUVs and syb-liposomes . . . . . . . . . . . . 66

3.12 Single LUV docked at the $\Delta \mathrm{N}-\mathrm{GUV}$ membrane $\ldots \ldots \ldots$. . . . . . 67

3.13 Coverslip related artefacts of liposome attachment . . . . . . . . . . . 68

3.14 Single liposome fusion induced lipid mixing . . . . . . . . . . . . . . . 69

3.15 Single liposome fusion induced content mixing . . . . . . . . . . . . . 70

3.16 Single chromaffin granule fusing to a $\Delta \mathrm{N}-\mathrm{GUV} \ldots \ldots$. . . . . . . . . 71

3.17 Fast fusion kinetics of chromaffin granules . . . . . . . . . . . 72

3.18 Vesicle docking and undocking . . . . . . . . . . . . . . . 73

3.19 Reversible interactions of LUVs and GUVs . . . . . . . . . . . . . . 74

3.20 Docked vesicle mobility on the GUV surface . . . . . . . . . . . . . . . 74 
3.21 Imaging of $\Delta \mathrm{N}-\mathrm{GUVs}$ with iSCAT microscopy $\ldots \ldots \ldots$. . . . . . . 75

3.22 Tracking of LUVs docked on the on the GUV surface with the use of iSCAT microscopy . . . . . . . . . . . . . . . . 76

3.23 Docking mode occurrence depends on the SNARE zippering degree . . 77

3.24 Comparison of diffusion coefficients of syb-LUVs docked on $\Delta \mathrm{N}-\mathrm{GUVs}$ investigated by iSCAT . . . . . . . . . . . . . . . . 78 



\section{List of Tables}

2.1 Protein constructs . . . . . . . . . . . . . . . . . . . . 32

3.1 Parameters of various conditions tested during protocol optimisation for $\Delta \mathrm{N}-\mathrm{GUV}$ formation . . . . . . . . . . . . . . 56

A.1 Reagents . . . . . . . . . . . . . . . . . . . 119

A.2 List of detergents tested for $\Delta \mathrm{N}$ complex reconstitution into preformed GUVs . . . . . . . . . . . . . . . . . 120

A.3 Phospholipids and lipophilic tracers . . . . . . . . . . . . . . . . . 121

A.4 Equipment . . . . . . . . . . . . . . . . . . . . 122

A.5 Miscellaneous products . . . . . . . . . . . . . . . . 123

A.6 Solutions . . . . . . . . . . . . . . . . . . . . . . . . . . . 124

A.7 Microscope configurations . . . . . . . . . . . . . . . . . 125 



\section{List of Scripts}

1 ImageJ macro for analysis of GUV membrane fluorescence intensity . . 126

2 Image J macro for analysis of FRAP data . . . . . . . . . . . . . . 128

3 GNU Octave script for analysis of FRAP data . . . . . . . . . . . . 130 



\section{Curriculum Vitae}

\section{Personal information}

Name

Nationality

\section{Education}

2nd January 2013

Since October 2012

20th \& 22nd August 2012

Since October 2011

March 2011-January 2013

23rd February 2011

February-July 2010

October 2007-March 2011
Agata Witkowska

Polish

Master of Science and Engineer in Biotechnology

PhD student in Molecular Biology

Oral defence of a master thesis — "Study of the molecular basis of TNF-induced glucocorticoid resistance in MEF cells" and graduation at Warsaw University of Life Sciences with a master (MSc) degree, with a total grade $5.0=\mathrm{A}$ (excellent)

$\mathrm{PhD}$ student in the International Max Planck Research School for Molecular Biology (working in the Department of Neurobiology, Max Planck Institute for Biophysical Chemistry) under supervision of Prof. Dr. Reinhard Jahn

Master's Examinations in the Molecular Biology Program, passed with a total grade of $1.50=\mathrm{A}$ (excellent)

University of Göttingen, Germany, student of the International Max Planck Research School for Molecular Biology

Warsaw University of Life Sciences, Poland, MSc studies at Interfaculty Studies of Biotechnology

Oral defence of an engineer thesis — "Construction of PRA carrier state diagnostic test for canine breeds of Polish Greyhound, Briard and Miniature Schnauzer" and graduation at Warsaw University of Life Sciences with an engineer (BSc) degree, with a total grade $5.0=\mathrm{A}$ (excellent)

Ghent University, Belgium, Faculty of Sciences, studying as an Erasmus exchange biotechnology student

Warsaw University of Life Sciences, Poland, engineer studies at Interfaculty Studies of Biotechnology 


\section{Scholarships and \\ Awards}

September 2016

August 2016

August 2016

June/July 2014

January 2013-May 2014

October 2012-September 2015

October 2011-September 2012

October 2008-February 2011

February-June 2010

December 2008

\section{Publications}

\section{Research experience}

Since October 2012

Since October 2012
Awarded student talk during 13th Horizons in Molecular Biology (Göttingen, Germany)

Travel grant for the participation in the Gordon Research Conference "Synaptic Transmission"

Selected participant for the Gordon Research Conference "Synaptic Transmission" (Waterville Valley, USA)

Nomination for participation in the 64th Lindau Nobel Laureate Meeting (Physiology or Medicine) by the Nobel Laureate Prof. Dr. Erwin Neher

Mentee in the Dorothea Schlözer Mentoring Programme

Max Planck Society grant for PhD students

DAAD Study Scholarship for Graduates of All Disciplines

Warsaw University of Life Sciences, scholarship for scientific achievements

Scholarship from Erasmus Programme

Warsaw University of Life Sciences, award for the best posters on University Scientific Clubs Contest, for the poster "The use of tissue cultures in organisms" (co-author Julia Starońska)

Khuong, T. M., Habets, R. L., Kuenen, S., Witkowska, A., Kasprowicz, J., Swerts, J., Jahn, R., van den Bogaart, G. and Verstreken, P. (2013). Synaptic PI(3,4,5)P3 Is Required for Syntaxin1A Clustering and Neurotransmitter Release. Neuron 77, 1097-1108.

Witkowska, A. and Jahn, R. (2016). SNARE-mediated fusion of liposomes and chromaffin granules with giant unilamellar vesicles with millisecond kinetics. Submitted.

Max Planck Institute for Biophysical Chemistry, Department of Neurobiology, PhD project "Regulation of presynaptic proteinprotein interactions" - doctoral supervisor: Prof. Dr. Reinhard Jahn, 2nd and 3rd advisors: Prof. Dr. Andreas Janshoff and Prof. Dr. Stefan Jakobs

Participation in a Program Project Grant with research groups of Prof. Lucas Tamm and Prof. David Cafiso, funded by the National Institutes of Health 
Since October 2012

January-June 2012

October 2010-February 2011

February-July 2010

August 2009

April-June 2009

September 2008

\section{Conferences}

September 2016
Participation in a SFB803 project "Functionality controlled by organization in and between membranes" funded by the German Research Foundation

Molecular Biology Program, three 2-months research projects (lab rotations):

- "Confocal imaging of hair cell synaptic function", InnerEarLab, University Medical Center Göttingen, supervisor: Prof. Dr. Tobias Moser

- "The role of PIP2 and PIP3 in neuronal exocytosis", Department of Neurobiology, Max Planck Institute for Biophysical Chemistry, supervisor: Prof. Dr. Reinhard Jahn and Dr. Geert van den Bogaart

- "The role of PIP2 and PIP3 in myelin membrane growth", Laboratory of Cellular Neuroscience, Max Planck Institute of Experimental Medicine, supervisor: Prof. Dr. Mikael Simons

Warsaw University of Life Sciences, Faculty of Animal Science, Department of Genetics and Animal Breeding, research project in the Molecular Genetics Unit - "Construction of PRA carrier state diagnostic test for canine breed of Polish Greyhound, Briard and Miniature Schnauzer", supervisor: Dr. Zuzanna Nowak

Flanders Institute for Biotechnology (VIB), Department for Molecular Biomedical Research, research project in the Molecular Mouse Genetics Unit — "Study of the molecular basis of TNF-induced glucocorticoid resistance in MEF cells", supervisor: Prof. Dr. Claude Libert

Polish Academy of Sciences, Institute of Medical Biology, Laboratory of Cellular Proteomics, one month training under the supervision of Prof. Dr. Czeslaw Cierniewski (participation in a research project about thymosin $\beta 4$ )

Warsaw University of Life Sciences, Faculty of Veterinary Medicine, Department of Bacteriology and Molecular Biology, cooperation with Dr. Agnieszka Salamaszynska (participation in a research project about Brachyspira)

Polish Academy of Sciences, Institute of Genetics and Animal Breeding (Department of Experimental Embryology, Department of Molecular Cytogenetics and Department of Molecular Biology), 2-week training

Participation in 13th Horizons in Molecular Biology (Göttingen, Germany), oral and poster presentation 
August 2016

May 2015

October 2014

September 2014

June/July 2014

September 2013

June 2013

May 2013

October 2012

October 2012

September 2011

April 2011

November 2010

November 2009

December 2008
Participation in Gordon Research Conference "Synaptic transmission" (Waterville Valley, USA), poster presentation

Participation in Neurizons 2015 Conference (Göttingen, Germany), poster presentation

Participation in an International Symposium "Macromolecular Complexes in biosynthetic transport" (Heidelberg, Germany), poster presentation

Participation in and organization of 11th Horizons in Molecular Biology (Göttingen, Germany)

Participation in the 64th Lindau Nobel Laureate Meeting Physiology or Medicine (Lindau, Germany)

Participation in and organization of 10th Horizons in Molecular Biology (Göttingen, Germany)

Introductory presentation for the panel discussion Experts on Campus: "When money matters... Information and discussion on funding strategies for young academics", University of Göttingen (Germany)

Participation in Neurizons 2013 Conference (Göttingen, Germany)

Participation in the Life Science Open Day 2012, Weizmann Institute of Science (Rehovot, Israel)

Participation in 9th Horizons in Molecular Biology (Göttingen, Germany), poster presentation: "PIP2 and PIP3 in neuronal exocytosis"

Participation in 8th Horizons in Molecular Biology (Göttingen, Germany)

Participation in Neuronus 2011 Conference, Jagiellonian University (Cracow, Poland)

IInd International Student Conference of Biotechnology, XIIth Seminar of Biotechnology Students in Poland (Wroclaw, Poland), oral presentation: "Study of the molecular basis of TNFinduced glucocorticoid resistance in MEF cells"

Ist International Student Conference of Biotechnology, XIth Seminar of Biotechnology Students in Poland (Poznan, Poland), poster presentation "Two faces of thymosin $\beta 4$ "

Warsaw University of Life Sciences, University Scientific Clubs Contest (Warsaw, Poland), poster presentation "The use of tissue cultures in organisms" (co-author Julia Starońska) - award for the best poster 
November 2008

\section{Teaching experience}

2014-2016

2013-2016

January-December 2009

\section{Academic community Service}

January-December 2014

January-December 2014

May 2013-April 2014

2013-2014

October 2011-August 2012

2010-2011

May 2009

April 2009

2009
Xth Seminar of Biotechnology Students in Poland (Gdansk, Poland), poster presentation "The use of tissue cultures in organisms" (co-author Julia Starońska)

Instructor for the $\mathrm{PhD}$ level method course "Reconstitution of Neuronal Exocytosis"

Tutor for the Master's level courses: "Protein Sorting and Processing", "Membrane Traffic", and "Biological Membranes"

Warsaw University of Life Sciences, preparing and leading workshops for high school students (lectures and experiments) organized by Science Festival School (that is called Centre for Innovative Bioscience Education since 2010)

Member of the project group in the frame of Georg-August University School of Science (GAUSS), working on the preparation of a questionnaire about studying conditions (addressed to $\sim 1,500$ students)

$\mathrm{PhD}$ Student Representative to the Göttingen Graduate School for Neurosciences, Biophysics, and Molecular Biosciences (GGNB) Executive Board (representative of $\sim 500$ students)

PhD Student Representative to the Program Committee of IMPRS for Molecular Biology

Organizer of the Horizons in Molecular Biology 2013 and 2014, International PhD Student Symposium

Master Student Representative to the Program Committee of IMPRS for Molecular Biology

Inventor, and chief organizer of the Ist and the IInd Contests For the Best Students' Scientific Projects (grants for realisation), Warsaw University of Life Sciences (this event still exists and is organized every year)

Coordinator of the stand of Warsaw University of Life Sciences Students' Scientific Club of Biotechnologists on Science Picnic of Polish Radio and Copernicus Science Centre (preparing shows and experiments)

Member of organizing committee during IInd workshops "DNA — the encyclopedia of life"

Head of the Didactic Department in the Students' University Council of the Warsaw University of Life Sciences (representative of $\sim 25,000$ students) 
2008 and 2010

2008-2009

$2008-2011$

\section{Languages}

Mother tongue

Other languages
Member of the Didactic Department in the Students' University Council of the Warsaw University of Life Sciences (representative of $\sim 25,000$ students)

Representative of Interfaculty Studies of Biotechnology to the Students' University Council of the Warsaw University of Life Sciences (representative of $\sim 250$ students)

Member of the Young European Biotech Network — YEBN

Member of the Academic Society of Biotechnology Students ASSB

Member of the Students' Scientific Club of Biotechnologists, Warsaw University of Life Sciences

\section{Polish}

English (professional proficiency)

German (advanced)

Spanish (lower intermediate)

Dutch (elementary) 